\title{
RELATIVE STANLEY-REISNER THEORY AND UPPER BOUND THEOREMS FOR MINKOWSKI SUMS
}

KARIM A. ADIPRASITO AND RAMAN SANYAL

\begin{abstract}
In this paper we settle two long-standing questions regarding the combinatorial complexity of Minkowski sums of polytopes: We give a tight upper bound for the number of faces of a Minkowski sum, including a characterization of the case of equality. We similarly give a (tight) upper bound theorem for mixed facets of Minkowski sums. This has a wide range of applications and generalizes the classical Upper Bound Theorems of McMullen and Stanley.

Our main observation is that within (relative) Stanley-Reisner theory, it is possible to encode topological as well as combinatorial/geometric restrictions in an algebraic setup. We illustrate the technology by providing several simplicial isoperimetric and reverse isoperimetric inequalities in addition to our treatment of Minkowski sums.
\end{abstract}

The Upper Bound Theorem (UBT) for polytopes is one of the cornerstones of discrete geometry. The UBT gives precise bounds on the 'combinatorial complexity' of a convex polytope $P$ as measured by the number of $k$-dimensional faces $f_{k}(P)$ in terms of its dimension and the number of vertices.

Upper Bound Theorem for polytopes. For a d-dimensional polytope $P$ on $n$ vertices and $0 \leq k<d$

$$
f_{k}(P) \leq f_{k}\left(\mathrm{Cyc}_{d}(n)\right)
$$

where $\mathrm{Cyc}_{d}(n)$ is a d-dimensional cyclic polytope on $n$ vertices. Moreover, equality holds for all $k$ whenever it holds for some $k_{0}, k_{0}+1 \geq\left\lfloor\frac{d}{2}\right\rfloor$.

Polytopes attaining the upper bound are called (simplicial) neighborly polytopes and are characterized by the fact that all non-faces are of dimension at least $\frac{d}{2}$. Cyclic polytopes are a particularly interesting class of neighborly polytopes whose combinatorial structure allows for an elementary and explicit calculation of $f_{k}\left(\mathrm{Cyc}_{d}(n)\right)$ in terms of $d$ and $n$; cf. [Zie95, Section 0]. The UBT was conjectured by Motzkin [Mot57] and proved by McMullen [McM70]. One of the salient features to note is that for given $d$ and $n$ there is a polytope that maximizes $f_{k}$ for all $k$ simultaneously - a priori, this is not to be expected.

In this paper we will address more general upper bound problems for polytopes and polytopal complexes. To state the main applications of the theory to be developed, recall that the Minkowski sum of polytopes $P, Q \subseteq \mathbb{R}^{d}$ is the polytope $P+Q=\{p+q: p \in P, q \in Q\}$. There is no understating the importance of Minkowski sums for modern mathematics. It is named after Hermann Minkowski [Min11], who inaugurated the rich theory of mixed volumes and geometric inequalities; see [Sch93]. Applications reach into algebraic geometry [Hov78, CLS11], geometry of numbers and packings, computational commutative algebra [GS93, Stu02], robot motion planning [Lat91], and game theory [MRTT53]. An important and practically relevant question is regarding the combinatorial complexity of $P+Q$ is in terms of $P$ and $Q$. More precisely the Upper Bound Problem for Minkowski sums (UBPM), raised (in print) by Gritzmann and Sturmfels [GS93], asks:

For given $k<d$ and $n_{1}, n_{2}, \ldots, n_{m}$, what is the maximal number of $k$-dimensional faces of the Minkowski sum $P_{1}+P_{2}+\cdots+P_{m}$ for polytopes $P_{1}, \ldots, P_{m} \subseteq \mathbb{R}^{d}$ with vertex numbers $f_{0}\left(P_{i}\right)=n_{i}$ for $i=1, \ldots, m$ ?

Date: January 19, 2021.

2010 Mathematics Subject Classification. 52B05, 13F55, 13H10, 05E45.

Key words and phrases. Minkowski sums, Upper Bound Theorems, relative simplicial complexes, Stanley-Reisner modules, Cayley complexes.

K. Adiprasito was supported by an EPDI/IPDE postdoctoral fellowship, a Minerva fellowship of the Max Planck Society, the DFG within the research training group "Methods for Discrete Structures" (GRK1408) and by the Romanian NASR, CNCS UEFISCDI, project PN-II-ID-PCE-2011-3-0533.

R. Sanyal was supported by European Research Council under the European Union's Seventh Framework Programme (FP7/20072013) / ERC grant agreement $n^{\circ} 247029$ and by the DFG-Collaborative Research Center, TRR 109 "Discretization in Geometry and Dynamics". 
A solution to the UBPM subsumes the UBT for $m=1$. For $m>1$, it is nontrivial even for $k=0$ : In [San09], a comparatively involved topological argument is employed to show that for $m \geq d$ the trivial upper bound of $n_{1} n_{2} \cdots n_{m}$ vertices can not be attained. On the constructive side, Fukuda and Weibel [FW07, FW10, Wei12] and Matschke-Pfeifle-Pilaud [MPP11] gave several constructions for Minkowski sums that potentially maximize the number of faces. In particular, the constructions maximize the number of low-dimensional faces and, in analogy to the classical situation, they will be called Minkowski neighborly families (see Sections 5 and 6). Weibel [Wei12] proved that the number of vertices of a Minkowski sum is maximized by Minkowski neighborly families. A recent breakthrough was achieved by Karavelas and Tzanaki [KT11] who resolved the UBPM for two summands and subsequently for three summands in collaboration with Konaxis [KKT15]. Both papers adapt McMullen's geometric approach via shellings but with a dramatic increase in the complexity of the arguments. In this paper we give a complete resolution of the UBPM including a characterization of the equality case using a simple algebraic setup.

Upper Bound Theorem for Minkowski sums (UBTM). For polytopes $P_{1}, \ldots, P_{m} \subseteq \mathbb{R}^{d}$ with $n_{1}, \ldots, n_{m}$ vertices and $0 \leq k<d=\operatorname{dim} P_{1}+\cdots+P_{m}$

$$
f_{k}\left(P_{1}+\cdots+P_{m}\right) \leq f_{k}\left(N_{1}+\cdots+N_{m}\right)
$$

where the family $\left(N_{1}, \ldots, N_{m}\right)$ is Minkowski neighborly with $f_{0}\left(N_{i}\right)=n_{i}$ for all $i=1, \ldots, m$. Equality holds for all $k$ if it holds for some $k_{0}, k_{0}+1 \geq \frac{d+2 m-2}{2}$.

A face of a Minkowski sum is mixed if it is the sum of positive-dimensional faces of the summands. Mixed faces play an important role in mixed volume computations and they prominently appear in toric/tropical intersection theory [FS97, Kat12, ST10], sparse resultants [PS93, EC95] as well as colorful geometric combinatorics [ABPS15] and game theory. Our methods also apply to the study of mixed faces and we establish strong upper bounds and in particular characterize the case of equality in the most important case.

Upper Bound Theorem for mixed facets. The number of mixed facets of a Minkowski sum is maximized by Minkowski neighborly families.

From discrete geometry to combinatorial topology to commutative algebra. An intriguing feature of the UBT is that its validity extends beyond the realm of convex polytopes and into combinatorial topology. Let $\Delta$ be a triangulation of the $(d-1)$-sphere and, as before, let us write $f_{k}(\Delta)$ for the number of $k$-dimensional faces. For example, boundaries of simplicial $d$-dimensional polytopes yield simplicial spheres, but these are by far not all.

Upper Bound Theorem for spheres. For a simplicial $(d-1)$-dimensional sphere $\Delta$ on $n$ vertices

$$
f_{k}(\Delta) \leq f_{k}\left(\mathrm{Cyc}_{d}(n)\right)
$$

for all $k=0,1, \ldots, d-1$. Equality holds for some $k \geq\left\lfloor\frac{d}{2}\right\rfloor$ if and only if $\Delta$ is neighborly.

The UBT for spheres was proved by Stanley [Sta75] in answer to a conjecture of Klee [Kle64] and relied on a ground-breaking connection between combinatorial topology and commutative algebra that was first described by Hochster and Reisner [Hoc77, Rei76]. To a simplicial complex $\Delta$ one associates a finitely generated graded $\mathbb{k}$-algebra $\mathbb{k}[\Delta]$ — the Stanley-Reisner ring of $\Delta$ - that algebraically encodes the simplicial complex. Hochster and Reisner showed that, in turn, algebraic properties such as Cohen-Macaulayness of $\mathbb{k}[\Delta]$ are determined by topological properties of $\Delta$. The key observation of Stanley was that enumerative properties and especially upper bounds on face numbers can be extracted from $\mathbb{k}[\Delta]$ using algebraic implications of Cohen-Macaulayness. This was the starting point of Stanley-Reisner theory. Stanley's work spawned extensions of the UBT to (pseudo-)manifolds with (mild) singularities; see for example [Nov03, Nov05, MNS11, NS12]. A pivotal result was a formula of Schenzel [Sch81] that relates algebraic properties of $\mathbb{k}[\Delta]$ to the face numbers as well as topological properties of $\Delta$, provided $\mathbb{k}[\Delta]$ is a Buchsbaum ring (which is in particular true for all manifolds).

The UBTM too will be the consequence of a statement in the topological domain that we derive using algebra, though we will also briefly comment on a geometric approach to the problem. The appropriate combinatorial/topological setup for the UBPM is that of relative simplicial complexes: A relative simplicial complex is a pair of simplicial complexes $\Psi=(\Delta, \Gamma)$ where $\Gamma \subseteq \Delta$ is a subcomplex. The faces of $\Psi$ are precisely the faces of $\Delta$ not contained in $\Gamma$. The number of $k$-dimensional faces of $\Psi$ is therefore 
$f_{k}(\Psi)=f_{k}(\Delta, \Gamma):=f_{k}(\Delta)-f_{k}(\Gamma)$. The algebraic object naturally associated to a relative complex $\Psi=(\Delta, \Gamma)$ is the Stanley-Reisner module or face module M $[\Psi]$. Upper Bounds Problems for relative complexes have been considered in different guises for instance in the study of comparison theorems for $f$-vectors [Bjö07], Upper Bound Theorems of manifolds [NS09] and polyhedra [BL81, BKL86], triangulations of polytopes [McM04], and the study of sequentially Cohen-Macaulay complexes and rings [Duv96, ABG83]. For the type of relative upper bound problems we will consider, however, it is crucial to study complexes not only under topological restrictions (such as the Buchsbaum or Cohen-Macaulay property) but to also take the combinatorics and geometry of $\Gamma$ in $\Delta$ into account. We show that relative Stanley-Reisner theory has the capacity to encode such restrictions, and exploit this fact heavily in the present paper.

Outline of the paper. We provide a gentle introduction to (relative) Stanley-Reisner theory that starts (in Section 1) with a review of the classical setup, collecting also results pertaining to relative simplicial complexes that are implicit in works of Stanley, Schenzel and others. The same applies to Section 2, where we extend the Schenzel formula to the relative setting. In Section 3 we recall Stanley's proof of the UBT for spheres which sets the stage for general relative upper bound theorems. In particular, we discuss combinatorial isoperimetric problems and the combinatorial restrictions we can impose on relative complexes.

We illustrate our methods on a variety of simplicial isoperimetric and reverse isoperimetric inequalities in parallel to the developments of the main methods. A combinatorial isoperimetric inequality bounds (from above) the size of the interior of a combinatorial object in terms of its boundary; a reverse isoperimetric problem bounds the boundary in terms of its interior.

The Schenzel formula states that the entries of the $h$-vector of $\Psi$ are given by an algebraic component $h^{\text {alg }}(\Psi)$ and a topological component $h^{\text {top }}(\Psi)$ that we study individually. The latter is typically an invariant of the problem we wish to consider, and hence the former will be of main interest to us. In Section 4 we develop several powerful tools for studying the algebraic component.

(1) Section 4.1 provides bounds by comparing a given relative complex to a simpler one. This technique recovers Stanley's approach to the UBT as a special case. The most challenging part is to characterize the case of equality which has an interesting connection to the Nerve Lemma. This approach is demonstrated in Section 4.2 for arrangements of Cohen-Macaulay subcomplexes.

(2) In Section 4.3, we integrate local information on the $h$-vector to obtain global bounds. Combined with the fact that the algebraic component $h^{\mathrm{alg}}(\Psi)$ is monotone under passing to subcomplexes, this can be used to derive effective upper bounds in many settings. This is an algebraic generalization of a geometric idea due to McMullen.

(3) The latter technique is refined in Section 4.4, to give even stronger upper bounds on the algebraic $h$-numbers; in particular, we obtain a reverse isoperimetric inequality of a kind that seems new to the subject.

(4) We close in Section 4.5 with a brief discussion of relative shellability. This technique can be used to give a combinatorial-geometric proof of the UBTM, although a proper proof is more intricate.

In Section 5 we cast the Upper Bound Problem for Minkowski sums into a relative upper bound problem. The connection to relative complexes is via Cayley polytopes. Section 5.2 illustrates the general approach for two summands and gives a simple proof for the results of Karavelas-Tzanaki [KT11]. The remainder of the section gives a complete proof of the UBTM for pure collections, that is, polytopes $P_{1}, \ldots, P_{m} \subseteq \mathbb{R}^{d}$ with $f_{0}\left(P_{i}\right) \geq d+1$ for all $i$. Section 6 treats the general case without restrictions on the number of vertices. In Section 7 we combine our results with the combinatorics of Cayley polytopes to give an upper bound on the number of mixed faces of a general sum $P_{1}+P_{2}+\cdots+P_{m}$. For mixed facets, this bound is tight, and maximized by Minkowski neighborly families.

Acknowledgements. We wish to thank the anonymous referee for a very thorough report. The first author also wants to express his gratitude to Eran Nevo for inspiring conversations and helpful comments. We are also grateful for the hospitality of the IHÉS in Bures-sur-Yvette, where most of the research leading to this paper was conducted. 


\section{Relative Stanley-Reisner theOry}

In this section we lay out the foundations for relative Stanley-Reisner theory, an algebraic-combinatorial theory for relative simplicial complexes. For further background on Stanley-Reisner rings and combinatorial commutative algebra, we refer to [Sta96] and [MS05].

A simplicial complex $\Delta$ is a collection of subsets $\Delta \subseteq 2^{[n]}$ for some $[n]:=\{1,2, \ldots, n\}$ that is closed under taking subsets. We explicitly allow $\Delta$ to be empty and we call $\varnothing$ the void complex. Thus any simplicial complex $\Delta \neq \varnothing$ contains the empty face $\varnothing$. For $S \subseteq[n]$ the simplex with vertex set $S$ is denoted by $\Delta_{S}:=2^{S}$. We also write $\Delta_{n}=\Delta_{[n]}$, and set $\Delta_{0}:=\{\varnothing\} \neq \varnothing$. A relative simplicial complex is a pair $\Psi=(\Delta, \Gamma)$ of simplicial complexes for which $\Gamma \subseteq \Delta$ is a proper subcomplex. The faces of $\Psi=(\Delta, \Gamma)$ are the elements

$$
\Delta \backslash \Gamma=\{\sigma \in \Delta: \sigma \notin \Gamma\} .
$$

An ordinary simplicial complex is thus a relative simplicial complex with $\Gamma=\varnothing$. The dimension of a relative simplicial complex is

$$
\operatorname{dim} \Psi:=\max \{\operatorname{dim} \sigma: \sigma \in(\Delta, \Gamma)\}
$$

where $\operatorname{dim} \sigma=|\sigma|-1$. We say $\Psi$ is pure if all inclusion maximal faces in $\Delta \backslash \Gamma$ are of the same dimension. The vertices of a relative complex are denoted by $\mathrm{V}(\Psi):=\{i \in[n]:\{i\} \in \Psi\}$. We write $\Psi^{(i)}$ to denote the $(i-1)$-skeleton of $\Psi$, i.e. the subcomplex of all faces of $\Psi$ of dimension $<i$.

We denote by $\widetilde{H}_{\bullet}(\Delta, \mathbb{k})$ reduced homology with coefficients in $\mathbb{k}$; unless reference to the coefficient field is necessary, we omit it. If $\Psi=(\Delta, \Gamma)$ is a relative simplicial complex, then $\widetilde{H}_{\bullet}(\Psi)=\widetilde{H}_{\bullet}(\Delta, \Gamma)$ is the usual relative homology. Observe that $\widetilde{H}_{\bullet}\left(\Delta, \Delta_{0}\right)$ is the unreduced homology of $\Delta$. The reduced Betti numbers are denoted by $\widetilde{\beta}_{i}(\Psi ; \mathbb{k})=\operatorname{dim}_{\mathbb{k}} \widetilde{H}_{i}(\Psi ; \mathbb{k})$.

Let $\mathbb{k}[\mathbf{x}]=\mathbb{k}\left[x_{1}, \ldots, x_{n}\right]$ be the polynomial ring over $\mathbb{k}$ in $n$ variables. For a monomial $\mathbf{x}^{\alpha}$ the support is defined as $\operatorname{supp}\left(\mathbf{x}^{\alpha}\right)=\operatorname{supp}(\alpha)=\left\{i \in[n]: \alpha_{i}>0\right\}$. For a simplicial complex $\Delta$ on $[n]$, the Stanley-Reisner ideal, or face ideal, is the ideal

$$
\mathrm{I}_{\Delta}:=\left\langle\mathbf{x}^{\tau}: \tau \subseteq[n], \tau \notin \Delta\right\rangle=\mathbb{k}-\operatorname{span}\left\{\mathbf{x}^{\alpha}: \operatorname{supp}\left(\mathbf{x}^{\alpha}\right) \notin \Delta\right\} \subseteq \mathbb{k}[\mathbf{x}] .
$$

The Stanley-Reisner ring or face ring is $\mathbb{k}[\Delta]:=\mathbb{k}[\mathbf{x}] / \mathrm{I}_{\Delta}$. The appropriate algebraic object associated to a relative complex $\Psi=(\Delta, \Gamma)$ is the Stanley-Reisner module or face module

$$
\mathrm{M}[\Psi]=\mathrm{M}[\Delta, \Gamma]:=\operatorname{ker}(\mathbb{k}[\Delta] \longrightarrow \mathbb{k}[\Gamma]) \cong \mathrm{I}_{\Gamma} / \mathrm{I}_{\Delta} \subseteq \mathbb{k}[\Delta]
$$

We regard $\mathrm{M}[\Psi]$ as a module over $\mathbb{k}[\mathbf{x}]$. If $\Gamma=\Delta_{0}$ is the empty complex, then $\mathrm{M}[\Psi] \cong \mathbb{k}[\Delta]$.

1.1. Face numbers and Hilbert functions. For a (relative) simplicial complex $\Psi$ of $\operatorname{dimension} \operatorname{dim} \Psi=d-1$, the $f$-vector of $\Psi$ is defined as $f(\Psi):=\left(f_{-1}, f_{0}, \ldots, f_{d-1}\right)$ where $f_{i}=f_{i}(\Psi)$ is the number of $i$-dimensional faces of $\Psi$. If $\Psi=(\Delta, \Gamma)$, then $f(\Psi)=f(\Delta)-f(\Gamma)$.

A $\mathbb{k}[\mathbf{x}]$-module $M$ is $\mathbb{Z}^{n}$-graded or finely graded if $M=\bigoplus_{\alpha \in \mathbb{Z}^{n}} M_{\alpha}$ and $\mathbf{x}^{\beta} M_{\alpha} \subseteq M_{\alpha+\beta}$ for all $\beta \in \mathbb{Z}_{\geq 0}^{n}$. For $\Psi=(\Delta, \Gamma)$, the fine Hilbert series is given by

$$
\mathrm{F}(\mathrm{M}[\Psi], \mathbf{t})=\mathrm{F}\left(\mathrm{M}[\Psi], t_{1}, \ldots, t_{n}\right):=\sum_{\alpha \in \mathbb{Z}^{n}} \operatorname{dim}_{\mathbb{k}} \mathrm{M}[\Psi]_{\alpha} \mathbf{t}^{\alpha}=\sum_{\sigma \in \Delta \backslash \Gamma} \prod_{i \in \sigma} \frac{t_{i}}{1-t_{i}} .
$$

The fine grading specializes to a $\mathbb{Z}$-grading or coarse grading and we obtain

$$
\mathrm{F}(\mathrm{M}[\Psi], t)=\sum_{\sigma \in \Delta \backslash \Gamma} \frac{t^{|\sigma|}}{(1-t)^{|\sigma|}}=\frac{\sum_{k=0}^{d} f_{k-1} t^{k}(1-t)^{d-k}}{(1-t)^{d}}=\frac{h_{0}+h_{1} t+\cdots+h_{d} t^{d}}{(1-t)^{d}} .
$$

We use the last equality as the definition of the $h$-vector $h(\Psi)=\left(h_{0}, \ldots, h_{d}\right)$ of the (relative) simplicial complex $\Psi$. If $\Psi$ is a simplicial complex, that is, if $\Gamma=\varnothing$, then $h_{0}=1$ and $h_{1}=f_{0}(\Delta)-d$. If $\operatorname{dim} \Gamma=\operatorname{dim} \Delta$, then $h(\Psi)=h(\Delta)-h(\Gamma)$ and hence $h_{0}(\Psi)=0$ and $h_{1}(\Psi)=f_{0}(\Delta)-f_{0}(\Gamma)$. The conversion between $f$-vector and $h$-vector can be made explicit as

$$
\sum_{i=0}^{d} f_{i-1}(\Psi) t^{d-i}=\sum_{k=0}^{d} h_{k}(\Psi)(t+1)^{d-k}
$$


Individual entries are thus given by

$$
h_{k}(\Psi)=\sum_{i=0}^{k}(-1)^{k-i}\left(\begin{array}{l}
d-i \\
k-i
\end{array}\right) f_{i-1}(\Psi) \text { and } f_{i-1}(\Psi)=\sum_{k=0}^{i}\left(\begin{array}{l}
d-k \\
i-k
\end{array}\right) h_{k}(\Psi) .
$$

The second formula is crucial for upper bounds on face numbers:

Observation 1.1. The number of $(i-1)$-faces $f_{i-1}(\Psi)$ is a positive linear combination of the $h$-numbers $h_{0}(\Psi), \ldots, h_{i}(\Psi)$. In particular, upper bounds on entries of the $h$-vector imply upper bounds on the $f$-vector.

Finally, the $g$-vector of the (relative) simplicial complex $\Psi$ is

$$
g(\Psi)=\left(g_{1}, \ldots, g_{d}\right):=\left(h_{1}-h_{0}, h_{2}-h_{1}, \ldots, h_{d}-h_{d-1}\right) .
$$

The link of $\sigma \subseteq[n]$ in a simplicial complex $\Delta$ is $\operatorname{lk}(\sigma, \Delta):=\{\tau \in \Delta: \sigma \cap \tau=\varnothing, \sigma \cup \tau \in \Delta\}$. In particular, $\operatorname{lk}(\varnothing, \Delta)=\Delta$ and $\operatorname{lk}(\sigma, \Delta)=\varnothing$ is the void complex whenever $\sigma \notin \Delta$. The (closed) star of a face $\sigma$ in a simplicial complex $\Delta$ is defined as $\operatorname{st}(\sigma, \Delta):=\{\tau \in \Delta: \sigma \cup \tau \in \Delta\}$ and we define the deletion of a face $\sigma$ of $\Delta$ as $\Delta-\sigma:=\{\tau \in \Delta: \sigma \nsubseteq \tau\}$. In particular, link and star of a face $\sigma$ are related by $\operatorname{lk}(\sigma, \Delta)=\operatorname{st}(\sigma, \Delta)-\sigma$. For a relative simplicial complex $\Psi=(\Delta, \Gamma)$, the notions of link and star are defined to respect the relative structure: For $\sigma \subseteq[n]$, we set $\operatorname{lk}(\sigma, \Psi)=(\operatorname{lk}(\sigma, \Delta), \operatorname{lk}(\sigma, \Gamma))$ and $\operatorname{st}(\sigma, \Psi)=(\operatorname{st}(\sigma, \Delta), \operatorname{st}(\sigma, \Gamma))$.

Lemma 1.2. Let $\Psi=(\Delta, \Gamma)$ be a relative simplicial complex. Let $v \in \mathrm{V}(\Delta)$ be any vertex and let $e-1=$ $\operatorname{dim} \operatorname{st}(v, \Psi)=\operatorname{dim} \operatorname{lk}(v, \Psi)+1$. Then

$$
h_{k}(\operatorname{lk}(v, \Psi))=h_{k}(\operatorname{st}(v, \Psi))
$$

for all $0 \leq k<e$ and $h_{e}(\operatorname{st}(v, \Psi))=0$.

Proof. Observe that $\mathrm{M}[\operatorname{st}(v, \Psi)] / x_{v} \mathrm{M}[\operatorname{st}(v, \Psi)] \cong \mathrm{M}[\mathrm{lk}(v, \Psi)]$ and that $\mathrm{M}[\operatorname{st}(v, \Psi)] \stackrel{\times x_{v}}{\longrightarrow} \mathrm{M}[\operatorname{st}(v, \Psi)]$ is injective. Passing to the coarse Hilbert series proves the claim.

The Euler characteristic of a (relative) simplicial $(d-1)$-complex $\Psi$ is

$$
\chi(\Psi):=\sum_{i=0}^{d-1}(-1)^{i} f_{i}(\Psi)=(-1)^{d-1} h_{d}(\Psi) .
$$

Notice that $\chi(\Delta)=\chi(\Delta, \varnothing)$ is the reduced Euler characteristic and $\chi(\Psi)=\chi(\Delta)-\chi(\Gamma)$.

It turns out that for various classes of simplicial complexes, the entries of the $h$-vector are not independent from each other. If $\Delta$ is a simplicial sphere, the classical Dehn-Sommerville equations state $h_{k}=h_{d-k}$ for all $k=0, \ldots, d$, a relation closely related to Poincaré duality.

The following two results are generalizations of the classical Dehn-Sommerville relations to the relative setting, to manifolds and to balls. Recall that a (relative) simplicial complex $\Psi=(\Delta, \Gamma)$ is Eulerian if $\Psi$ is pure and $\chi(\operatorname{lk}(\sigma, \Psi))=(-1)^{\operatorname{dim} \operatorname{lk}(\sigma, \Psi)}$ for all $\sigma \in \Psi$. For example, all (homology) spheres are Eulerian. For general (homology) manifolds, a weaker notion is in order. The relative complex $\Psi$ is weakly Eulerian if $\chi(\operatorname{lk}(\sigma, \Psi))=(-1)^{\operatorname{dim} \operatorname{lk}(\sigma, \Psi)}$ for all nonempty faces $\sigma$. The following lemma is a version of the Dehn-Sommerville relations (cf. [Kle64, Grä87, NS09]) for relative complexes.

Lemma 1.3 (Dehn-Sommerville relations). Let $\Psi=(\Delta, \Gamma)$ be a relative simplicial complex of dimension $d-1$. If $\Psi$ is weakly Eulerian, then

$$
h_{d-i}(\Psi)=h_{i}(\Delta)+(-1)^{i}\left(\begin{array}{l}
d \\
i
\end{array}\right)\left((-1)^{d-1} \chi(\Delta, \Gamma)-1\right) .
$$

Lemma 1.3 is a direct consequence of the following reciprocity law of $\mathrm{M}[\Psi]$.

Proposition 1.4. Let $\Psi=(\Delta, \Gamma)$ be a relative complex of dimension $d-1$. Then the fine Hilbert series of $\mathrm{M}[\Psi]$ satisfies

$$
(-1)^{d} \mathrm{~F}\left(\mathrm{M}[\Psi], \frac{1}{t_{1}}, \ldots, \frac{1}{t_{n}}\right)=\sum_{a \in \mathbb{Z}_{\geq 0}^{n}}(-1)^{\operatorname{codim} \sigma_{a}} \chi\left(\operatorname{lk}\left(\sigma_{a}, \Psi\right)\right) \mathbf{t}^{a}
$$

where $\sigma_{a}=\operatorname{supp}(a)$ and $\operatorname{codim} \sigma_{a}:=\operatorname{dim} \Psi-\operatorname{dim} \sigma_{a}$. 
This is a statement analogous to Lemma 5.4.3 in [BH93]. If $\Psi=(\Delta, \Gamma)$ is weakly Eulerian, then Proposition 1.4 yields

$$
(-1)^{d} \mathrm{~F}\left(\mathrm{M}[\Psi], \frac{1}{\mathbf{t}}\right)=(-1)^{d-1} \chi(\Delta, \Gamma)-1+\mathrm{F}(\mathrm{M}[\Delta], \mathbf{t}) .
$$

Passing to the coarse Hilbert series proves Lemma 1.3.

1.2. Cohen-Macaulay and Buchsbaum modules and complexes. Let $M$ be a finitely generated graded module over $\mathbb{k}[\mathbf{x}]$. For a sequence $\Theta=\left(\theta_{1}, \ldots, \theta_{\ell}\right)$ of elements of $\mathbb{k}[\mathbf{x}]$, let us write $\Theta_{s}=\left(\theta_{1}, \ldots, \theta_{s}\right)$ for the subsequence of the first $s$ elements and $\Theta_{s} M=\sum_{i=1}^{s} \theta_{i} M$. A partial homogeneous system of parameters (partial h.s.o.p.) is a sequence $\Theta=\left(\theta_{1}, \ldots, \theta_{\ell}\right)$ of homogeneous elements of $\mathbb{k}[\mathbf{x}]$ such that

$$
\operatorname{dim} M / \Theta M=\operatorname{dim} M-\ell .
$$

If $\ell=\operatorname{dim} M$, then $\Theta$ is a homogeneous system of parameters. If all $\theta_{i}$ are of degree one, then $\Theta$ is a (partial) linear system of parameters (l.s.o.p.). Throughout the paper we assume that the field $\mathbb{k}$ is infinite, which guarantees the existence of a linear system of parameters (cf. [Eis95, Theorem. 13.3]).

A sequence $\Theta=\left(\theta_{1}, \ldots, \theta_{\ell}\right)$ of homogeneous elements is called $M$-regular, if $\Theta M \neq M$ and

$$
\Theta_{i-1} M: \theta_{i}:=\left\{m \in M: m \theta_{i} \in \Theta_{i-1} M\right\}=\Theta_{i-1} M
$$

for all $i=1, \ldots, \ell$. Every regular sequence is a partial h.s.o.p. but the converse is false. An immediate consequence of the definition is the following.

Proposition 1.5. Let $M$ be a finitely generated graded module over $\mathbb{k}[\mathbf{x}]$ and $\Theta=\left(\theta_{1}, \ldots, \theta_{r}\right)$ be an $M$-regular sequence of linear forms. Then $(1-t)^{r} \mathrm{~F}(M, t)=\mathrm{F}(M / \Theta M, t)$.

The length of the longest regular sequence of a module $M$ is called the $\operatorname{depth} \operatorname{depth}(M)$ of $M$. Clearly, $\operatorname{depth}(M) \leq \operatorname{dim}(M)$, and Cohen-Macaulayness characterizes the case of equality.

Definition 1.6. A $\mathbb{k}[\mathbf{x}]$-module $M$ is called Cohen-Macaulay if $\operatorname{depth}(M)=\operatorname{dim}(M)$. A relative simplicial complex $\Psi=(\Delta, \Gamma)$ is Cohen-Macaulay (CM, for short) if $\mathrm{M}[\Psi]$ is a Cohen-Macaulay module.

To treat Upper Bound Problems on manifolds, we will need to consider a more general class of complexes. Let us denote by $\mathfrak{m}:=\left\langle x_{1}, \ldots, x_{n}\right\rangle \subseteq \mathbb{k}[\mathbf{x}]$ the irrelevant ideal. An h.s.o.p. $\Theta=\left(\theta_{1}, \ldots, \theta_{\ell}\right)$ is a weak $M$-sequence if $\Theta M \neq M$ and

$$
\Theta_{i-1} M: \theta_{i}=\Theta_{i-1} M: \mathfrak{m}
$$

for all $i=1, \ldots, \ell$.

Definition 1.7. A finitely generated graded module $M$ over $\mathbb{k}[\mathbf{x}]$ is Buchsbaum if every h.s.o.p. is a weak $M$-sequence. A relative simplicial complex $\Psi$ is Buchsbaum if the face module $\mathrm{M}[\Psi]$ is Buchsbaum.

Every Cohen-Macaulay module is also a Buchsbaum module. The converse is clearly false, as will become clear once we provide the topological criterion for Cohen-Macaulay and Buchsbaum complexes.

The depth of a module is detected by local cohomology. We denote by $H_{\mathfrak{m}}^{i}(M)$ the $i$-th local cohomology of $M$ with support in $\mathfrak{m}$, cf. [ILL $\left.{ }^{+} 07\right]$. In the case of a face module, the $\mathbb{Z}^{n}$-graded Hilbert-Poincaré series can be computed in terms of local topological information of the relative simplicial complex. The following is a relative version of a formula due to Hochster; see [Sta96, Theorem II.4.1] or [MS05, Theorem 13.13].

Theorem 1.8 (Hochster's formula for relative complexes). Let $\Psi=(\Delta, \Gamma)$ be a relative simplicial complex and let $\mathrm{M}=\mathrm{M}[\Psi]$ denote its face module. The Hilbert series of the local cohomology modules in the fine grading is

$$
\mathrm{F}\left(H_{\mathfrak{m}}^{i}(\mathrm{M}), \mathbf{t}\right)=\sum_{\sigma \in \Delta} \operatorname{dim}_{\mathbb{k}} \widetilde{H}_{i-\operatorname{dim} \sigma-2}(\operatorname{lk}(\sigma, \Psi)) \prod_{i \in \sigma} \frac{t_{i}^{-1}}{1-t_{i}^{-1}} .
$$

In other words, for $\alpha \in \mathbb{Z}^{n}$ and $\sigma_{\alpha}:=\operatorname{supp}(\alpha)$, we have

$$
H_{\mathfrak{m}}^{i}(\mathrm{M})_{\alpha} \cong \begin{cases}\widetilde{H}_{i-\operatorname{dim} \sigma-2}\left(\operatorname{lk}\left(\sigma_{\alpha}, \Psi\right)\right) & \text { if } \alpha \leq 0 \text { and } \sigma_{\alpha} \in \Delta, \text { and } \\ 0 & \text { otherwise. }\end{cases}
$$


Proof. Following the proof of [MS05, Theorem 13.13], let $\check{C}=\bigoplus_{\sigma \subseteq[n]} \mathbb{k}[\mathbf{x}]_{\mathbf{x}^{\sigma}}$ be the $\mathbb{Z}^{n}$-graded Čech complex with respect to $x_{1}, \ldots, x_{n}$ where $\mathbb{k}[\mathbf{x}]_{\mathbf{x}^{\sigma}}$ is the localization at $\mathbf{x}^{\sigma}$. By definition of $\mathrm{M}=\mathrm{M}[\Psi]$, we have the short exact sequence of $\mathbb{Z}^{n}$-graded modules

$$
0 \longrightarrow \mathrm{M} \longrightarrow \mathbb{k}[\Delta] \longrightarrow \mathbb{k}[\Gamma] \longrightarrow 0
$$

Let $\alpha \in \mathbb{Z}^{n}$ be arbitrary but fixed and let $\alpha^{+}, \alpha^{-} \in \mathbb{Z}_{\geq 0}^{n}$ such that $\alpha=\alpha^{+}-\alpha^{-}$and $\operatorname{supp}\left(\alpha^{+}\right) \cap \operatorname{supp}\left(\alpha^{-}\right)=\varnothing$. Moreover let $\sigma=\operatorname{supp}(\alpha)$. From the proof of Theorem 13.13 in [MS05], we know that the complex of $\mathbb{k}_{\text {-vector }}$ spaces $(\mathbb{k}[\Delta] \otimes \check{C})_{\alpha}$ is isomorphic to the chain complex of $\operatorname{lk}(\sigma, \Delta)$ shifted by $\left|\operatorname{supp}\left(\alpha^{-}\right)\right|+1$ if $\alpha^{+}=0$ and acyclic otherwise. The argument applies to $(\mathbb{k}[\Gamma] \otimes \check{C})_{\alpha}$ as well and thus $(\mathrm{M} \otimes \check{C})_{\alpha}$ is isomorphic to the chain complex of the pair $\operatorname{lk}(\sigma, \Psi)=\left(\operatorname{lk}(\sigma, \Delta, \operatorname{lk}(\sigma, \Gamma))\right.$, again shifted by $\left|\operatorname{supp}\left(\alpha^{-}\right)\right|+1$ if $\alpha^{+}=0$. It follows that as $\mathbb{k}$-vector spaces $H_{\mathfrak{m}}^{i}(\mathrm{M})_{\alpha} \cong \widetilde{H}_{i-\operatorname{dim} \sigma-2}\left(\operatorname{lk}\left(\sigma_{\alpha}, \Psi\right)\right)$ whenever $\alpha \leq 0$ and identically zero otherwise.

From the relative Hochster formula and the fact that $H_{\mathfrak{m}}^{i}(M)=0$ for $i<e=\operatorname{depth}(M)$ and $H_{\mathfrak{m}}^{e}(M) \neq 0$ we deduce a relative version of a criterion of Reisner [Rei76] for simplicial complexes to be Cohen-Macaulay.

Theorem 1.9 ([Sta96, Theorem III.7.2]). A relative simplicial complex $\Psi=(\Delta, \Gamma)$ is Cohen-Macaulay if and only if $\widetilde{H}_{i}(\operatorname{lk}(\sigma, \Psi))=0$ for all faces $\sigma \in \Delta$ and all $i<\operatorname{dim} \operatorname{lk}(\sigma, \Psi)$.

Corollary 1.10 ([Sta96, Theorem III.7.3]). Let $\Delta$ be a simplicial complex and $\Gamma \subseteq \Delta$ a subcomplex. If $\Delta$ and $\Gamma$ are Cohen-Macaulay and $\operatorname{dim} \Delta-\operatorname{dim} \Gamma \leq 1$, then $(\Delta, \Gamma)$ is Cohen-Macaulay. Conversely, if $\Delta$ and $(\Delta, \Gamma)$ are Cohen-Macaulay then $\operatorname{dim} \Delta-\operatorname{dim} \Gamma \leq 1$ and $\widetilde{H}_{i}(\operatorname{lk}(\sigma, \Gamma))=0$ for all $\sigma \in \Gamma$ and $i<\operatorname{dim} \Delta-\operatorname{dim} \sigma-2$.

Proof. Let us write $\operatorname{lk}(\sigma, \Psi)=\left(\Delta_{\sigma}, \Gamma_{\sigma}\right)$ for $\sigma \in \Delta$. For the pair $\left(\Delta_{\sigma}, \Gamma_{\sigma}\right)$ consider the long exact sequence

$$
\cdots \longrightarrow \widetilde{H}_{i}\left(\Delta_{\sigma}\right) \longrightarrow \widetilde{H}_{i}\left(\Delta_{\sigma}, \Gamma_{\sigma}\right) \longrightarrow \widetilde{H}_{i-1}\left(\Gamma_{\sigma}\right) \longrightarrow \widetilde{H}_{i-1}\left(\Delta_{\sigma}\right) \longrightarrow \widetilde{H}_{i-1}\left(\Delta_{\sigma}, \Gamma_{\sigma}\right) \longrightarrow \cdots
$$

The vanishing of homologies splits the sequence and the first claim follows from

$$
0=\widetilde{H}_{i}\left(\Delta_{\sigma}\right) \rightarrow \widetilde{H}_{i}\left(\Delta_{\sigma}, \Gamma_{\sigma}\right) \rightarrow \widetilde{H}_{i-1}\left(\Gamma_{\sigma}\right)=0 \text { for } i<\operatorname{dim}\left(\Delta_{\sigma}, \Gamma_{\sigma}\right) \leq \operatorname{dim} \Delta_{\sigma} .
$$

The second claim follows analogously.

A similar criterion can be derived to characterize (relative) Buchsbaum complexes; cf. [Miy89, Sch81].

Theorem 1.11. For a pure relative simplicial complex $\Psi=(\Delta, \Gamma)$ of dimension $d-1$ the following are equivalent:

(i) $\Psi$ is a Buchsbaum complex.

(ii) $\mathrm{M}[\Psi]$ is a Buchsbaum module.

(iii) The link of every vertex is Cohen-Macaulay. ( $\Psi$ is locally Cohen-Macaulay.)

(iv) For every nonempty face $\sigma$ of $\Delta$ and all $i<d-\operatorname{dim} \sigma-1$, we have $\widetilde{H}_{i}(\operatorname{lk}(\sigma, \Psi))=0$.

Proof. (i) $\Leftrightarrow$ (ii) is true by definition. The equivalence (iii) $\Leftrightarrow$ (iv) is Theorem 1.9. Assuming (ii), we obtain from equation (2) that every localization $\mathrm{M}[\Psi]_{\mathfrak{p}}$ at primes $\mathfrak{p} \neq \mathfrak{m}$ yields a Cohen-Macaulay module. The implication (ii) $\Rightarrow$ (iii) now follows from the same argument as in [Rei76, Lemma 5] applied to face modules. Finally, assuming (iv), it follows from Theorem 1.8 that $H_{\mathfrak{m}}^{i}(\mathrm{M}[\Psi])_{j}=0$ whenever $j \neq 0$ and $0 \leq i \leq \operatorname{dim} \Psi$ and [Sch82, Satz 4.3.1] assures us that $\mathrm{M}[\Psi]$ is Buchsbaum.

An immediate corollary from the topological characterizations is that the Cohen-Macaulay and Buchsbaum properties are inherited to skeleta.

Corollary 1.12. The k-skeleton of a relative Cohen-Macaulay or Buchsbaum complex is Cohen-Macaulay or Buchsbaum, respectively. 


\section{Local COHOmology of Buchsbaum face modules}

A key observation in Stanley's proof of the UBT for spheres $\Delta$ is that

$$
\mathrm{F}(\mathbb{k}[\Delta] / \Theta \mathbb{k}[\Delta], t)=h_{0}(\Delta)+h_{1}(\Delta) t+\cdots+h_{d}(\Delta) t^{d}
$$

if $\Theta$ is a l.s.o.p. of length $d=\operatorname{dim} \Delta+1$. However, this line of reasoning fails for general manifolds or Buchsbaum complexes. For these cases, an important tool was developed by Schenzel [Sch81] that takes into account the topological/homological properties of $\Delta$. In this section, we prove a generalization of Schenzel's formula to relative complexes. Our treatment is tailor-made for Stanley-Reisner modules and slightly simpler than Schenzel's original approach.

A criterion of Schenzel [Sch82, Satz 4.3.1] states that a graded $\mathbb{k}[\mathbf{x}]$-module $M$ is Buchsbaum whenever the $\mathbb{Z}$-graded local cohomology $H_{\mathfrak{m}}^{i}(M)$ is concentrated in a fixed degree for all $0 \leq i<\operatorname{dim} M$. Schenzel [Sch81] showed that the converse holds for special classes of Buchsbaum modules. For this, he used the purity of the Frobenius based on earlier work of Hochster and Roberts [HR74]. For Stanley-Reisner modules, the concentration of local cohomology is a consequence of Theorem 1.8:

Corollary 2.1. Let $\Psi=(\Delta, \Gamma)$ be a pure relative simplicial complex of dimension $d-1$. Then the following are equivalent:

(i) $\widetilde{H}_{i}(\operatorname{lk}(\sigma, \Psi))=0$ for all non-empty faces $\sigma \in \Delta$ and all $i<\operatorname{dim} \operatorname{lk}(\sigma, \Delta)=d-\operatorname{dim} \sigma-1$,

(ii) The coarse-graded local cohomology of $\Psi$ is concentrated in degree zero, that is, $H_{\mathfrak{m}}^{i}(\mathrm{M}[\Psi])_{j}=0$ for all $i, j, j \neq 0$.

(iii) $\mathrm{M}[\Psi]$ is a Buchsbaum module.

For the following discussion, let us write $R=\mathbb{k}[\mathbf{x}]$ and define $R(t)$ to be the free rank-one $R$-module generated in degree $-t$. Recall that for an homogeneous element $\theta_{1} \in R_{t}$ the Koszul complex $K\left(\theta_{1}\right)$ is the complex

$$
0 \longrightarrow R \stackrel{\times \theta_{1}}{\longrightarrow} R(t) \longrightarrow 0 \text {. }
$$

For family of homogeneous elements $\Theta=\left(\theta_{1}, \theta_{2}, \ldots, \theta_{n}\right)$ the Koszul complex is defined as

$$
K(\Theta)=K\left(\theta_{1}\right) \otimes_{R} K\left(\theta_{2}\right) \otimes_{R} \cdots \otimes_{R} K\left(\theta_{n}\right) .
$$

For a graded $R$-module $M$ we denote by $H^{\bullet}(\Theta ; M):=H^{\bullet}\left(K(\Theta) \otimes_{R} M\right)$ the Koszul cohomology of $M$ with respect to $\Theta$. The Koszul complex is a basic tool in the study of local cohomology and we refer the reader to $\left[\mathrm{BH} 93, \mathrm{ILL}^{+} 07\right]$ for further information.

Lemma 2.2. Let $M$ be a Buchsbaum module of dimension $d$ and $\Theta=\left(\theta_{1}, \ldots, \theta_{d}\right)$ a h.s.o.p. for $M$. Then for all $0 \leq i<d, \mathfrak{m} H^{i}(\Theta ; M)=0$. In particular, the Koszul cohomology modules are $\mathbb{k}$-vector spaces of dimension

$$
\operatorname{dim}_{\mathbb{k}} H^{i}\left(\Theta_{s} ; M\right)=\sum_{j=0}^{i}\left(\begin{array}{c}
s \\
i-j
\end{array}\right) \operatorname{dim}_{\mathfrak{k}} H_{\mathfrak{m}}^{j}(M) .
$$

The lemma is a direct consequence of Lemma 4.2.1 of [Sch82] except that we need to verify that $H^{i}\left(\Theta_{s} ; M\right)$ is of finite length for all $i$.

Proposition 2.3. If $M$ is a Buchsbaum module and $\Theta$ is a h.s.o.p., then $H^{i}\left(\Theta_{s} ; M\right)$ is of finite length for all $0 \leq i \leq s$.

Proof. For $s=d:=\operatorname{dim} M, \Theta$ is a homogeneous system of parameters. Thus $H^{d}(\Theta ; M)=M / \Theta M$ is of dimension zero, and therefore of finite length and the result follows. For $s<d$, observe that for all $j \geq 1$, $\Theta^{\prime}=\left(\theta_{1}, \ldots, \theta_{s}, \theta_{s+1}^{j}\right)$ is a partial h.s.o.p. for M. Now, $K\left(\Theta^{\prime}\right)$ is the mapping cone of $\times \theta_{s+1}^{j}: K\left(\Theta_{s}\right) \longrightarrow K\left(\Theta_{s}\right)$ and the long exact sequence in cohomology yields

$$
0 \longrightarrow H^{i-1}\left(\Theta_{s} ; \mathrm{M}\right) / \theta_{s}^{j+1} H^{i-1}\left(\Theta_{s} ; \mathrm{M}\right) \longrightarrow H^{i}\left(\Theta^{\prime} ; \mathrm{M}\right)
$$

By induction on $s$ and Lemma $2.2, \mathfrak{m} H^{i-1}\left(\Theta_{s} ; \mathrm{M}\right)=0$ since $H^{i}\left(\Theta^{\prime} ; \mathrm{M}\right)$ is annihilated by the irrelevant ideal for all $j \geq 1$. The finite length of $H^{i-1}\left(\Theta_{s} ; \mathrm{M}\right)$ now follows from Nakayama's Lemma.

As a consequence we note the following. 
Corollary 2.4. Let $M$ be Buchsbaum R-module of dimension d and let $\Theta$ be a l.s.o.p. for $M$. Then for all $0<s \leq d$, we have

$$
\operatorname{dim}_{\mathbb{k}}\left(\Theta_{s-1} M: \theta_{s}\right) /\left(\Theta_{s-1} M\right)=\sum_{i=0}^{s-1}\left(\begin{array}{c}
s-1 \\
i
\end{array}\right) \operatorname{dim}_{\mathbb{k}} H_{\mathfrak{m}}^{i}(M) .
$$

Proof. As in [Sch82, Lemma 6.3.4], we have the short exact sequence

$$
0 \longrightarrow H^{s-2}\left(\Theta_{s-1} ; M\right) \longrightarrow H^{s-1}\left(\Theta_{s} ; M\right) \longrightarrow\left(\Theta_{s-1} M: \theta_{s}\right) /\left(\Theta_{s-1} M\right) \longrightarrow 0
$$

as $H^{s-1}\left(\Theta_{s} ; M\right)$ is annihilated by the irrelevant ideal. By Lemma 2.2 and the Buchsbaum property (2), this is a sequence of $\mathbb{k}$-vector spaces and hence splits. The result now follows from Lemma 2.2.

With this, we can reprove and generalize Schenzel's Formula to relative Buchsbaum complexes, and therefore provide a central tool for relative Stanley-Reisner Theory.

Theorem 2.5 (Relative Schenzel Formula). Let $\Psi=(\Delta, \Gamma)$ be a relative Buchsbaum complex of dimension $d-1$ and $\mathrm{M}=\mathrm{M}[\Psi]$ the associated face module. If $\Theta$ is a l.s.o.p. for $\mathrm{M}$, then

$$
(1-t)^{d} \mathrm{~F}(\mathrm{M}, t)=\mathrm{F}(\mathrm{M} / \Theta \mathrm{M}, t)+\sum_{j=1}^{d}\left(\begin{array}{l}
d \\
j
\end{array}\right)\left(\sum_{i=0}^{j-1}(-1)^{j-i} \widetilde{\beta}_{i-1}(\Delta, \Gamma)\right) t^{j} .
$$

The formula states that the $h$-vector of a relative complex $\Psi$ is the sum of an algebraic and a topological component. In this spirit, we denote the algebraic component by

$$
h_{k}^{\mathrm{alg}}(\Psi):=\operatorname{dim}_{\mathbb{k}}(\mathrm{M}[\Delta, \Gamma] / \Theta \mathrm{M}[\Delta, \Gamma])_{k},
$$

where $\Theta$ is a l.s.o.p. and the topological component by

$$
h_{k}^{\text {top }}(\Psi):=\left(\begin{array}{l}
d \\
k
\end{array}\right) \sum_{i=0}^{k-1}(-1)^{k-i} \widetilde{\beta}_{i-1}(\Delta, \Gamma) .
$$

We can rewrite the relative Schenzel formula as follows.

Theorem 2.5. Let $\Psi=(\Delta, \Gamma)$ be a relative Buchsbaum complex of dimension d-1. If $\Theta$ is a l.s.o.p. for $\mathrm{M}=\mathrm{M}[\Psi]$, then

$$
h_{k}(\Psi)=h_{k}^{\mathrm{alg}}(\Psi)+h_{k}^{\mathrm{top}}(\Psi)
$$

for any $k$.

Proof. We closely follow Schenzel's proof of the case of simplicial complexes [Sch81, Theorem 4.3]. For a linear form $\theta \in \mathbb{k}[\mathbf{x}]$ and a graded $\mathbb{k}[\mathbf{x}]$-module $N$ we have the exact sequence

$$
0 \longrightarrow\left(0_{N}: \theta\right) \longrightarrow N \stackrel{\theta}{\longrightarrow} N(1) \longrightarrow(N / \theta N)(1) \longrightarrow 0
$$

and hence

$$
(1-t) \mathrm{F}(N, t)=\mathrm{F}(N / \theta N, t)-t \mathrm{~F}\left(\left(0_{N}: \theta\right), t\right) .
$$

Iterating this argument with $N=\mathrm{M}[\Psi] / \Theta_{s} \mathrm{M}[\Psi]$ for $1 \leq s \leq d$, this yields

$$
(1-t)^{d} \mathrm{~F}(\mathrm{M}, t)=\mathrm{F}(\mathrm{M} / \Theta \mathrm{M}, t)-\sum_{s=1}^{d} t(1-t)^{s-1} \mathrm{~F}\left(\left(\Theta_{s-1} \mathrm{M}: \theta_{s}\right) / \Theta_{s-1} \mathrm{M}, t\right) .
$$

By Corollary 2.4 and Hochster's formula, we finally obtain

$$
\mathrm{F}\left(\left(\Theta_{s-1} \mathrm{M}: \theta_{s}\right) / \Theta_{s-1} \mathrm{M}, t\right)=\sum_{i=0}^{d-s}\left(\begin{array}{c}
d-s \\
i
\end{array}\right) \operatorname{dim}_{\mathrm{k}} \widetilde{\beta}_{i-1}(\Psi) t^{i} .
$$




\section{Relative Upper Bound Problems}

In this section we lay out model problems for relative upper bounds that will be addressed with relative Stanley-Reisner theory and in particular relative Buchsbaum complexes. We start by discussing the classical Upper Bound Theorem for polytopes and spheres. We address two combinatorial isoperimetric problems that allow us to introduce the notion of full subcomplexes. The proofs of the respective upper bounds are postponed to Section 4.2 where general techniques will be available.

3.1. The Upper Bound Theorem for spheres. In the proof of the UBT for polytopes, the first step is to reduce the problem of finding a $d$-polytope on $n$ vertices that maximizes the number of $k$-faces to a problem about simplicial $(d-1)$-spheres by observing that by perturbing the vertices of a polytope, the number of faces can only increase, cf. [Kle64]. In light of Observation 1.1, it is now sufficient to bound the $h$-vector of $\Delta$. The crucial lemma due to Stanley is the following.

Lemma 3.1. Let $\Delta$ be a $(d-1)$-dimensional Cohen-Macaulay complex on $n$ vertices. Then

$$
h_{k}(\Delta) \leq\left(\begin{array}{c}
n-d-1+k \\
k
\end{array}\right)
$$

for all $0 \leq k \leq d$. Equality holds for some $k_{0}$ if and only if $\Delta$ has no non-face of dimension $<k_{0}$.

Proof. For a linear system of parameters $\Theta$, Proposition 1.5 yields

$$
H(\mathbb{k}[\Delta] / \Theta \mathbb{k}[\Delta], t)=h_{0}+h_{1} t+\cdots+h_{d} t^{d}
$$

where $\left(h_{0}, \ldots, h_{d}\right)$ is the $h$-vector of $\Delta$. We have the canonical graded surjection $N:=\mathbb{k}\left[x_{1}, \ldots, x_{n}\right] \rightarrow \mathbb{k}[\Delta]$ as $\mathbb{k}[\mathbf{x}]$-modules. Now, $\Theta$ is a regular sequence for $N$ and $N / \Theta N \cong \mathbb{k}\left[y_{1}, \ldots, y_{h_{1}}\right]$ with $h_{1}=n-d$. We obtain

$$
h_{k}(\Delta)=\operatorname{dim}_{\mathbb{k}}(\mathbb{k}[\Delta] / \Theta \mathbb{k}[\Delta])_{k} \leq \operatorname{dim}_{\mathbb{k}} \mathbb{k}\left[y_{1}, \ldots, y_{h_{1}}\right]_{k}=\left(\begin{array}{c}
n-d+k-1 \\
k
\end{array}\right)
$$

for all $0 \leq k \leq d$ which completes the proof of the inequality. For the equality case, we may assume that $\Delta$ is not the $(n-1)$-simplex and thus $I_{\Delta} \neq 0$. By [BH93, Proposition 1.1.4] we have the short exact sequence

$$
0 \longrightarrow \mathrm{I}_{\Delta} / \Theta \mathrm{I}_{\Delta} \longrightarrow \mathbb{k}[\mathbf{x}] / \Theta \mathbb{k}[\mathbf{x}] \longrightarrow \mathbb{k}[\Delta] / \Theta \mathbb{k}[\Delta] \longrightarrow 0 \text {. }
$$

Equality holds for $k_{0}$ if and only if $\mathrm{I}_{\Delta} / \Theta \mathrm{I}_{\Delta}$ and hence $I_{\Delta}$ has no generators in degrees $\leq k_{0}$.

In fact stronger relations hold for the $h$-vector of a Cohen-Macaulay complex. The following type of inequalities will be the subject of Section 4.3; see Example 4.19 for the proof.

Proposition 3.2. Let $\Delta$ be a $(d-1)$-dimensional Cohen-Macaulay complex. Then

$$
k h_{k}(\Delta) \leq(n-d+k-1) h_{k-1}(\Delta)
$$

for all $k=1, \ldots, d$. In particular, $h_{k}(\Delta) \leq\left(\begin{array}{c}n-d+k-1 \\ k\end{array}\right)$ and

$$
g_{k}(\Delta)=h_{k}(\Delta)-h_{k-1}(\Delta)=h_{k-1}(\Delta)\left(\frac{h_{k}(\Delta)}{h_{k-1}(\Delta)}-1\right) \leq\left(\begin{array}{c}
n-d+k-2 \\
k
\end{array}\right) .
$$

Lemma 3.1 together with Reisner's criterion (Theorem 1.9) now implies upper bounds on the first half of the $h$-vector. The bounds are tight for boundary complexes of neighborly polytopes. The second half of $h(P)$ is taken care of by the Dehn-Sommerville equations which apply as $\partial P$ is Eulerian. This enabled Stanley [Sta75] to generalize McMullen's Upper Bound Theorem [McM70] from polytopes to simplicial spheres.

Theorem 3.3 (Upper Bound Theorem for spheres). If $\Delta$ is a simplicial $(d-1)$-sphere on $n$ vertices, then

$$
h_{k}(\Delta)=h_{d-k}(\Delta) \leq\left(\begin{array}{c}
n-d+k-1 \\
k
\end{array}\right)
$$

for all $0 \leq k \leq\left\lfloor\frac{d}{2}\right\rfloor$. Moreover, the h-vector is maximized precisely on neighborly $(d-1)$-spheres.

For more on neighborly polytopes and McMullen's geometric perspective on the upper bound theorem, we refer the reader to Section 8 of Ziegler's book [Zie95]. 
3.2. Combinatorial isoperimetric problems. The classical isoperimetric problem asks for the maximum volume of a $d$-dimensional convex body $K$ with an upper bound on the surface area. The following is a suitable discrete analog.

Question 3.4 (Combinatorial Isoperimetric Problem). Let $\Delta$ be a triangulation of a d-ball on $m+n$ vertices and $n$ vertices in the boundary. What is the maximal number of $k$-faces in the interior of $\Delta$ ?

This is a model problem for relative complexes. We seek to maximize $f_{k}(\Delta, \partial \Delta)=f_{k}(\Delta)-f_{k}(\partial \Delta)$. As it turns out a resolution to the combinatorial isoperimetric problem can be given using the "classical" tools of Section 1, provided that we make the additional assumption that the Generalized Lower Bound Conjecture of McMullen and Walkup [MW71] holds for $\Gamma=\partial \Delta$, that is, $g_{k}(\Gamma)=h_{k}(\Gamma)-h_{k-1}(\Gamma) \geq 0$ for all $k$. For a relative complex $\Psi=(\Delta, \Gamma)$ with $\Gamma \neq \varnothing$, we have $h_{0}(\Psi)=0$ and $h_{1}(\Psi)=f_{0}(\Delta)-f_{0}(\Gamma)$ and we need only to worry about $h_{k}(\Psi)$ for $k \geq 2$.

Theorem 3.5 (Combinatorial isoperimetry of balls I). Let $\Delta$ be a simplicial $(d-1)$-ball on $m+n$ vertices with $n$ vertices in the boundary and assume that the Generalized Lower Bound Conjecture holds for $\partial \Delta$. Then the following inequalities hold:

(a) For $2 \leq k \leq \frac{d}{2}$

$$
h_{k}(\Delta, \partial \Delta) \leq\left(\begin{array}{c}
m+n-d+k-1 \\
k
\end{array}\right) .
$$

Equality holds for some $k_{0} \leq \frac{d}{2}$ if and only if every non-face $\sigma$ of $\Delta$ of dimension $<k_{0}$ is supported in $\partial \Delta$. (b) For $\frac{d}{2}<k \leq d$

$$
h_{k}(\Delta, \partial \Delta) \leq\left(\begin{array}{c}
m+n-1-k \\
d-k
\end{array}\right) .
$$

Equality holds for some $k_{0}>\frac{d}{2}$ if and only if $\Delta$ has no non-face $\sigma$ of dimension $<d-k_{0}$.

Moreover, the bounds are tight: For every $n \geq d \geq 0$ and $m \geq 0$, there is a $(d-1)$-ball that attains the upper bounds for every $k$ simultaneously.

Proof. For (a), notice that

$$
h_{k}(\Delta, \partial \Delta)=h_{k}(\Delta)-g_{k}(\partial \Delta) \leq\left(\begin{array}{c}
m+n-d+k-1 \\
k
\end{array}\right)-g_{k}(\partial \Delta)
$$

by Lemma 3.1. The Generalized Lower Bound Conjecture for $\partial \Delta$ yields the claim.

For part (b), notice that by the Dehn-Sommerville relations (Lemma 1.3), we have $h_{k}(\Delta, \partial \Delta)=h_{d-k}(\Delta)$ for all $0 \leq k \leq d$ and we can again appeal to Lemma 3.1.

For tightness, let us consider any cyclic $(d-1)$-sphere $N$ on $m+n$ vertices. Now, using the method of Billera-Lee [BL80], we may find a stacked $(d-1)$-ball $B \subseteq N$ on $n$ vertices. With this, we can set $\Delta$ as the subcomplex of $N$ induced by the facets not in $B$. Then, $\Delta$ is neighborly, and $\partial \Delta$ is stacked. Therefore, all inequalities above are attained with equality.

3.3. Combinatorial isoperimetry II: Full complexes. One of the key features of relative Stanley-Reisner Theory is that we can impose restrictions on the 'position' of $\Gamma$ in $\Delta$. A profitable way to encode such a positional restriction of $\Gamma$ is the notion of full subcomplexes:

Definition 3.6. A subcomplex $\Gamma \subseteq \Delta$ is full if every face of $\Delta$ whose vertices are contained in $\Gamma$ is also a face of $\Gamma$. We call the relative complex $\Psi=(\Delta, \Gamma)$ full if $\Gamma$ is full in $\Delta$.

The notion of full subcomplex generalizes the idea of vertex-induced subgraphs. This is a very natural notion that makes prominent appearances in PL topology [Zee66, RS72], algebraic topology [Geo08, JMR83], graph theory, commutative algebra [Hoc77] and geometric group theory [CD95, Dav08].

While it may seem quite restrictive to consider only full subcomplexes, we shall later see that the notion can be refined effectively using the more flexible notion of "full arrangements", compare Section 4.1. 
Proposition 3.7. Let $\Delta$ denote any simplicial complex, and let $\Gamma$ denote any subcomplex. The following are equivalent:

(i) $\Gamma$ is full in $\Delta$;

(ii) For every face $F \in \Delta$ with $\partial F \in \Gamma$, we have $F \in \Gamma$;

(iii) $\Gamma=\Delta \cap \Delta_{\mathrm{V}(\Gamma)}$.

Notice that fullness is not a topological invariant; it is preserved under subdivisions, but not under PL homeomorphisms. The notion of fullness is, for instance, useful when identifying two simplicial complexes along a common subcomplex. Fullness then guarantees that the result is again a simplicial complex. Hence, the notion of fullness can in particular be used to bound the complexity of PL handlebodies.

Theorem 3.8 (Combinatorial isoperimetry for manifolds). Let $M$ denote a simplicial $(d-1)$-manifold on $m+n$ vertices, and let $B$ denote a $(d-2)$-dimensional submanifold on $n$ vertices of $\partial M$ such that $B$ is full in $M$. Then

$$
h_{k}(M, B) \leq\left(\begin{array}{c}
m+n-d+k-1 \\
k
\end{array}\right)-\left(\begin{array}{c}
n-d+k-1 \\
k
\end{array}\right)+\left(\begin{array}{l}
d \\
k
\end{array}\right) \sum_{i=0}^{k-1}(-1)^{k-i} \widetilde{\beta}_{i-1}(M, B)
$$

for all $0 \leq k \leq d$.

By Theorem 1.11, $\Psi=(M, B)$ is a relative Buchsbaum complex and we can use Theorem 2.5 to upper bound $h_{k}(\Psi)$. We postpone the estimation on the algebraic component to Section 4 where the necessary tools are developed. In the case when $M$ is a $d$-ball and $B$ is the bounding $(d-1)$-sphere, we can add the equality case to Theorem 3.8.

Theorem 3.9 (Combinatorial isoperimetry of balls II). Let $\Delta$ be a simplicial $(d-1)$-ball on $m+n$ vertices with $n$ vertices in the boundary and assume that $\partial \Delta \subseteq \Delta$ is a full subcomplex. Then the following inequalities hold:

(a) For every $0 \leq k \leq \frac{d}{2}$

$$
h_{k}(\Delta, \partial \Delta) \leq\left(\begin{array}{c}
m+n-d+k-1 \\
k
\end{array}\right)-\left(\begin{array}{c}
n-d+k-1 \\
k
\end{array}\right)
$$

Equality holds for some $k_{0} \leq \frac{d}{2}$ if and only if every non-face $\sigma$ of $\Delta$ of dimension $<k_{0}$ is supported in $\partial \Delta$. (b) For every $\frac{d}{2}<k \leq d$

$$
h_{k}(\Delta, \partial \Delta) \leq\left(\begin{array}{c}
m+n-1-k \\
d-k
\end{array}\right) .
$$

Equality holds for some $k_{0}>\frac{d}{2}$ if and only if $\Delta$ has no non-face $\sigma$ of dimension $<d-k_{0}$.

The bounds are tight: For every $n \geq d \geq 0$ and $m \geq 0$, there is a d-ball that attains the upper bounds for all $k$ simultaneously.

Proof of Tightness. We borrow a construction that we will see again in Section 5 and apply it to two wellchosen cyclic $(d-1)$-polytopes $C_{1}, C_{2}$ used by Matschke, Pfeifle and Pilaud [MPP11, Theorem 2.6] (compare Theorem 5.2) with $f_{0}\left(C_{1}\right)=n$ and $f_{0}\left(C_{2}\right)=m$. Let

$$
C=\operatorname{conv}\left(C_{1} \times\{0\} \cup C_{2} \times\{1\}\right) \subset \mathbb{R}^{d} \times \mathbb{R}
$$

be the Cayley polytope of $C_{1}$ and $C_{2}$. Using Theorem 2.6 of [MPP11] the Cayley polytope over $C_{1}$ and $C_{2}$ may be chosen in such a way that it has no non-face of dimension $<\frac{d}{2}-1$, and such that every non-face of dimension $\frac{d}{2}-1$ is supported in either $C_{1}$ or $C_{2}$. By construction, $C_{1}$ and $C_{2}$ are the only non-simplex faces of $C$. Let us triangulate $C_{2}$ without new vertices, and such that there are no non-faces of dimension $\leq \frac{d}{2}-1$, and let $\Delta$ be the simplicial complex obtained from $\partial C$ by deleting $C_{1}$. Then $\Delta$ is a triangulated $d$-ball with full boundary $\partial \Delta=\partial C_{1}$, and the conditions in (a) and (b) are met and hence yields an example that attains the upper bounds. 


\section{Estimating the Algebraic CONTRibution}

We discuss three techniques for bounding the $h$-vector entries $h_{k}(\Delta, \Gamma)$ based on bounds on the algebraic contribution $h^{\mathrm{alg}}(\Delta, \Gamma)$.

The first method is based on the idea of a change of presentation: We consider presentations of $\mathrm{M}[\Delta, \Gamma]$ as quotients of monomial ideals $I / J$ where $I$ is simpler in structure than $\mathrm{I}_{\Gamma}$. A particularly important candidate is the nerve ideal $\mathrm{I}_{\mathfrak{N}}$ that arises from coverings of $\Gamma$ by full subcomplexes. The nerve ideal can be analyzed in terms of nerve complex of that covering. This in particular allows us to interpolate between full and general subcomplexes $\Gamma$. As a special case, we recover Lemma 3.1.

The second method is based on a more delicate trick. It uses a formula that integrates over the $h$-numbers of subcomplexes to the $h$-vector of the total complex. We then employ a lemma of Kalai-Stanley for an upper bound on the local contributions to obtain the desired bounds. The second method has an interesting refinement that we describe in Section 4.4. In particular, we find an interesting reverse isoperimetric inequality that considerably improves on, and is substantially different from, all known bounds in the area.

Finally, we discuss the role of relative shellability, a combinatorial/geometric method that can be used to give bounds on $h$-numbers in our setting.

4.1. Estimates via change of presentation. The idea of this section is that if $M[\Delta, \Gamma]$ has a "nice" presentation as a quotient, then this presentation can be used to estimate the algebraic contribution of $\Psi=(\Delta, \Gamma)$. We will see an interesting connection to poset topology when attempting to characterize the case of equality and an application of Borsuk's Nerve Lemma (in its filtered version due to Björner).

Let $M$ be a module over $\mathbb{k}[\mathbf{x}]$. We write $M \propto I$ for a monomial ideal $I \subseteq \mathbb{k}[\mathbf{x}]$ if there is a monomial ideal $J \subseteq I$ such that $M \cong I / J$ as finely graded modules.

Lemma 4.1. Let $M \cong I / J$ be a module over $\mathbb{k}[\mathbf{x}]$ for some monomial ideals $J \subseteq I$ in $\mathbb{k}[\mathbf{x}]$. For a sequence $\Theta=\left(\theta_{1}, \ldots, \theta_{\ell}\right)$ of linear forms we then have

$$
\operatorname{dim}_{\mathbb{k}}(M / \Theta M)_{k} \leq \operatorname{dim}_{\mathbb{k}}(I / \Theta I)_{k}
$$

for all $k \geq 0$. Moreover, if tensoring with $\mathbb{k}[\mathbf{x}] / \Theta$ preserves exactness of $0 \rightarrow J \hookrightarrow I \rightarrow M \rightarrow 0$, then equality holds for some $k_{0}$ if and only if $M_{k} \cong \mathrm{I}_{k}$ for all $k \leq k_{0}$.

Proof. By assumption, we have a short exact sequence

$$
0 \longrightarrow J \longrightarrow I \longrightarrow M \longrightarrow 0
$$

The first claim follows from the fact that tensoring with $\mathbb{k}[\mathbf{x}] / \Theta \mathbb{k}[\mathbf{x}]$ is a right-exact functor and thus $I / \Theta I \rightarrow M / \Theta M$ is a (graded) surjection. Assume now that

$$
0 \longrightarrow J / \Theta J \longrightarrow I / \Theta I \longrightarrow M / \Theta M \longrightarrow 0
$$

is exact. In all nontrivial cases $\Theta$ is at best a partial l.s.o.p. for $J$. Hence, if $(J / \Theta J)_{k_{0}}=0$, then $J$ has no generators in degrees $\leq k_{0}$.

This result subsumes Lemma 3.1: If $M=\mathbb{k}[\Delta]$ is the Stanley-Reisner ring of a $(d-1)$-dimensional CohenMacaulay simplicial complex on $n$ vertices, then $M \propto I$ for $I=\mathbb{k}\left[x_{1}, \ldots, x_{n}\right]$. For a regular l.s.o.p. $\Theta$, we infer from Lemma 4.1 that

$$
h_{k}(\Delta)=\operatorname{dim}_{\mathbb{k}}(M / \Theta M)_{k} \leq \operatorname{dim}_{\mathbb{k}}(I / \Theta I)_{k}=\operatorname{dim}_{\mathbb{k}} \mathbb{k}\left[y_{1}, \ldots, y_{n-d}\right]_{k}=\left(\begin{array}{c}
n-d+k-1 \\
k
\end{array}\right) .
$$

To conclude tightness in Lemma 4.1, we need to decide whether a sequence is regular for all modules in a given exact sequence. To this end, we can use the following well-known observation. Recall that $\Theta_{m}$ is the restriction of the sequence $\Theta$ to the first $m$ elements.

Proposition 4.2. Let $R$ be any ring. Let

$$
D \rightarrow C \rightarrow B \stackrel{\varphi}{\rightarrow} A \rightarrow 0
$$


denote a exact sequence of $R$-modules, and let $\Theta=\left(\theta_{1}, \ldots, \theta_{\ell}\right)$ denote a family of elements of $R$. Assume that for every $1 \leq m \leq \ell, \varphi$ induces a surjection

$$
\left(\Theta_{m-1} B: \theta_{m}\right) / \Theta_{m-1} B \longrightarrow\left(\Theta_{m-1} A: \theta_{m}\right) / \Theta_{m-1} A .
$$

Then we have an exact sequence

$$
D / \Theta D \rightarrow C / \Theta C \rightarrow B / \Theta B \stackrel{\bar{\varphi}}{\rightarrow} A / \Theta A \rightarrow 0 .
$$

In the situation of Proposition 4.2, we also say that $\varphi$ is a $\Theta$-surjection; if the maps between the annihilator modules are even isomorphisms, then we call the map a $\Theta$-isomorphism.

Lemma 3.1 compares enumerative properties of $\Delta$ to those of the much simpler complex $\Delta_{n}$. This is possible because both are Cohen-Macaulay. For simplicial complexes, this approach suffices. In order to use a reasoning similar to Lemma 3.1 for relative complexes, we will use a cover of the subcomplex $\Gamma$ by full subcomplexes.

Definition 4.3. Let $\Delta$ be a simplicial complex. An arrangement of full complexes, full arrangement for short, is a finite collection $\mathscr{G}$ of full subcomplexes of $\Delta$.

For an arrangement $\mathscr{G}$ of complexes, the collection

$$
\mathcal{P}(\mathscr{G}):=\{\bigcap S: S \subseteq \mathscr{G}\} \cup\{\Delta\}
$$

together with the partial order given by reverse inclusion is the intersection poset of $\mathscr{G}$. This is a poset with minimal element $\hat{0}:=\Delta$ and maximal element $\hat{1}:=\bigcap \mathscr{G}$. Note that any $\Gamma \in \mathcal{P}(\mathscr{G})$ is a full subcomplex of $\Delta$. The support of $\mathscr{G}$ is the subcomplex

$$
\overline{\mathscr{G}}:=\bigcup_{\Gamma \in \mathscr{G}} \Gamma \subseteq \Delta .
$$

This covering of $\overline{\mathscr{G}}$ by full subcomplexes can be used to obtain a simple presentation of $\mathrm{M}[\Delta, \overline{\mathscr{G}}]$.

Definition 4.4. For an arrangement $\mathscr{G}$ of complexes of $\Delta$ we define the nerve ideal as the monomial ideal

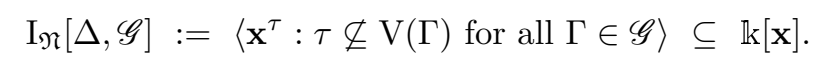

For $\Gamma \in \mathscr{G}$, the smallest simplex containing $\Gamma$ is given by $\Delta_{\mathrm{V}(\Gamma)}$. The coarse nerve of $\mathscr{G}$ is the simplicial complex

$$
\mathfrak{N}[\Delta, \mathscr{G}]:=\bigcup\left\{\Delta_{\mathrm{V}(\Gamma)}: \Gamma \in \mathscr{G}\right\} .
$$

The nerve ideal $\mathrm{I}_{\mathfrak{N}}[\Delta, \mathscr{G}]$ then is the Stanley-Reisner ideal of the coarse nerve. The connection to $(\Delta, \overline{\mathscr{G}})$ is the following.

Proposition 4.5. Let $\mathscr{G}$ be a full arrangement of $\Delta$. Then

$$
\mathrm{M}[\Delta, \overline{\mathscr{G}}] \cong\left(\mathrm{I}_{\mathfrak{N}}[\Delta, \mathscr{G}]+\mathrm{I}_{\Delta}\right) / \mathrm{I}_{\Delta} \cong \mathrm{I}_{\mathfrak{N}}[\Delta, \mathscr{G}] /\left(\mathrm{I}_{\Delta} \cap \mathrm{I}_{\mathfrak{N}}[\Delta, \mathscr{G}]\right)
$$

Proof. Let $\mathbf{x}^{\alpha}$ be a monomial and $\sigma=\operatorname{supp}\left(\mathbf{x}^{\alpha}\right)$. Then $\mathbf{x}^{\alpha}=0$ in both $\mathrm{M}[\Delta, \overline{\mathscr{G}}]$ and $\left(\mathrm{I}_{\mathfrak{N}}[\Delta, \mathscr{G}]+\mathrm{I}_{\Delta}\right) / \mathrm{I}_{\Delta}$ if $\sigma \notin \Delta$. Thus, let us assume that $\sigma \in \Delta$. Now $\mathrm{M}[\Delta, \overline{\mathscr{G}}]_{\alpha} \neq 0$ iff $\sigma \notin \overline{\mathscr{G}}$, which is the case if and only if $\sigma \notin \Gamma$ for all $\Gamma \in \mathscr{G}$. Since all subcomplexes in $\mathscr{G}$ are full, this is equivalent to $\sigma \nsubseteq \mathrm{V}(\Gamma)$ for all $\Gamma \in \mathscr{G}$. This, in turn, is equivalent to $\left(\left(\mathrm{I}_{\mathfrak{N}}[\Delta, \mathscr{G}]+\mathrm{I}_{\Delta}\right) / \mathrm{I}_{\Delta}\right)_{\alpha} \neq 0$.

For an arrangement of full subcomplexes a good relative complex to compare $\Psi=(\Delta, \Gamma)$ to is $\left(\Delta_{n}, \mathfrak{N}[\Delta, \mathscr{G}]\right)$. Proposition 4.5 and Lemma 4.1 then imply immediately:

Theorem 4.6. Let $\Delta$ be a simplicial complex, and let $\mathscr{G}$ be a full arrangement of subcomplexes. Then $\mathrm{M}=\mathrm{M}[\Delta, \overline{\mathscr{G}}] \propto \mathrm{I}_{\mathfrak{N}}=\mathrm{I}_{\mathfrak{N}}[\Delta, \mathscr{G}]$ and for every collection of linear forms $\Theta$

$$
\operatorname{dim}_{\mathbb{k}}(\mathrm{M} / \Theta \mathrm{M})_{k} \leq \operatorname{dim}_{\mathbb{k}}\left(\mathrm{I}_{\mathfrak{N}} / \Theta \mathrm{I}_{\mathfrak{N}}\right)_{k}
$$

for all $k$. If $\Theta$ is a l.s.o.p. for $M$, and the surjection $\mathrm{I}_{\mathfrak{N}} \rightarrow \mathrm{M}$ is a $\Theta$-surjection, then the following are equivalent:

(i) Equality holds for some $k_{0}$;

(ii) $\mathrm{M}_{k} \cong\left(\mathrm{I}_{\mathfrak{N}}\right)_{k}$ for all $k \leq k_{0}$; 
(iii) $\mathrm{I}_{\Delta} \cap \mathrm{I}_{\mathfrak{N}}$ is generated in degrees $>k_{0}$;

(iv) every non-face of $\Delta$ of dimension $<k_{0}$ is supported on $\mathrm{V}(\Gamma)$ for some $\Gamma \in \mathscr{G}$.

To help decide whether $I_{\mathfrak{N}} \rightarrow M$ is a $\Theta$-surjection, it is useful to keep some simple tricks in mind. For instance, if $\Theta$ is a regular sequence for $\mathrm{M}$, then $\Theta_{m-1} \mathrm{M}: \theta_{m} / \Theta_{m-1} \mathrm{M} \equiv 0$, so that $\mathrm{I}_{\mathfrak{N}} \rightarrow \mathrm{M}$ is trivially a $\Theta$-surjection. This is in particular applicable if $\mathrm{M}$ is Cohen-Macaulay.

Proposition 4.7. A l.s.o.p. $\Theta$ of length $\ell$ induces a $\Theta$-surjection $\mathrm{I}_{\mathfrak{N}} \rightarrow \mathrm{M}[\Psi]$ if the $(\ell-1)$-skeleton $\Psi^{(\ell)}$ is Cohen-Macaulay.

Proof. This follows since every l.s.o.p. $\Theta$ of length $\leq \ell$ is regular if the $(\ell-1)$-skeleton is Cohen-Macaulay by a result of Hibi, cf. [Hib91, Corollary 2.6].

For a more general criterion, we consider complexes whose skeleta are Buchsbaum.

Theorem 4.8. Assume that the $(\ell-1)$-skeleta $\left(\Delta_{\mathrm{V}(\Delta)}, \mathfrak{N}[\Delta, \mathscr{G}]\right)^{(\ell)}$ and $(\Delta, \overline{\mathscr{G}})^{(\ell)}$ are Buchsbaum, and let $\Theta$ be any l.s.o.p. of length $\ell$. Then we have a $\Theta$-surjection resp. $\Theta$-isomorphism if for every face $\sigma$ of $\Delta$, the embedding

$$
\operatorname{lk}(\sigma,(\Delta, \overline{\mathscr{G}})) \longleftrightarrow \operatorname{lk}\left(\sigma,\left(\Delta_{\mathrm{V}(\Delta)}, \mathfrak{N}[\Delta, \mathscr{G}]\right)\right)
$$

induces a surjection (resp. isomorphism) of cohomology groups up to degree $\ell-\operatorname{dim} \sigma-2$.

Proof. The basic idea is that the modules $\Theta_{m-1} I_{\mathfrak{N}}: \theta_{m} / \Theta_{m-1} I_{\mathfrak{N}}$ can be written as cokernels in short exact sequences of cohomology groups of Koszul complexes: We have

$$
0 \longrightarrow H^{m-2}\left(\Theta_{m-1} ; M\right) \longrightarrow H^{m-1}\left(\Theta_{m} ; M\right) \longrightarrow \Theta_{m-1} M: \theta_{m} / \Theta_{m-1} M \longrightarrow 0
$$

for $\mathbb{k}[x]$-modules $M$ as in Corollary 2.4. For Buchsbaum complexes, these homology modules are determined in terms of local cohomology of $I_{\mathfrak{N}}$ and $\mathrm{M}$, and by the connection between the $\mathbb{Z}^{n}$-graded Cech complex and homology of links (exploited in Hochster's formula), we conclude that if for all $\sigma \in \Delta$, the embedding (3) induces a surjection of cohomology groups up to degree $\ell-\operatorname{dim} \sigma-2$, then we also have a surjection on the level of local cohomology modules of $\mathrm{I}_{\mathfrak{N}}$ and $\mathrm{M}$ up to dimension $\ell$.

Let now $m \leq \ell$. Observe that the key to Lemma 2.2 is a quasi-isomorphism of chain complexes

$$
\tau^{\ell}\left(K^{\bullet}\left(\Theta_{m} ; \mathrm{M}\right) \otimes_{\mathbb{k}[\mathbf{x}]} C^{\bullet}(\mathrm{M})\right) \stackrel{\sim}{\longrightarrow} \tau^{\ell} K^{\bullet}\left(\Theta_{m} ; \mathrm{M}\right)
$$

in the derived category $D(\mathbb{k}[\mathbf{x}])$, cf. [Sch81, Sch82]. Here the former chain complex is a chain complex with trivial differentials, with

$$
C^{i}(\mathrm{M})= \begin{cases}H^{i}(\mathrm{M}) & \text { if } i \in\{0, \ldots, \operatorname{dim} \mathrm{M}-1\} \text { and } \\ 0 & \text { otherwise. }\end{cases}
$$

so that the complex $C^{\bullet}(\mathrm{M})$ is the exact chain complex of local cohomology modules of M, and $\tau^{\ell}$ denotes the truncation of a chain complex in degree $\ell$. Analogously, we have a quasiisomorphism

$$
\tau^{\ell}\left(K^{\bullet}\left(\Theta_{m} ; \mathrm{I}_{\mathfrak{N}}\right) \otimes_{\mathbb{k}[\mathbf{x}]} C^{\bullet}\left(\mathrm{I}_{\mathfrak{N}}\right)\right) \stackrel{\sim}{\longrightarrow} \tau^{\ell} K^{\bullet}\left(\Theta_{m} ; \mathrm{I}_{\mathfrak{N}}\right) .
$$

It follows that a surjection on the level of local cohomology modules of $\mathrm{I}_{\mathfrak{N}}$ and $\mathrm{M}$ induces a surjection on the level of Koszul cohomology.

To conclude the desired surjection of modules $\left(\Theta_{m-1} \mathrm{I}_{\mathfrak{N}}: \theta_{m}\right) / \Theta_{m-1} \mathrm{I}_{\mathfrak{N}} \longrightarrow\left(\Theta_{m-1} \mathrm{M}: \theta_{m}\right) / \Theta_{m-1} \mathrm{M}$, consider

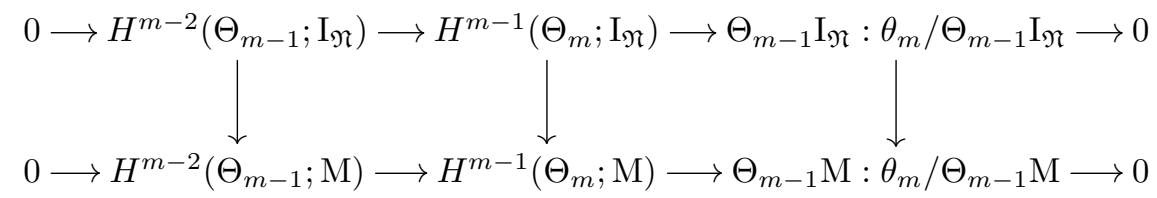

and the Snake lemma. The claim for the isomorphism follows analogously. 
This motivates us to notice a beautiful relation to Borsuk's Nerve Lemma [Bor48]: Not all full arrangements are created equal.

Let us call a full arrangement $\mathscr{G}$ in $\Delta$ an $\ell$-good cover if, for every subset $\left\{\Gamma_{1}, \ldots, \Gamma_{t}\right\}$ of $t$ elements of $\mathscr{G}$, the relative complex $\left(\Delta, \Delta \cap \bigcap_{i=1}^{t} \Gamma_{i}\right)$ is $(\ell-t)$-acyclic, that is, its homology vanishes up to dimension $\ell-t$. We call $\mathscr{G} \ell$-magnificent (w.r.t. $(\Delta, \overline{\mathscr{G}})$ ) if, for every face $\sigma$ of $\Delta$, the restriction of $\mathscr{G}$ to $\operatorname{lk}(\sigma, \Delta)$ is $(\ell-\operatorname{dim} \sigma-1)$-good. We have the following application of the Nerve Lemma (in its generalization due to Björner, cf. [Bjö03, BWW05]).

Theorem 4.9. Assume that $\mathscr{G}$ is an $\ell$-magnificent cover, and that $\left(\Delta_{\mathrm{V}(\Delta)}, \mathfrak{N}[\Delta, \mathscr{G}]\right)^{(\ell)}$ and $(\Delta, \overline{\mathscr{G}})^{(\ell)}$ are Buchsbaum. Then for every face $\sigma$ of $\Delta$, the embedding

$$
\operatorname{lk}(\sigma,(\Delta, \overline{\mathscr{G}})) \longleftrightarrow \operatorname{lk}\left(\sigma,\left(\Delta_{\mathrm{V}(\Delta)}, \mathfrak{N}[\Delta, \mathscr{G}]\right)\right)
$$

induces an isomorphism of relative homology up to dimension $\ell-\operatorname{dim} \sigma-2$, and a surjection in degree $\ell-\operatorname{dim} \sigma-1$. In particular, $\mathrm{I}_{\mathfrak{N}} \rightarrow \mathrm{M}$ is a $\Theta$-isomorphism for every l.s.o.p. $\Theta$ of length $\ell$, and a $\Theta$-surjection for every l.s.o.p. $\Theta$ of length $\ell+1$.

Example 4.10. If $\Delta$ is Cohen-Macaulay, and $\mathscr{G}$ is a collection of disjoint Cohen-Macaulay subcomplexes of $\Delta$ of the same dimension as $\Delta$, then the cover is $d$-magnificent.

We will see some more interesting examples and an application when investigating Minkowski sums of polytopes, compare also Theorem 5.5. We finally record a simple trick to compute $\operatorname{dim}_{\mathbb{k}}(\mathrm{I} / \Theta \mathrm{I})_{k}$ for Lemma 4.1 using a dual form of Schenzel's Formula.

Theorem 4.11. Let $\Gamma \subseteq \Delta_{n}$ be a simplicial complex with Stanley-Reisner ideal $\mathrm{I}_{\Gamma}$. For $m \geq \ell$ assume that the relative complex of $(m-1)$ - and $(\ell-1)$-skeleta $\left(\Delta_{n}^{(m)}, \Gamma^{(\ell)}\right)$ is Buchsbaum and let $\mathrm{M}=\mathrm{M}\left[\Delta_{n}^{(m)}, \Gamma^{(\ell)}\right]$ be the corresponding face module. If $\Theta$ is a l.s.o.p. for $\mathrm{M}$ then for all $0 \leq j \leq \ell$

$$
\operatorname{dim}_{\mathrm{k}}\left(\mathrm{I}_{\Gamma} / \Theta \mathrm{I}_{\Gamma}\right)_{j}=\left[(1-t)^{m} \mathrm{~F}\left(\mathrm{I}_{\Gamma}, t\right)\right]_{j}-\left(\begin{array}{c}
m \\
j
\end{array}\right) \sum_{i=0}^{j-1}(-1)^{j-i} \widetilde{\beta}_{i-2}\left(\Gamma^{(\ell)}\right),
$$

where $\left[(1-t)^{m} \mathrm{~F}\left(\mathrm{I}_{\Gamma}, t\right)\right]_{j}$ denotes the coefficient of $t^{j}$ in $(1-t)^{m} \mathrm{~F}\left(\mathrm{I}_{\Gamma}, t\right)$.

If $\left(\Delta_{n}^{(m)}, \Gamma^{(\ell)}\right)$ is Buchsbaum and hence locally Cohen-Macaulay, we necessarily have $\ell \leq m \leq \ell+1$ by Corollary 1.10 .

Proof. Notice that $\Delta_{n}^{(m)}$ is Cohen-Macaulay and by the long exact sequence in relative homology

$$
\widetilde{H}_{i-2}\left(\Gamma^{(\ell)}\right) \cong \widetilde{H}_{i-1}\left(\Delta_{n}^{(m)}, \Gamma^{(\ell)}\right) \text { for all } i-1<\ell-1 .
$$

Hence, by Theorem 2.5, we obtain

$$
\left[(1-t)^{m} \mathrm{~F}(\mathrm{M}, t)\right]_{j}=[\mathrm{F}(\mathrm{M} / \Theta \mathrm{M}, t)]_{j}+\left(\begin{array}{c}
m \\
j
\end{array}\right) \sum_{i=0}^{j-1}(-1)^{j-i} \widetilde{\beta}_{i-2}\left(\Gamma^{(\ell)}\right) .
$$

Passing to the $(\ell-1)$-skeleton changes the ideal $\mathrm{I}_{\Gamma}$ in degrees $>\ell$, so that $\left(\mathrm{I}_{\Gamma}\right)_{\leq \ell} \cong M_{\leq \ell}$. The formula follows.

Corollary 4.12. Let $\Gamma \subseteq \Delta_{n}$ be any simplicial complex. Assume that for $m \geq \ell$ the relative complex $\left(\Delta_{n}^{(m)}, \Gamma^{(\ell)}\right)$ is Cohen-Macaulay. If $\Theta$ is a full l.s.o.p. for $\mathrm{M}\left[\Delta_{n}^{(m)}, \Gamma^{(\ell)}\right]$, then for all $0 \leq j \leq \ell$

$$
\operatorname{dim}_{\mathrm{k}}\left(\mathrm{I}_{\Gamma} / \Theta \mathrm{I}_{\Gamma}\right)_{j}=\left[(1-t)^{m} \mathrm{~F}\left(\mathrm{I}_{\Gamma}, t\right)\right]_{j} .
$$

To summarize, we reduced the problem of bounding the $h$-numbers, or equivalently the problem of bounding $\left[(1-t)^{d} \mathrm{~F}(\mathrm{M}[\Delta, \Gamma], t)\right]_{j}$, to the problem of bounding $\left[(1-t)^{\ell} \mathrm{F}(\mathrm{I}, t)\right]_{j}$ for some Stanley-Reisner ideal with $\mathrm{M}[\Delta, \Gamma] \propto \mathrm{I}$. The full power of this approach is seen in combination with Theorem 4.6. Let us close with a simple observation that will close the cycle by computing $[\mathrm{F}(\mathrm{I}, t)]_{j}$ as a straightforward application of the inclusion-exclusion principle on the involved non-face ideals of cliques. 
Proposition 4.13. Let $\Delta$ be a pure $(d-1)$-dimensional simplicial complex, and let $\mathscr{G}$ be a full arrangement. Then

$$
\operatorname{dim}_{\mathbb{k}} \mathrm{I}_{\mathfrak{N}}[\Delta, \mathscr{G}]_{k}=\sum_{p \in \mathcal{P}(\mathscr{G})} \mu_{\mathcal{P}(\mathscr{G})}(\Delta, p)\left(\begin{array}{c}
f_{0}(p)+k-1 \\
k
\end{array}\right)
$$

where $\mu_{\mathcal{P}(\mathscr{G})}$ is the Möbius function of the intersection poset.

4.2. Arrangements of Cohen-Macaulay complexes. The estimates via change of presentation enable us to extend the results from full CM complexes to full arrangements of CM complexes.

Theorem 4.14. Let $\Delta$ be $a(d-1)$-dimensional Cohen-Macaulay complex and $\mathscr{G}=\left\{\Gamma_{1}, \ldots, \Gamma_{m}\right\}$ a full arrangement of $m$ pairwise disjoint codimension one Cohen-Macaulay subcomplexes of $\Delta$. Then for $0 \leq k \leq d$

$$
h_{k}(\Delta, \overline{\mathscr{G}}) \leq\left(\begin{array}{c}
f_{0}(\Delta)-d+k-1 \\
k
\end{array}\right)-\sum_{i=1}^{m}\left(\begin{array}{c}
f_{0}\left(\Gamma_{i}\right)-d+k-1 \\
k
\end{array}\right)+(m-1)\left(\begin{array}{c}
-d+k-1 \\
k
\end{array}\right)
$$

Equality holds for some $k_{0}$ if and only if every non-face of $\Delta$ of dimension $<k_{0}$ is supported on some $\Gamma_{i}$.

Let us write

$$
\mathbf{1}_{k \geq a}= \begin{cases}1 & \text { if } k \geq a \text { and } \\ 0 & \text { otherwise }\end{cases}
$$

Proof. Set $\Gamma:=\bar{G}=\Gamma_{1} \cup \cdots \cup \Gamma_{m}$. To begin with, we observe that for a vertex $v \in \Gamma_{i}$, we have $\operatorname{lk}(v,(\Delta, \Gamma))=$ $\operatorname{lk}\left(v,\left(\Delta, \Gamma_{i}\right)\right)$. We conclude from Corollary 1.10 that $\operatorname{lk}(\Delta, \Gamma, v)$ is Cohen-Macaulay for all vertices $v \in \Delta$. Hence, by Theorem 1.11, $\Psi=(\Delta, \Gamma)$ is Buchsbaum and $\mathrm{M}=\mathrm{M}[\Psi]$ a Buchsbaum module. We can therefore use the relative Schenzel formula (Theorem 2.5) to bound $h_{k}(\Psi)$ in terms of the topological contribution $h_{i}^{\text {top }}(\Psi)$ and the algebraic contribution $h_{i}^{\text {alg }}(\Psi)$.

The topological contribution. From the Cohen-Macaulayness of the complexes $\Gamma_{i}$ it follows that

$$
\widetilde{\beta}_{0}(\Gamma)=m-1 \text { and } \widetilde{\beta}_{d-2}(\Gamma)=\sum_{i} \widetilde{\beta}_{d-2}\left(\Gamma_{i}\right)
$$

and $\widetilde{\beta}_{i}(\Gamma)=0$ for all other $i$. The long exact sequence in relative homology

$$
\cdots \longrightarrow \widetilde{H}_{i+1}(\Delta, \Gamma) \longrightarrow \widetilde{H}_{i}(\Gamma) \longrightarrow \widetilde{H}_{i}(\Delta) \longrightarrow \widetilde{H}_{i}(\Delta, \Gamma) \longrightarrow \cdots
$$

splits into short sequences and we deduce

$$
\widetilde{\beta}_{1}(\Psi)=m-1 \text { and } \widetilde{\beta}_{d-1}(\Psi)=\widetilde{\beta}_{d-1}(\Delta)+\sum_{i} \widetilde{\beta}_{d-2}\left(\Gamma_{i}\right)
$$

and $\widetilde{\beta}_{i}(\Psi)=0$ otherwise. Hence, for $k \leq d$, the topological contribution in Theorem 2.5 is

$$
h_{k}^{\text {top }}(\Psi)=(-1)^{k-2}(m-1) \mathbf{1}_{k \geq 3}\left(\begin{array}{l}
d \\
k
\end{array}\right) .
$$

The algebraic contribution. The nerve ideal $\mathrm{I}_{\mathfrak{N}}=\mathrm{I}_{\mathfrak{N}}[\Delta, \Gamma]$ is the Stanley-Reisner ideal of the coarse nerve $\mathfrak{N}=\bigcup_{i=1}^{m} \Delta_{\mathrm{V}\left(\Gamma_{i}\right)} \subseteq \Delta_{n}$. Since each $\Gamma_{i}$ is of dimension $d-2, \mathfrak{N}$ is the disjoint union of simplices of dimension $\geq d-2$. Hence, the relative complex $\left(\Delta_{n}^{(d)}, \mathfrak{N}^{(d)}\right)$ is Cohen-Macaulay by Corollary 1.10. The homology of $\mathfrak{N}$ is concentrated in degree 0 with $\widetilde{\beta}_{0}(\mathfrak{N})=m-1$. Therefore, we obtain for a l.s.o.p. $\Theta$ for $\mathrm{M}$

$$
\begin{array}{rlrl}
h_{k}^{\text {alg }}(\Psi) & =\operatorname{dim}_{\mathbb{k}}[\mathrm{M} / \Theta \mathrm{M}]_{k} & & \\
& \leq \operatorname{dim}_{\mathbb{k}}\left(\mathrm{I}_{\mathfrak{N}} / \Theta \mathrm{I}_{\mathfrak{N}}\right)_{k} & & \text { (by Theorem 4.6) } \\
& =\left[(1-t)^{d} \mathrm{~F}\left(\mathrm{I}_{\mathfrak{N}}, t\right)\right]_{k}-\left(\begin{array}{l}
d \\
k
\end{array}\right) \sum_{i=0}^{k-1}(-1)^{k-i} \widetilde{\beta}_{i-2}(\mathfrak{N}) & \quad \text { (by Theorem 4.11) } \\
& =\sum_{p \in \mathcal{P}(\mathscr{G})} \mu_{\mathcal{P}}(\Delta, p)\left(\begin{array}{c}
f_{0}(p)-d+k-1 \\
k
\end{array}\right)-(-1)^{k-2}(m-1) \mathbf{1}_{k \geq 3}\left(\begin{array}{l}
d \\
k
\end{array}\right) . & \text { (by Proposition 4.13) }
\end{array}
$$


The intersection poset is $\mathcal{P}=\mathscr{G} \cup\{\Delta, \varnothing\}$ and hence the Möbius function is given by

$$
\mu_{\mathcal{P}(\mathscr{G})}(\Delta, p)= \begin{cases}1 & \text { if } p=\Delta \\ -1 & \text { if } p=\Gamma_{i} \text { and } \\ m-1 & \text { if } p=\varnothing\end{cases}
$$

Putting the computation of $h^{\text {top }}(\Psi)$ and the bound on $h^{\text {alg }}(\Psi)$ together yields the bound on $h_{k}(\Psi)$.

Case of Equality. Equality can hold for some $k_{0}$ if and only if it holds for the algebraic contributions. The equality is then this of Theorem 4.6.

The following result interpolates between the two extreme situations of Theorem 4.14 and the case that $\overline{\mathscr{G}}$ is itself full.

Theorem 4.15. Let $\Delta$ be a $(d-1)$-dimensional Cohen-Macaulay complex and $\mathscr{G}=\left\{\Gamma_{1}, \ldots, \Gamma_{m}\right\}$ an arrangement of $m$ pairwise disjoint, codimension one CM subcomplexes. Assume that for every subset $S$ of $[m]$ with $|S| \leq \ell, \ell>1$, the complex $\cup_{i \in S} \Gamma_{i}$ is full in $\Delta$. Then we have, for all $k \in[d]$,

$$
h_{k}\left(\Delta, \cup \Gamma_{i}\right) \leq \sum_{p \in \mathcal{P}(\mathscr{G})} \mu_{p}\left(\begin{array}{c}
f_{0}(p)-d+k-1 \\
k
\end{array}\right)+(-1)^{k-2}(m-1) \mathbf{1}_{k \geq 3}\left(\begin{array}{l}
d \\
k
\end{array}\right)+(-1)^{k+\ell+1}\left(\begin{array}{c}
m-1 \\
\ell
\end{array}\right) \mathbf{1}_{k \geq \ell+2}\left(\begin{array}{l}
d \\
k
\end{array}\right)
$$

for $\mathscr{G}=\left\{\cup_{i \in S} \Gamma_{i}: S \subseteq[m],|S| \leq \ell\right\} \cup\left\{\Delta_{0}\right\}$ and

$$
\mu_{p}=\mu_{\mathcal{P}(\mathscr{G})}(\Delta, p)=\left\{\begin{array}{cl}
1 & \text { if } p=\Delta \\
(-1)^{|S|} & \text { if } p=\cup_{i \in S} \Gamma_{i} \text { and } \\
-\sum_{j=0}^{\ell}(-1)^{j}\left(\begin{array}{c}
m \\
j
\end{array}\right) & \text { if } p=\varnothing
\end{array}\right.
$$

Proof. The proof is analogous to the proof of Theorem 4.14; the topological part is unchanged, and it remains only to estimate $\widetilde{\beta}_{i-2}(\mathfrak{N})$. But $\mathfrak{N}$ is homotopy equivalent to the $(\ell-1)$-skeleton of a simplex on $m$ vertices, so that the claim follows.

We conclude with the proofs of Theorems 3.8 and 3.9.

Proof of Theorem 3.9. By Reisner's Theorem 1.9, $\Delta$ and $(\Delta, \partial \Delta)$ are Cohen-Macaulay. Therefore, Claim (a) is a special case of Theorem 4.14 with $\mathscr{G}=\{\partial \Delta\}$. To see Claim (b), notice that by the Dehn-Sommerville relations 1.3, we have $h_{k}(\Delta, \partial \Delta)=h_{d-k}(\Delta)$ for all $0 \leq k \leq d$, and to $\Delta$ we can apply the standard upper bound theorem. Therefore, the claim follows with characterization of equality in Theorem 4.14 and Lemma 3.1.

We close with the proof for the combinatorial isoperimetric problem for manifolds.

Proof of Theorem 3.8. By Theorem 1.11, $\Psi=(M, B)$ is a Buchsbaum complex and $\mathrm{M}=\mathrm{M}[\Psi]$ a Buchsbaum module. To apply Theorem 2.5, it remains for us to bound $h_{k}(\Psi)$ in terms of the topological contribution $h_{k}^{\text {top }}(\Psi)$ and the algebraic contribution $h_{k}^{\text {alg }}(\Psi)$. The topological contribution depends only on the relative Betti numbers $(M, B)$ and hence is

$$
h_{k}^{\text {top }}(\Psi)=\left(\begin{array}{l}
d \\
k
\end{array}\right) \sum_{i=0}^{k-1}(-1)^{k-i} \widetilde{\beta}_{i-1}(\Psi) .
$$

As for the algebraic contribution: The nerve ideal $\mathrm{I}_{\mathfrak{N}}=\mathrm{I}_{\mathfrak{N}}[M, B]$ is the Stanley-Reisner ideal of the simplicial complex $\Delta_{n}, n \geq d-1$. Hence, the relative complex $\left(\Delta_{m+n}^{(d)}, \Delta_{n}^{(d)}\right)$ is Buchsbaum by Corollary 1.10 and Theorem 1.11, and in fact Cohen-Macaulay since the homology is concentrated in degree $d-1$. Therefore, by Theorems 4.6 and 4.11 and Proposition 4.13,

$$
h_{k}^{\mathrm{alg}}(\Psi) \leq\left(\begin{array}{c}
m+n-d+k-1 \\
k
\end{array}\right)-\left(\begin{array}{c}
n-d+k-1 \\
k
\end{array}\right) .
$$


4.3. Local-to-global estimates. The purpose of this section is to provide iterative inequalities of the type given in Proposition 3.2. We will provide the desired bounds for $h_{k}(\Psi)$ by combining an integration formula for multivariate formal power series with an observation of Stanley and Kalai and a careful use of the fullness property.

Lemma 4.16 (Formula for local $h$-vectors, cf. [McM70],[Swa05, Lemma 2.3]). For a pure relative simplicial complex $\Psi=(\Delta, \Gamma)$ of dimension $d-1$ on $[n]$

$$
\sum_{i=1}^{n} h_{k}(\mathrm{lk}(i, \Psi))=(k+1) h_{k+1}(\Psi)+(d-k) h_{k}(\Psi)
$$

for all $0 \leq k \leq d$.

Proof. For $\alpha=\left(\alpha_{1}, \ldots, \alpha_{n}\right) \in \mathbb{Z}^{n}$ and $1 \leq i \leq n$ let us write $\alpha \backslash i=\left(\alpha_{1}, \ldots, \alpha_{i-1}, 0, \alpha_{i+1}, \ldots, \alpha_{n}\right)$. Let us abbreviate $\frac{\partial}{\partial \mathrm{t}}:=\frac{\partial}{\partial t_{1}}+\cdots+\frac{\partial}{\partial t_{n}}$. For the fine graded Hilbert series of the face module $\mathrm{M}=\mathrm{M}[\Delta, \Gamma]$ we compute

$$
\begin{aligned}
\frac{\partial}{\partial \mathbf{t}} \mathrm{F}(\mathrm{M}, \mathbf{t}) & :=\sum_{\operatorname{supp}(\alpha) \in \Psi} \frac{\partial}{\partial \mathbf{t}} \mathbf{t}^{\alpha}=\sum_{i=1}^{n} \sum_{\operatorname{supp}(\alpha) \in \Psi} \frac{\partial}{\partial t_{i}} \mathbf{t}^{\alpha} \\
& =\sum_{i=1}^{n} \sum_{\operatorname{supp}(\alpha) \in \operatorname{st}(i, \Psi)} \alpha_{i} t_{i}^{\alpha_{i}-1} \mathbf{t}^{\alpha \backslash i}=\sum_{i=1}^{n} \frac{1}{\left(1-t_{i}\right)^{2}} \sum_{\operatorname{supp}(\alpha) \in \operatorname{lk}(i, \Psi)} \mathbf{t}^{\alpha \backslash i} .
\end{aligned}
$$

If we now specialize $t_{1}=\cdots=t_{n}=t$, we obtain

$$
\frac{\mathrm{d}}{\mathrm{d} t} \mathrm{~F}(\mathrm{M}, t)=\frac{1}{(1-t)^{2}} \sum_{i=1}^{n} \mathrm{~F}(\mathrm{M}[\mathrm{lk}(i, \Psi)], t)=\sum_{i=1}^{n} \frac{\sum_{k=0}^{d-1} h_{k}(\mathrm{lk}(i, \Psi)) t^{k}}{(1-t)^{d+1}}
$$

where $\mathrm{F}(\mathrm{M}, t)$ is the coarse Hilbert series. On the other hand we can directly compute the derivative of $\mathrm{F}(\mathrm{M}, t)$ as

$$
\begin{aligned}
\frac{\mathrm{d}}{\mathrm{d} t} \mathrm{~F}(\mathrm{M}, t) & =\frac{\mathrm{d}}{\mathrm{d} t} \frac{\left.\sum_{k=0}^{d} h_{k}(\Psi)\right) t^{k}}{(1-t)^{d}}=\frac{\left.\sum_{k=0}^{d} k h_{k}(\Psi)\right) t^{k-1}}{(1-t)^{d}}+\frac{\left.\sum_{k=0}^{d} d h_{k}(\Psi)\right) t^{k}}{(1-t)^{d+1}} \\
& =\frac{\sum_{k=0}^{d-1}\left((k+1) h_{k+1}(\Psi)+(d-k) h_{k}(\Psi)\right) t^{k}}{(1-t)^{d+1}}
\end{aligned}
$$

To bound $h_{k}(\operatorname{st}(v, \Psi))$, we need a relative version of a simple lemma of Stanley [Sta93] and Kalai [Kal91].

Lemma 4.17. Let $\Psi=(\Delta, \Gamma)$ be a relative complex of dimension $d-1$ on vertex set $[n]$. Let $\Delta^{\prime} \subseteq \Delta$ be any subcomplex of $\Delta$, and set $\Gamma^{\prime}=\Delta^{\prime} \cap \Gamma$ and $\Psi^{\prime}=\left(\Delta^{\prime}, \Gamma^{\prime}\right)$. Then, for every $k$,

$$
\operatorname{dim}_{\mathrm{k}}\left(\mathrm{M}\left[\Psi^{\prime}\right] / \Theta \mathrm{M}\left[\Psi^{\prime}\right]\right)_{k} \leq \operatorname{dim}_{\mathrm{k}}(\mathrm{M}[\Psi] / \Theta \mathrm{M}[\Psi])_{k} .
$$

Proof. This follows immediately if we consider $\mathrm{M}\left[\Psi^{\prime}\right]$ as an $\mathbb{k}[\mathbf{x}]$-module: By right-exactness of the tensor product, we have a degree preserving surjection $\mathrm{M}[\Psi] / \Theta \mathrm{M}[\Psi] \longrightarrow \mathrm{M}\left[\Psi^{\prime}\right] / \Theta \mathrm{M}\left[\Psi^{\prime}\right]$.

The last ingredient is a property for vertex stars of full subcomplexes.

Lemma 4.18. Let $\Gamma \subseteq \Delta$ be a pair of simplicial complexes. Then $\Gamma$ is full in $\Delta$ if and only if $\operatorname{st}(v, \Gamma)=$ $\operatorname{st}(v, \Delta) \cap \Gamma$ for all $v \in \mathrm{V}(\Gamma)$.

For a relative complex $\Psi=(\Delta, \Gamma)$ and a vertex $v \in \Delta$, let us write $\widetilde{\operatorname{st}}(v, \Psi):=(\operatorname{st}(v, \Delta), \operatorname{st}(v, \Delta) \cap \Gamma)$.

Example 4.19. Let $\Psi=(\Delta, \Gamma)$ be a relative complex such that both $\Delta$ and $\Gamma$ are Cohen-Macaulay of the same dimension and $\Gamma$ is full in $\Delta$. By Lemmas 4.16 and 1.2

$$
\sum_{v \in \Delta} h_{k}(\operatorname{st}(v, \Psi))=(k+1) h_{k+1}(\Psi)+(d-k) h_{k}(\Psi) .
$$


Since $\Gamma$ is full, Lemma 4.18 yields $\operatorname{st}(v, \Psi)=\widetilde{\operatorname{st}}(v, \Psi)$ for all vertices $v \in \Gamma$. Therefore, for a l.s.o.p. $\Theta$ and for every vertex $v \in \Gamma$

$$
\begin{aligned}
h_{k}(\operatorname{st}(v, \Psi)) & =h_{k}(\widetilde{\operatorname{st}}(v, \Psi)) \\
& =\operatorname{dim}_{\mathbb{k}}(\mathrm{M}[\widetilde{\operatorname{st}}(v, \Psi)] / \Theta \mathrm{M}[\widetilde{\operatorname{st}}(v, \Psi)])_{k} \\
& \leq \operatorname{dim}_{\mathbb{k}}(\mathrm{M}[\Psi] / \Theta \mathrm{M}[\Psi])_{k} \\
& =h_{k}(\Psi)
\end{aligned}
$$

(by Cohen-Macaulayness).

If $v \notin \Gamma$, the reasoning becomes a little more difficult as $\operatorname{st}(v, \Psi)$ not necessarily coincides with $\widetilde{\operatorname{st}}(v, \Psi)$ any more. However, we can simply estimate

$$
\begin{aligned}
h_{k}(\operatorname{st}(v, \Psi)) & =h_{k}(\operatorname{st}(v, \Delta)) \\
& =\operatorname{dim}_{\mathbb{k}}(\mathrm{M}[\operatorname{st}(v, \Delta)] / \Theta \mathrm{M}[\operatorname{st}(v, \Delta)])_{k} \\
& \leq \operatorname{dim}_{\mathbb{k}}(\mathrm{M}[\Delta] / \Theta \mathrm{M}[\Delta])_{k} \\
& =h_{k}(\Delta) \\
& =h_{k}(\Psi)+h_{k}(\Gamma)
\end{aligned}
$$

(by Cohen-Macaulayness)

(by Lemma 4.17)

(by Cohen-Macaulayness)

(by linearity of the h-vector).

These inequalities, for the special case of simplicial polytopes were the key to McMullen's proof of the UBT for polytopes. Integrating these inequalities over all vertices and using Lemma 4.16, we obtain

$$
(k+1) h_{k+1}(\Psi) \leq\left(f_{0}(\Delta)-d+k\right) h_{k}(\Psi)+f_{0}(\Psi) h_{k}(\Gamma) .
$$

To handle situations with $\operatorname{dim} \Gamma<\operatorname{dim} \Delta$, let us define for $\ell \geq 0$

$$
h_{i}^{\langle\ell\rangle}(\Psi):=\left[(1-t)^{\ell} \mathrm{F}(\mathrm{M}[\Psi], t)\right]_{i}=\sum_{k=0}^{i}(-1)^{i-k}\left(\begin{array}{c}
\ell-k \\
\ell-i
\end{array}\right) f_{k-1}(\Psi) .
$$

Comparing this with the definition of $h$-vectors in Section 1, we see that $h_{i}^{\langle d\rangle}(\Psi)=h_{i}(\Psi)$ for $d=\operatorname{dim} \Psi+1$. Also, for an arrangement $\mathscr{G}$ and a vertex $v \in \Delta$ we set $\mathscr{G}(v):=\{\Gamma \in \mathscr{G}: v \in \Gamma\}$ and consequently $\overline{\mathscr{G}}(v):=\bigcup_{\Gamma \in \mathscr{G}(v)} \Gamma$. We call the pair $(\Delta, \mathscr{G})$ universally Buchsbaum of dimension $d-1$ if for every vertex $v$ of the $(d-1)$-complex $\Delta$, the relative complex $(\Delta, \bar{G}(v))$ is Buchsbaum of dimension $(d-1)$.

Lemma 4.20. Let $(\Delta, \mathscr{G})$ be universally Buchsbaum of dimension $d-1$, where $\mathscr{G}$ is some full arrangement of subcomplexes of $\Delta$. Let $v$ be any vertex of $\Delta$. Then

$$
h_{k}(\operatorname{lk}(v, \Delta), \operatorname{lk}(v, \overline{\mathscr{G}})) \leq h_{k}(\Delta, \overline{\mathscr{G}}(v))-h_{k}^{\mathrm{top}}(\Delta, \overline{\mathscr{G}}(v)) .
$$

Proof. Let $v \in \Delta$ be a vertex and let us write $\Psi(v)=(\Delta, \bar{G}(v))$. With a l.s.o.p. $\Theta$ we deduce

$$
\begin{array}{rlr}
h_{k}(\operatorname{lk}(v, \Delta), \operatorname{lk}(v, \overline{\mathscr{G}})) & =h_{k}(\operatorname{lk}(v, \Psi(v)) \\
& =h_{k}(\operatorname{st}(v, \Psi(v)) & \\
& =h_{k}(\widetilde{\operatorname{st}}(v, \Psi(v)) & \text { (Lemma 1.2) } \\
& =\operatorname{dim}_{\mathbb{k}}(\mathrm{M}[\widetilde{\operatorname{st}}(v, \Psi(v))] / \Theta \mathrm{M}[\widetilde{\operatorname{st}}(v, \Psi(v))])_{k} & \text { (using fullness) } \\
& \leq \operatorname{dim}_{\mathbb{k}}(\mathrm{M}[\Psi(v)] / \Theta \mathrm{M}[\Psi(v)])_{k} & \text { (since } \widetilde{\operatorname{st}}(v, \Psi(v)) \text { is CM) }
\end{array}
$$

By Theorem 2.5, the last expression equals $h_{k}(\Delta, \overline{\mathscr{G}}(v))-h_{k}^{\text {top }}(\Delta, \overline{\mathscr{G}}(v))$.

Summing equation (5) over all vertices of $\Delta$ and using Lemma 4.16 as in Example 4.19, we obtain the following result.

Theorem 4.21. Let $\Psi=(\Delta, \overline{\mathscr{G}})$ be a relative complex of dimension $d-1$ where $\mathscr{G}$ is a full arrangement. If $(\Delta, \mathscr{G})$ is universally Buchsbaum, then

$$
(k+1) h_{k+1}(\Psi) \leq\left(f_{0}(\Delta)-d+k\right) h_{k}(\Psi)+\sum_{v \in \Delta}\left(h_{k}^{\langle d\rangle}(\overline{\mathscr{G}}, \overline{\mathscr{G}}(v))-h_{k}^{\mathrm{top}}(\Delta, \overline{\mathscr{G}}(v))\right) .
$$


The results are somewhat simpler if the pair $(\Delta, \mathscr{G})$ is universally Cohen-Macaulay of dimension $d-1$, i.e., $(\Delta, \bar{G}(v))$ is Cohen-Macaulay of dimension $d-1$ for every vertex $v \in \Delta$. This also means that the topological terms in Theorem 4.21 vanish.

Corollary 4.22. Let $(\Delta, \mathscr{G})$ be universally Cohen-Macaulay of dimension $d-1$ where $\mathscr{G}$ is a full arrangement of $\Delta$.

(1) For every vertex $v \in \Delta$

$$
h_{k}(\mathrm{l}(v, \Delta), \operatorname{lk}(v, \overline{\mathscr{G}})) \leq h_{k}(\Delta, \overline{\mathscr{G}}(v))=h_{k}(\Delta, \overline{\mathscr{G}})+h_{k}^{\langle d\rangle}(\overline{\mathscr{G}}, \overline{\mathscr{G}}(v))
$$

Equality holds up to some $k_{0}$ if and only if for every $\sigma$ of $(\Delta, \mathscr{G}(v))$ of dimension $<k_{0}$, the simplex $\sigma * v$ is a face of $\Delta$.

(2) Moreover, we have

$$
(k+1) h_{k+1}(\Psi) \leq\left(f_{0}(\Delta)-d+k\right) h_{k}(\Psi)+\sum_{v \in \Delta} h_{k}^{\langle d\rangle}(\overline{\mathscr{G}}, \overline{\mathscr{G}}(v)) .
$$

Equality holds if and only if it holds for all $v$ in (1).

Proof. (1) and (2) are Lemma 4.20 and Theorem 4.21 for universally CM pairs. It remains to characterize the equality cases. Recall that the inclusion $\operatorname{st}(v, \Delta) \cap \overline{\mathscr{G}}(v) \subseteq \overline{\mathscr{G}}(v)$ induces a degree-preserving surjection

$$
\varphi_{v}: \mathrm{M}[\Psi(v)]=\mathrm{I}_{\overline{\mathscr{G}}(v)} / \mathrm{I}_{\Delta} \longrightarrow \mathrm{I}_{\overline{\mathscr{G}}(v)} / \mathrm{I}_{\mathrm{st}(v, \Delta)}=\mathrm{M}[\widetilde{\mathrm{st}}(v, \Psi(v))] .
$$

Since $\mathrm{M}[\widetilde{\operatorname{st}}(v, \Psi(v))]$ is $\mathrm{CM}$, for a l.s.o.p. $\Theta$ for $\mathrm{M}[\Psi(v)]$ we get a short exact sequence

$$
0 \longrightarrow \operatorname{ker}\left(\varphi_{v}\right) / \Theta \operatorname{ker}\left(\varphi_{v}\right) \longrightarrow \mathrm{M}[\Psi(v)] / \Theta \mathrm{M}[\Psi(v)] \longrightarrow \mathrm{M}[\widetilde{\operatorname{st}}(v, \Psi(v))] / \Theta \mathrm{M}[\widetilde{\operatorname{st}}(v, \Psi(v))] \longrightarrow 0
$$

Therefore, equality holds if and only if the surjection $\varphi_{v}$ is an isomorphism if and only if

$$
\left(\operatorname{ker}\left(\varphi_{v}\right) / \Theta \operatorname{ker}\left(\varphi_{v}\right)\right)_{\leq k_{0}}=(\mathrm{M}[\Delta, \overline{\mathscr{G}}(v) \cup \operatorname{st}(v, \Delta)] / \Theta \mathrm{M}[\Delta, \overline{\mathscr{G}}(v) \cup \operatorname{st}(v, \Delta)])_{\leq k_{0}}=0
$$

which is only the case if the face module $\mathrm{M}[\Delta, \overline{\mathscr{G}}(v) \cup \operatorname{st}(v, \Delta)]$ is generated in degree $>k_{0}$.

Remark 4.23. The equality cases in Theorem 4.21 are a bit harder to characterize; one can use Proposition 4.2 and Theorem 4.8.

4.4. A reverse isoperimetric inequality. We can use the philosophy of Lemma 4.17 in yet another way to provide upper bounds on algebraic $h$-numbers by replacing Lemma 4.17 with a stronger inequality. The results, even though they require more work, yield inequalities stronger than the ones provided in Section 4.3. For simplicity, we focus on the Cohen-Macaulay case and leave the general case to the interested reader.

Let $\Psi=(\Delta, \Gamma)$ be a relative complex. A relative subcomplex of $\Psi$ is a relative complex $\Psi^{\prime}=\left(\Delta^{\prime}, \Gamma^{\prime}\right)$ with $\Delta^{\prime} \subseteq \Delta$ and $\Gamma^{\prime} \subseteq \Gamma$. The pair $\left(\Psi, \Psi^{\prime}\right)$ is again a relative complex with face module

$$
\mathrm{M}\left[\Psi, \Psi^{\prime}\right]:=\operatorname{ker}\left(\mathrm{M}[\Psi] \rightarrow \mathrm{M}\left[\Psi^{\prime}\right]\right) \cong \mathrm{M}\left[\Delta, \Gamma \cup \Delta^{\prime}\right] .
$$

We say that $\Psi^{\prime}$ is a full relative subcomplex if $\Delta^{\prime} \subseteq \Delta$ is full.

Theorem 4.24. Let $\Psi$ be a $(d-1)$-dimensional Cohen-Macaulay relative complex and $\Psi^{\prime}$ a codimension one Cohen-Macaulay full relative subcomplex. Then

$$
h_{k}\left(\Psi, \Psi^{\prime}\right) \geq h_{k-1}\left(\Psi^{\prime}\right)
$$

for all $k$.

Proof. Let $\theta_{1}, \ldots, \theta_{d}$ be a l.s.o.p. for $\mathrm{M}=\mathrm{M}[\Psi]$ such that $\Theta=\left(\theta_{1}, \ldots, \theta_{d-1}\right)$ is a l.s.o.p. for $\mathrm{M}^{\prime}=\mathrm{M}\left[\Psi^{\prime}\right]$ and $\theta_{d}$ is a linear form $\theta_{d} \in \mathbb{k}$-span $\left\{x_{v}: v \notin \mathrm{V}\left(\Delta^{\prime}\right)\right\}$; this can be done as $\Delta^{\prime}$ is full in $\Delta$ and of codimension one, so that every facet of $\Delta$ contains at least one vertex not in $\Delta^{\prime}$, compare also [Sta96, Section III.9].

Consider the injective map $\widetilde{\varphi}: \mathrm{M} / \Theta \mathrm{M} \rightarrow \mathrm{M} / \Theta \mathrm{M}$ given by multiplication by $\theta_{d}$. Now $\theta_{d} \mathrm{M} \subseteq \mathrm{M}\left[\Psi, \Psi^{\prime}\right]$ by choice of $\theta_{d}$ and hence, we get a homogeneous map $\varphi: \mathrm{M} / \Theta \mathrm{M} \rightarrow \mathrm{M}\left[\Psi, \Psi^{\prime}\right] /\left(\Theta, \theta_{d}\right) \mathrm{M}\left[\Psi, \Psi^{\prime}\right]$ of degree one induced by the multiplication with $\theta_{d}$. Again by the regularity of $\theta_{d}$ and using the fullness property, the kernel of $\varphi$ is given by $\operatorname{ker} \varphi=\mathrm{M}\left[\Psi, \Psi^{\prime}\right] / \Theta \mathrm{M}\left[\Psi, \Psi^{\prime}\right]$. Factoring out the kernel we get an injection

$$
\bar{\varphi}: \mathrm{M}^{\prime} / \Theta \mathrm{M}^{\prime} \longleftrightarrow \mathrm{M}\left[\Psi, \Psi^{\prime}\right] /\left(\Theta, \theta_{d}\right) \mathrm{M}\left[\Psi, \Psi^{\prime}\right]
$$


Since $\bar{\varphi}$ is homogeneous of degree one, we obtain

$$
h_{k-1}\left(\Psi^{\prime}\right)=\operatorname{dim}_{\mathbb{k}}\left(\mathrm{M}^{\prime} / \Theta \mathrm{M}^{\prime}\right)_{k-1} \leq \operatorname{dim}_{\mathbb{k}}\left(\mathrm{M}\left[\Psi, \Psi^{\prime}\right] /\left(\Theta, \theta_{d}\right) \mathrm{M}\left[\Psi, \Psi^{\prime}\right]\right)_{k}=h_{k}\left(\Psi, \Psi^{\prime}\right) .
$$

A simple application of this inequality yields a reverse isoperimetric inequality.

Corollary 4.25. Let $\Delta$ be a simplicial ball and assume that $\partial \Delta$ is full in $\Delta$, then

$$
h_{k}(\Delta, \partial \Delta) \geq h_{k-1}(\partial \Delta)
$$

for all $k$.

In the same situation, Lemma 4.17 only yields $h_{k}(\Delta, \partial \Delta) \geq 0$ which also follows easily since $(\Delta, \partial \Delta)$ is Cohen-Macaulay. This almost is a Lefschetz-type result that characterizes primitive Betti numbers, compare also [Sta96]. We refer to [Adi15] for related applications towards a quantitative lower bound theorem, and also Remark 5.18 for a small application.

4.5. Relations to relative shellability. The estimates of Section 4.3 are reminiscent of McMullen's approach to $h$-vectors and the Upper Bound Theorem via shellings. In this section we want to put our techniques into perspective via the notion of relative shellability. The results presented here are not essential for the following sections, but provide a combinatorial viewpoint.

Let $\Psi=(\Delta, \Gamma)$ be a pure relative complex of dimension $d-1$ and let $F \in \Delta \backslash \Gamma$ be a facet. The deletion $\Psi^{\prime}=\Psi-F:=(\Delta-F, \Gamma)$ is a step in a relative shelling if $\Psi^{\prime} \cap F$ is pure of codimension one. A relative complex is shellable if there is sequence of shelling steps to the relative complex $(\Gamma, \Gamma)$. If $\Gamma=\varnothing$, then this is the classical notion of shelling of simplicial complexes. Relative shellings where introduced by Stanley [Sta87] and further developed in [AB12]. Shellability has proven to be an invaluable tool in topological combinatorics. The basis for our situation is the following result due to Kind-Kleinschmidt [KK79] and Stanley [Sta96].

Proposition 4.26. A shellable relative complex is Cohen-Macaulay over any ground field.

In particular, the $h$-vector of a relative complex can be read off a shelling.

Proposition 4.27. Let $\Psi^{\prime}=\Psi-F$ be a shelling step and let $\sigma$ be the unique minimal face in $2^{F} \backslash \Psi^{\prime}$. Then $h_{k}(\Psi)=h_{k}\left(\Psi^{\prime}\right)+1$ for $k=|\sigma|$ and $h_{k}(\Psi)=h_{k}\left(\Psi^{\prime}\right)$ otherwise.

Let us revisit the situation of Example 4.19 from the perspective of relative shellings: We call $\Psi=(\Delta, \Gamma)$ universally shellable if for every vertex $v$ of $\Delta$ there is a shelling of $\Psi$ that removes $\operatorname{st}(v, \Psi)$ first. For a universally shellable complex $\Psi$ such that $\Gamma$ is full, the arguments of [McM70] (see also [Zie95, Section 8.4]) yield once again

$$
(k+1) h_{k+1}(\Psi) \leq\left(f_{0}(\Delta)-d+k\right) h_{k}(\Psi)+f_{0}(\Psi) h_{k}(\Gamma) .
$$

This is sufficient to provide a solution to the upper bound problem for universally shellable relative complexes in the sense of Lemma 3.1; see also Theorem 3.9. The challenge, of course, is to show that a given relative complex is shellable, that is, to exhibit an actual shelling. For this, one can use a variety of methods from poset theory [Bjö80], geometry [BM71], and tools such as Alexander duality and gluing theorems for relative shellings, cf. [AB12].

\section{The Upper Bound Theorem for Minkowski Sums}

We now come to our main application of relative Stanley-Reisner theory: A tight upper bound theorem for Minkowski sums of polytopes. In analogy to the classical UBT, the class of polytopes that maximize the number of $k$-faces is rather special and we devote the first section to their definition and the statement of results. The proofs are rather intricate and we illustrate the main ideas in the case of two summands $P_{1}+P_{2}$ in Section 5.2 which recovers the results of [KT11] with a simple argument.

The transition from Minkowski sums to relative simplicial complexes is via the Cayley polytope and the (relative) Cayley complex, whose definition and properties are presented in Subsection 5.3. In particular, the Cayley complex allows us to introduce the notion of an $h$-vector for special families of simplicial polytopes and reduce the upper bound problem to one on $h$-vectors. The general scheme for the proof is then similar to that 
of the UBT for polytopes: We will prove sharp upper bounds for the 'first half' of the $h$-vector (Section 5.4). For the 'second half' of the $h$-vector we prove Dehn-Sommerville-type relations in Section 5.5. Unfortunately, this formula does not express $h_{k}$ of the second half as positive linear combinations of such from the first half, so that we need a further strengthening of the bounds provided in Section 5.6. We finally conclude the Upper Bound Theorem for Minkowski sums (Theorem 5.19). While some statements in this section are general, we focus in this section on Minkowski sums of pure collections, i.e., Minkowski sums of polytopes in $\mathbb{R}^{d}$ with at least $d+1$ vertices each. We discuss the nonpure case in Section 6 .

5.1. Minkowski-neighborly polytopes and main results. Let us recall the setup for the Minkowski upper bound problem. For given $m, d \geq 1$ and $\mathbf{n}=\left(n_{1}, \ldots, n_{m}\right) \in \mathbb{Z}_{\geq d+1}^{m}$, we seek to find tight upper bounds on

$$
f_{k}\left(P_{1}+P_{2}+\cdots+P_{m}\right)
$$

for polytopes $P_{1}, \ldots, P_{m}$ such that $f_{0}\left(P_{i}\right)=n_{i}$ for all $i=1, \ldots, m$. We shall focus here on pure families, that is, families where each of the summands has at least $d+1$ vertices. To ease the notational burden, let us write $P_{[m]}:=\left(P_{1}, \ldots, P_{m}\right)$ and $f_{k}\left(P_{[m]}\right)=\left(f_{k}\left(P_{1}\right), \ldots, f_{k}\left(P_{m}\right)\right)$. We also abbreviate $\left|P_{[m]}\right|:=P_{1}+P_{2}+\cdots+P_{m}$ for the Minkowski sum of a family. We extend these notions to subfamilies $P_{S}=\left(P_{i}: i \in S\right)$ for $S \subseteq[m]$.

As for the UBT, we can make certain genericity assumptions. Recall that every face $F$ of $\left|P_{[m]}\right|$ can be written as $F=F_{1}+\cdots+F_{m}=\left|F_{[m]}\right|$ where $F_{i} \subseteq P_{i}$ are unique nonempty faces. It follows that

$$
\operatorname{dim} F \leq \operatorname{dim} F_{1}+\cdots+\operatorname{dim} F_{m} .
$$

We call the polytopes $P_{[m]}$ in relatively general position if equality holds in (6) for all proper faces $F \subsetneq\left|P_{[m]}\right|$. Similar to the situation of the UBT for polytopes and spheres, it is possible to reduce the UBPM to simplicial polytopes in relatively general position by a simple perturbation; compare [FW10, Theorem 1]. We need a notion similar to neighborliness of polytopes that will describe the polytopes attaining the upper bound.

Definition 5.1. Let $P_{[m]}=\left(P_{1}, \ldots, P_{m}\right)$ be a collection of polytopes in $\mathbb{R}^{d}$. Then $P_{[m]}$ is Minkowski $(k, \ell)$-neighborly for $k \geq 0$ if for every subset $J \subseteq[m]$ of cardinality $\ell$, and for any choice of vertices $\varnothing \neq S_{j} \subseteq \mathrm{V}\left(P_{j}\right)$ with $j \in J$ such that

$$
\sum_{j \in J}\left|S_{j}\right| \leq k+|J|-1
$$

the polytope $\sum_{j \in J} \operatorname{conv}\left(S_{j}\right)$ is a simplex of $\left|P_{J}\right|$.

For $\ell=1$, this recovers the definition of $k$-neighborly polytopes. For $\ell=m$, the number of $k$-faces in a Minkowski $(k, m)$-neighborly family, if it exists, satisfies

$$
f_{k}\left(\left|P_{[m]}\right|\right)=\sum_{\substack{\alpha \in \mathbb{Z}_{\geq 1}^{m} \\
|\alpha|=m+k}} \prod_{i=1}^{m}\left(\begin{array}{c}
f_{0}\left(P_{i}\right) \\
\alpha_{i}
\end{array}\right)
$$

which is the trivial upper bound for face numbers of Minkowski sums. The following theorem characterizes Minkowski neighborly polytopes and generalizes the standard properties of neighborly polytopes.

Theorem 5.2. Let $m, d \geq 1$ be fixed.

(i) There is no pure Minkowski $(k, \ell)$-neighborly family $P_{[m]}$ in $\mathbb{R}^{d}$ for $k+\ell-1>\frac{d+\ell-1}{2}$.

(ii) For all $\mathbf{n} \in \mathbb{Z}_{\geq d+1}^{m}$ there is a family $P_{[m]}$ in $\mathbb{R}^{d}$ with $f_{0}\left(P_{[m]}\right)=\mathbf{n}$ that is Minkowski $(k, \ell)$-neighborly for all $\ell \leq m$ and $\ell-1 \leq k+\ell-1 \leq\left\lfloor\frac{d+\ell-1}{2}\right\rfloor$.

The first claim is a straightforward consequence of Radon's Theorem once we phrase the UBPM in the language of Cayley polytopes; cf. Proposition 5.6. It suffices to prove the assertion for $\ell=m$, the general case of the assertion is a straightforward corollary.

As for Theorem 5.2(ii), the construction is provided by Theorem 2.6 in [MPP11]. The constructions are based on cyclic polytopes and generalize those of [KT11, KKT15]. 
Theorem 5.2 suggests the following notion: A family $P_{[m]}$ of polytopes is called Minkowski neighborly if $P_{[m]}$ is Minkowski $(k, \ell)$-neighborly for all $\ell \leq m$ and $\ell-1 \leq k+\ell-1<\left\lfloor\frac{d+\ell-1}{2}\right\rfloor$. As in the case of the UBT for spheres, the face numbers of Minkowski neighborly polytopes only depend on $m, d$ and $f_{0}\left(P_{[m]}\right)$.

Proposition 5.3. If $P_{[m]}, P_{[m]}^{\prime}$ are two Minkowski neighborly families of $m$ simplicial d-polytopes with $f_{0}\left(P_{[m]}\right)=f_{0}\left(P_{[m]}^{\prime}\right)$, then $f_{k}\left(\left|P_{[m]}\right|\right)=f_{k}\left(\left|P_{[m]}^{\prime}\right|\right)$ for all $0 \leq k \leq d$.

This result will be a simple consequence of the Dehn-Sommerville relations for Cayley complexes developed in Section 5.5; cf. Corollary 5.15. Unless $\sum_{i} f_{0}\left(P_{i}\right) \leq d+m$, it is not true that Minkowski neighborly families of simplicial polytopes in relative general position have combinatorially equivalent Minkowski sums, so that the combinatorial types of such Minkowski sums remain to be understood; instead, Proposition 5.3 allows us to study the face numbers.

With the help of Proposition 5.3, we define $\operatorname{nb}_{k}(d, m, \mathbf{n}):=f_{k}\left(\left|\mathrm{Nb}_{[m]}\right|\right)$ for $m, d \geq 1$ and $\mathbf{n} \in \mathbb{Z}_{\geq d+1}^{m}$, where $\mathrm{Nb}_{[m]}$ is any Minkowski neighborly family of $m$ simplicial $d$-polytopes in $\mathbb{R}^{d}$ with $\mathbf{n}=f_{0}\left(\mathrm{Nb}_{[m]}\right)$.

Theorem 5.4 (Upper Bound Theorem for Minkowski sums). Let $m, d \geq 1$ and $\mathbf{n} \in \mathbb{Z}_{\geq d+1}^{m}$. If $P_{[m]}=$ $\left(P_{1}, \ldots, P_{m}\right)$ is a pure family of $m$ polytopes in $\mathbb{R}^{d}$ with $f_{0}\left(P_{[m]}\right)=\mathbf{n}$, then

$$
f_{k}\left(\left|P_{[m]}\right|\right) \leq \operatorname{nb}_{k}(d, m, \mathbf{n})
$$

for all $k=0, \ldots, d-1$. Moreover, the family $P_{[m]}$ is Minkowski neighborly if and only if equality holds for some $k_{0}, k_{0}+1 \geq \frac{d+2 m-2}{2}$.

Unfortunately, closed formulas for $\mathrm{nb}_{k}(d, m, \mathbf{n})$ are rather involved, even for small $k$. As in the case of the UBT for polytopes/spheres, upper bounds are best expressed in terms of $h$-numbers. We introduce $h$-numbers for simplicial families in relatively general position in the next section and give a rigorous treatment in Section 5.3.

5.2. Minkowski sums of two polytopes. In this section we illustrate the general proof strategy along the case of two summands. Let $P_{[2]}=\left(P_{1}, P_{2}\right)$ be two simplicial $d$-dimensional polytopes in $\mathbb{R}^{d}$ in relatively general position with $f_{0}\left(P_{[2]}\right)=\left(n_{1}, n_{2}\right)$. We seek to find the maximum possible $f_{k}\left(\left|P_{[2]}\right|\right)=f_{k}\left(P_{1}+P_{2}\right)$ for any fixed choice of $k$. Let us define the Cayley polytope of $P_{[2]}$ as the $(d+1)$-dimensional polytope

$$
C=\operatorname{Cay}\left(P_{1}, P_{2}\right):=\operatorname{conv}\left(P_{1} \times\{0\} \cup P_{2} \times\{1\}\right) \subseteq \mathbb{R}^{d} \times \mathbb{R}
$$

as sketched in Figure 5.1.

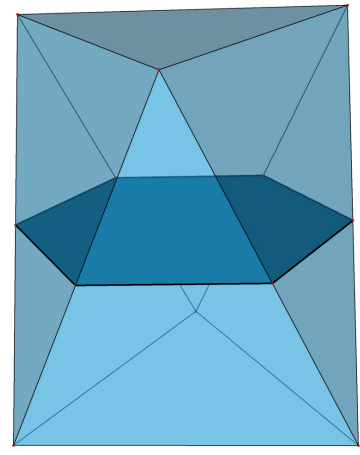

Figure 5.1. Cayley polytope of two triangles and the middle section.

The Cayley polytope has the favorable property that for $L=\mathbb{R}^{d} \times\left\{\frac{1}{2}\right\}$

$$
C \cap L \cong P_{1}+P_{2}
$$

where the isomorphism is affine. As the intersection of $L$ with faces of $C$ is transverse, we infer

$$
f_{k}\left(P_{1}+P_{2}\right)=f_{k}(C \cap L)=f_{k+1}(C)-f_{k+1}\left(P_{1}\right)-f_{k+1}\left(P_{2}\right)
$$


for $k=0, \ldots, d-1$. By assumption on $P_{[2]}$, the only proper faces of $C$ which are possibly not simplices are $P_{1}$ and $P_{2}$ and we define $\Delta:=\partial C \backslash\left\{P_{1}, P_{2}\right\}$ as the simplicial complex spanned by all proper faces different from $P_{1}$ and $P_{2}$. Observe that the boundary complexes $\partial P_{1}, \partial P_{2}$ are disjoint subcomplexes of $\Delta$ and we define $\Gamma:=\partial P_{1} \cup \partial P_{2}$. For the relative simplicial complex $\Psi=(\Delta, \Gamma)$, we record

$$
f_{k}\left(P_{1}+P_{2}\right)=f_{k+1}(\Psi)=f_{k+1}(\Delta)-f_{k+1}(\Gamma)
$$

for all $k=0, \ldots, d-1$. For later perspective, $\Delta$ is called the Cayley complex, and $(\Delta, \Gamma)$ is the relative Cayley complex.

We can now appeal to Observation 1.1 to reduce the task to bounding $h_{k}(\Psi)$ instead. Hence, we define $h_{k}\left(P_{[2]}\right):=h_{k}(\Delta, \Gamma)$ for $i=0, \ldots, d$. This setup now fits into the scheme of a relative upper bound problem. Using the developed techniques of relative Stanley-Reisner theory we can resolve this upper bound problem which recovers the the main theorem of Karavelas and Tzanaki [KT11, Theorem 18].

Theorem 5.5 (UBT for two summands). Let $P_{[2]}=\left(P_{1}, P_{2}\right)$ be two simplicial d-polytopes in relatively general position with $n_{1}$ and $n_{2}$ vertices, respectively. Then

$$
h_{k+1}\left(P_{[2]}\right) \leq\left(\begin{array}{c}
n_{1}+n_{2}-d+k-1 \\
k+1
\end{array}\right)-\left(\begin{array}{c}
n_{1}-d+k-1 \\
k+1
\end{array}\right)-\left(\begin{array}{c}
n_{2}-d+k-1 \\
k+1
\end{array}\right)+(-1)^{k+1}\left(\begin{array}{l}
d+1 \\
k+1
\end{array}\right)
$$

for $k+1 \leq\left\lfloor\frac{d+1}{2}\right\rfloor$ and

$$
h_{k+1}\left(P_{[2]}\right) \leq\left(\begin{array}{c}
n_{1}+n_{2}-k-2 \\
d-k
\end{array}\right)+(-1)^{k+1}\left(\begin{array}{l}
d+1 \\
k+1
\end{array}\right)
$$

for $k+1>\left\lfloor\frac{d+1}{2}\right\rfloor$. Equality holds for all $k$ simultaneously if and only if $P_{[2]}$ is Minkowski neighborly.

Proof. The complex $\Delta^{\prime}:=\Delta \cup P_{1} \cup P_{2}$ is a $d$-sphere and hence Cohen-Macaulay. In particular $\bar{G}=\left\{\partial P_{1}, \partial P_{2}\right\}$ is, up to excision, a full arrangement of disjoint codimension one CM subcomplexes of $\Delta^{\prime}$. For the first inequality is provided by Theorem 4.14 .

For the second inequality, we use the Dehn-Sommerville relations for relative complexes (Lemma 1.3) together with the fact that $(\Delta, \overline{\mathscr{G}})$ is weakly Eulerian. Finally, we observe that the full arrangement $\overline{\mathscr{G}}$ is $d$-magnificent in the sense of Theorem 4.9 (see example Example 4.10). It now follows with Theorems 4.6 and 4.9 that tightness in the inequalities implies the desired neighborliness.

5.3. Cayley polytopes and Cayley complexes. The geometric construction of the previous section is easily generalized to higher dimensions. For a family $P_{[m]}=\left(P_{1}, \ldots, P_{m}\right)$ of $m$ polytopes in $\mathbb{R}^{d}$, we define the Cayley polytope as

$$
\operatorname{Cay}\left(P_{[m]}\right):=\operatorname{conv}\left(\bigcup_{i=1}^{m} P_{i} \times e_{i}\right) \subseteq \mathbb{R}^{d} \times \mathbb{R}^{m} .
$$

The coordinate projection $\mathbb{R}^{d} \times \mathbb{R}^{m} \rightarrow \mathbb{R}^{m}$ restricts to a linear projection

$$
\pi: \operatorname{Cay}\left(P_{[m]}\right) \longrightarrow \Delta_{m-1}=\operatorname{conv}\left\{e_{1}, \ldots, e_{m}\right\}
$$

of the Cayley polytope to the (geometric) standard $(m-1)$-simplex. It is easy to see that for $\lambda=\left(\lambda_{1}, \ldots, \lambda_{m}\right) \in$ $\Delta_{m-1}$, we have

$$
\pi^{-1}(\lambda) \cong \lambda_{1} P_{1}+\cdots+\lambda_{m} P_{m} .
$$

In particular, for any $\lambda \in$ relint $\Delta_{m-1}, \pi^{-1}(\lambda)$ is combinatorially equivalent (and even normally equivalent, cf. [Zie95, Section 7]) to $P_{1}+\cdots+P_{m}$. Let us denote by $\Delta_{J}=\operatorname{conv}\left\{e_{i}: i \in J\right\}$ the faces of $\Delta_{m-1}$ for the various subsets $J \subseteq[m]$. Cayley polytopes are an indispensable tool in the study of Minkowski sums, cf. [dLRS10]. For nonempty faces $F_{i} \subseteq P_{i}$ for $i=1, \ldots, m$

$$
F_{1}+\cdots+F_{m} \subseteq\left|P_{[m]}\right| \text { is a face } \Longleftrightarrow \operatorname{Cay}\left(F_{1}, \ldots, F_{m}\right) \subseteq \operatorname{Cay}\left(P_{1}, \ldots, P_{m}\right) \text { is a face; }
$$

see [dLRS10, Observation 9.2.1]. Together with the next result, this correspondence yields a simple proof of Theorem 5.2(i). 
Proposition 5.6. Let $P_{[m]}$ be a family of $m$ polytopes in $\mathbb{R}^{d}$. If $\sum_{i=1}^{m} f_{0}\left(P_{i}\right)>d+m$, then there exist a choice of vertices $\varnothing \neq S_{i} \subseteq \mathrm{V}\left(P_{i}\right)$ for $i=1, \ldots, m$ with

$$
\sum_{i=1}^{m}\left|S_{i}\right| \leq\left\lfloor\frac{d+m+1}{2}\right\rfloor
$$

such that $\operatorname{Cay}\left(\operatorname{conv}\left(S_{1}\right), \ldots, \operatorname{conv}\left(S_{m}\right)\right)$ is not a face of $\operatorname{Cay}\left(P_{[m]}\right)$.

Proof. Let $M$ be any choice of $d+m+1$ vertices of the $(d+m-1)$-dimensional Cayley polytope in $\mathbb{R}^{d+m-1}$. By Radon's theorem, $M$ may be partitioned into two sets $M_{1}, M_{2}$, whose convex hulls intersect. Without loss of generality, we may assume that $M_{1}$ is the smaller of the two, so that $\left|M_{1}\right| \leq\left\lfloor\frac{d+m+1}{2}\right\rfloor$. Hence $\operatorname{conv}\left(M_{1}\right)$ is the desired non-face of Cay $\left(P_{[m]}\right)$.

The following simple proposition summarizes the most important properties of the Cayley polytope. For proofs and more information see [dLRS10]. An illustration of the Cayley polytope for three summands is given in Figure 5.2.

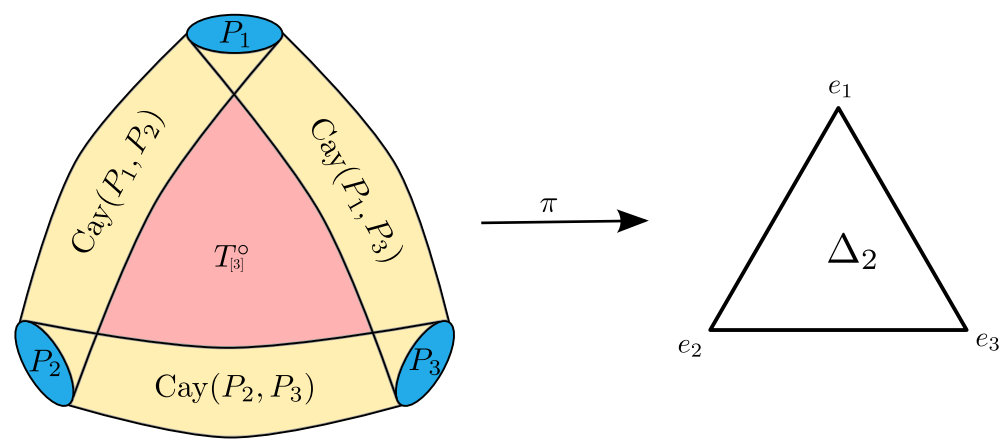

Figure 5.2. An illustration of the Cayley polytope for $P_{[3]}=\left(P_{1}, P_{2}, P_{3}\right)$ including the projection to the simplex.

Proposition 5.7. Let Cay $\left(P_{[m]}\right)$ be the Cayley polytope associated to $P_{[m]}=\left(P_{1}, \ldots, P_{m}\right)$, and let $\pi$ : Cay $\left(P_{[m]}\right) \rightarrow \Delta_{m-1}$ denote the projection of Cayley polytopes (7).

(i) For $\lambda \in$ relint $\Delta_{J}, \pi^{-1}(\lambda)$ is combinatorially equivalent to $\sum_{i \in J} P_{i}$.

(ii) For any $J \subseteq[m]$

$$
\pi^{-1}\left(\Delta_{J}\right) \cong \operatorname{Cay}\left(P_{J}\right)
$$

(iii) If all polytopes $P_{i}$ are of the same dimension $d$, i.e., if $P_{[m]}$ is pure, then

$$
\operatorname{dim} \operatorname{Cay}\left(P_{J}\right)=d+|J|-1
$$

for all $J \subseteq[m]$.

(iv) If $P_{[m]}$ is a family of simplicial polytopes in relatively general position, then the only non-simplex faces of $\operatorname{Cay}\left(P_{[m]}\right)$ are $\operatorname{Cay}\left(P_{J}\right)$ for all $\varnothing \neq J \subseteq[m]$.

The proposition suggests that the boundary of the Cayley polytope $\operatorname{Cay}\left(P_{[m]}\right)$ is stratified along the facial structure of the $(m-1)$-simplex. We define the Cayley complex $\mathrm{T}_{[m]}=\mathrm{T}\left(P_{[m]}\right)$ as the closure of $\pi^{-1}\left(\right.$ relint $\left.\Delta_{m-1}\right) \cap \partial \operatorname{Cay}\left(P_{[m]}\right)$.

Then a family $P_{[m]}$ of simplicial polytopes is in relatively general position if and only if $\mathrm{T}_{[m]}$ is a simplicial complex. For a subset $S \subseteq[m]$, let us write $\mathrm{T}_{S}:=\mathrm{T}\left(P_{S}\right)$, and $\mathrm{T}_{\varnothing}=\Delta_{0}$. It is easy to see that the boundary of $\mathrm{T}_{[m]}$ is covered by the Cayley complexes $\mathrm{T}_{J}$ for $J \subsetneq[m]$ and we define the Cayley arrangement as $\mathscr{T}:=\left\{\mathrm{T}_{[m] \backslash j}: j \in[m]\right\}$.

Example 5.8. Consider $P_{[3]}=\left(P_{1}, P_{2}, P_{3}\right)$ a family of three distinct pentagons. Then $\overline{\mathscr{T}}$ is a two-dimensional torus, cf. Figure 5.3, which is glued from the Cayley complexes for Cay $\left(P_{i}, P_{j}\right), 1 \leq i<j \leq 3$, and $\mathrm{T}_{[3]}$ is the complementary three-dimensional torus in the Cayley polytope Cay $\left(\mathrm{T}_{[3]}\right)$. 


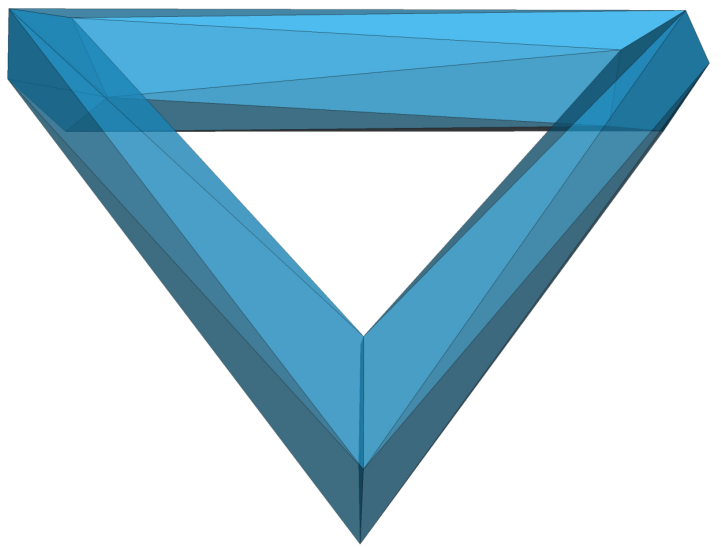

Figure 5.3. Boundary of the Cayley complex for $P_{[3]}=\left(P_{1}, P_{2}, P_{3}\right)$ being three general position pentagons in $\mathbb{R}^{2}$. The Cayley complex $\mathrm{T}_{[3]}$ is a solid torus in the three-sphere $\partial \operatorname{Cay}\left(\mathrm{T}_{[3]}\right)$

Finally, we define the relative Cayley complex as

$$
\mathrm{T}_{[m]}^{\circ}:=\left(\mathrm{T}_{[m]}, \overline{\mathscr{T}}=\bigcup_{i} \mathrm{~T}_{[m] \backslash i}\right)
$$

and consequently $\mathrm{T}_{\varnothing}^{\circ}=\varnothing$. For $S \subseteq[m]$, we define the restrictions $\mathrm{T}_{S}, \mathscr{T}_{S}$, and $\mathrm{T}_{S}^{\circ}$ analogously.

To apply our techniques, it remains to see that the topological properties of the Cayley complex are wellbehaved.

Proposition 5.9. Let $P_{[m]}=\left(P_{1}, \ldots, P_{m}\right)$ be a family of simplicial d-polytopes in relatively general position. Let $\mathrm{T}_{[m]}=\mathrm{T}\left(P_{[m]}\right)$ be the corresponding Cayley complex and $\mathscr{T}$ the Cayley arrangement.

(i) For $0 \leq k \leq d-1$

$$
f_{k+m-1}\left(\mathrm{~T}_{[m]}^{\circ}\right)=f_{k}\left(\left|P_{[m]}\right|\right) .
$$

(ii) $\mathscr{T}$ is an arrangement of full subcomplexes of $\mathrm{T}_{[\mathrm{m}]}$.

(iii) $\mathrm{T}_{[m]}^{\circ}=\left(\mathrm{T}_{[m]}, \overline{\mathscr{T}}\right)$ is relative Buchsbaum. In fact, $\mathrm{T}_{[m]}$ is a manifold, and $\overline{\mathscr{T}}$ is its boundary.

(iv) $\left(\mathrm{T}_{[m]}, \mathscr{T}\right)$ is universally Cohen-Macaulay.

(v) We have $\beta_{i}\left(\mathrm{~T}_{[m]}^{\circ}\right)=\mathbf{1}_{i=m-1}+\mathbf{1}_{i=d+m-2}$.

Proof. The relative complex $\mathrm{T}_{[m]}^{\circ}$ is exactly the set of faces of $\operatorname{Cay}\left(P_{[m]}\right)$ for which the intersection with $\pi^{-1}(\lambda)$ is nonempty for any $\lambda \in$ relint $\Delta_{m-1}$. For any such $\lambda$ the intersection of $\pi^{-1}(\lambda)$ is normally equivalent to $\left|P_{[m]}\right|$ (i.e., their normal fans coincide). Hence, the $f$-vector of $\mathrm{T}_{[m]}^{\circ}$ is the shifted $f$-vector of $\left|P_{[m]}\right|$ which proves (i).

For (ii) note that $\mathrm{T}_{S}$ is the subcomplex of $\mathrm{T}_{[m]}$ induced by the vertices $\mathrm{V}\left(P_{i}\right), i \in S$.

Let $W=\pi^{-1}\left(\partial \Delta_{m-1}\right) \subsetneq \partial \operatorname{Cay}\left(P_{[m]}\right)$ be the shadow boundary, which can be seen as a subset of $\mathbb{R}^{d} \times \partial \Delta_{m-1}$. The fibers $\pi^{-1}(x), x \in \partial \Delta_{m-1}$ are convex and of dimension $d$; hence $W$ is a full-dimensional submanifold of $\mathbb{R}^{d} \times \partial \Delta_{m-1}$ that collapses to $\partial \Delta_{m-1} \cong S^{m-1}$. It follows in particular that $W$ is in fact homeomorphic to $B_{d} \times S^{m-1}$ where $B_{d}$ is a $d$-ball.

For $(\mathrm{v})$, we can use excision to compute $\widetilde{H}_{\bullet}\left(\mathrm{T}_{[m]}, \bar{T}\right) \cong \widetilde{H}_{\bullet}\left(\partial \operatorname{Cay}\left(P_{[m]}\right), W\right)$. The same argument applied to (relative) links then shows (iii) and (iv).

So, for a proof of Theorem 5.4 it is sufficient to find tight upper bounds on the $h$-vector of the Cayley complex. To emphasize the relation to $P_{[m]}$, we define the $h$-vector of a simplicial family in relatively general position as

$$
h_{*}\left(P_{[m]}\right):=h_{*}\left(\mathrm{~T}_{[m]}^{\circ}\right) .
$$


In particular, $\mathrm{T}_{\{i\}}=\partial P_{i}$. For two summands, the relative Cayley complex is a cylinder over a sphere relative to its boundary; cf. Section 5.2.

5.4. Initial terms of the $h$-vector. In the proof of the UBT, it is only necessary to find tight upper bounds on $h_{k}$ for $k \leq\left\lfloor\frac{d}{2}\right\rfloor$ and let the Dehn-Sommerville equations take care of the rest. In this section we find bounds for $h_{k}$ for $k \leq \frac{d-m+1}{2}$. For higher $k$, we will also employ suitably generalized versions of the Dehn-Sommerville equations which we treat in the next section. In contrast to the case of spheres, we will need bounds on $g$-vectors (and more).

Theorem 5.10. Let $P_{[m]}=\left(P_{1}, \ldots, P_{m}\right)$ be a family of simplicial d-polytopes in $\mathbb{R}^{d}$ with Cayley complex $\mathrm{T}_{[m]}=\mathrm{T}\left(P_{[m]}\right)$. Then we have

$$
(k+m) g_{k+m}\left(\mathrm{~T}_{[m]}^{\circ}\right) \leq\left(f_{0}\left(\mathrm{~T}_{[m]}\right)-d-m\right) h_{k+m-1}\left(\mathrm{~T}_{[m]}^{\circ}\right)+\sum_{i=1}^{m} f_{0}\left(\mathrm{~T}_{\{i\}}\right) g_{k+m-1}\left(\mathrm{~T}_{[m] \backslash\{i\}}^{\circ}\right) .
$$

We have

$$
(i+|S|) g_{i+|S|}\left(\mathrm{T}_{S}^{\circ}\right)=\left(f_{0}\left(\mathrm{~T}_{S}\right)-d-|S|\right) h_{i+|S|-1}\left(\mathrm{~T}_{S}^{\circ}\right)+\sum_{i \in S} f_{0}\left(\mathrm{~T}_{\{i\}}\right) g_{i+|S|-1}\left(\mathrm{~T}_{S \backslash\{i\}}^{\circ}\right)
$$

for all $i \leq k_{0}$ and $S \subseteq[m]$ if and only if all non-faces of $\mathrm{T}_{S}$ of dimension $<k_{0}+|S|$ are supported in some $\mathrm{V}\left(\mathrm{T}_{R}\right), R \subsetneq S$.

Proof. By Proposition 5.9(iv), the pair $\left(\mathrm{T}_{[m]}, \mathscr{T}\right)$ is universally Cohen-Macaulay. By Corollary 4.22(2), we conclude at once that

$$
(k+m) h_{k+m}\left(\mathrm{~T}_{[m]}^{\circ}\right) \leq\left(f_{0}\left(\mathrm{~T}_{[m]}\right)-d+k\right) h_{k+m-1}\left(\mathrm{~T}_{[m]}^{\circ}\right)+\sum_{i=1}^{m} f_{0}\left(\mathrm{~T}_{\{i\}}\right) g_{k+m-1}\left(\mathrm{~T}_{[m] \backslash\{i\}}^{\circ}\right) .
$$

The desired inequality follows by subtracting $(k+m) h_{k+m-1}\left(\mathrm{~T}_{[m]}^{\circ}\right)$ on both sides. The characterization for the case of equality follows with iterative application of the characterization in Corollary 4.22(2).

Theorem 5.10 is the key to the UBTM. An alternative, geometric proof can be given rather elegantly using relative shellability: It is a consequence of the work of Bruggesser-Mani [BM71], Proposition 4.27 and Alexander duality of shellings provided in [AB12]. This program has been implemented to some extent in [KT15].

The theorem directly enables us to give (tight) upper bounds on small $h$-entries. The following corollary is a direct consequence of Theorem 5.10. We present an alternative, direct proof by change of presentation (see Section 4.1).

Corollary 5.11. Let $P_{[m]}=\left(P_{1}, \ldots, P_{m}\right)$ be a family of $m$ simplicial d-polytopes in relatively general position and let $\mathrm{T}_{[m]}^{\circ}$ be the corresponding relative Cayley complex. Then for all $-m+1 \leq k \leq d$

$$
h_{k+m-1}\left(\mathrm{~T}_{[m]}^{\circ}\right) \leq \sum_{S \subseteq[m]}(-1)^{m-|S|}\left(\begin{array}{c}
f_{0}\left(\mathrm{~T}_{S}\right)-d+k-1 \\
k+m-1
\end{array}\right),
$$

Equality holds for some $k_{0}+m-1$ if and only if all non-faces of $\mathrm{T}_{[m]}$ of dimension $<k_{0}+m-1$ are supported in some $\mathrm{V}\left(\mathrm{T}_{S}\right), S \subsetneq[m]$.

Proof. By Proposition 5.9(iii), the relative complex $\mathrm{T}_{[m]}^{\circ}=\left(\mathrm{T}_{[m]}, \overline{\mathscr{T}}\right)$ is Buchsbaum and thus we can apply Theorem 2.5.

For the topological contribution, we use Proposition 5.9(v) to infer that all Betti numbers are zero except for $\widetilde{\beta}_{m-1}\left(\mathrm{~T}_{[m]}^{\circ}\right)=1$ and $\widetilde{\beta}_{d+m-2}\left(\mathrm{~T}_{[m]}^{\circ}\right)=1$. Hence, for $k \geq 2$

$$
h_{k+m-1}^{\text {top }}\left(\mathrm{T}_{[m]}^{\circ}\right)=\left(\begin{array}{c}
d+m-1 \\
d-k
\end{array}\right) \sum_{i=0}^{k+m-2}(-1)^{k+m-1-i} \widetilde{\beta}_{i-1}\left(\mathrm{~T}_{[m]}^{\circ}\right)=\mathbf{1}_{k \geq 2}(-1)^{k-1}\left(\begin{array}{c}
d+m-1 \\
d-k
\end{array}\right) \text {. }
$$


For the algebraic component $h_{k+m-1}^{\text {alg }}\left(\mathrm{T}_{[m]}^{\circ}\right)$, recall from Proposition 5.9(ii) that $\mathscr{T}$ is an arrangement of full subcomplexes of $\mathrm{T}_{[m]}$. Hence, for $\mathrm{M}=\mathrm{M}\left[\mathrm{T}_{[m]}^{\circ}, \bar{T}\right]$, nerve ideal $\mathrm{I}_{\mathfrak{N}}=\mathrm{I}_{\mathfrak{N}}\left[\mathrm{T}_{[m]}, \bar{T}\right]$, and a l.s.o.p. $\Theta$ of length $\ell=d+m-1$ we obtain for $-m+1 \leq k \leq d$

$$
h_{k+m-1}^{\mathrm{alg}}\left(\mathrm{T}_{[m]}, \overline{\mathscr{T}}\right)=\operatorname{dim}_{\mathbb{k}}(\mathrm{M} / \Theta \mathrm{M})_{k+m-1} \leq \operatorname{dim}_{\mathbb{k}}\left(\mathrm{I}_{\mathfrak{N}} / \Theta \mathrm{I}_{\mathfrak{N}}\right)_{k+m-1}
$$

by Theorem 4.6. Now, upon closer examination, we notice that $\mathrm{I}_{\mathfrak{N}}$ is generated by squarefree monomials corresponding to subsets $\tau \subseteq \bigcup_{i} \mathrm{~V}\left(P_{i} \times e_{i}\right)$ such that $\tau \cap \mathrm{V}\left(P_{i} \times e_{i}\right) \neq \varnothing$ for all $i$. Hence, $\mathrm{I}_{\mathfrak{N}}$ is generated in degree $m$ by $\prod_{i} f_{0}\left(P_{i}\right)$ minimal generators. That is, the coarse nerve $\mathfrak{N}$ with Stanley-Reisner ideal $I_{\mathfrak{N}}$ is

$$
\mathfrak{N}=\bigcup_{S \subsetneq[m]} *{ }_{i \in S} \Delta_{\mathrm{V}\left(P_{i} \times e_{i}\right)}
$$

While $\mathfrak{N}$ is in general not Buchsbaum, its $(d+m-2)$-skeleton is. In particular $\mathfrak{N}^{(d+m-1)}$ has homology only in dimensions $d+m-2$ and $m-2$ and $\widetilde{\beta}_{m-2}\left(\mathfrak{N}^{(d+m-2)}\right)=1$. By Corollary 1.10 and Theorem 1.11, the relative complex $\left(\Delta_{[n]}^{(d+m-1)}, \mathfrak{N}^{(d+m-1)}\right)$ is Buchsbaum. Moreover, the intersection poset $\mathcal{P}=\mathcal{P}(\mathscr{T})$ of the arrangement coincides with the dual to the face poset of $\Delta_{[m]}$ and hence

$$
\mu_{\mathcal{P}}\left(\mathrm{T}_{[m]}, \mathrm{T}_{S}\right)=(-1)^{m-|S|}
$$

for all $S \subseteq[m]$. We can now use Theorem 4.11 and Proposition 4.13 to evaluate

$$
\operatorname{dim}_{\mathrm{k}}\left(\mathrm{I}_{\mathfrak{N}} / \Theta \mathrm{I}_{\mathfrak{N}}\right)_{k+m-1}=\sum_{S \subseteq[m]}(-1)^{m-|S|}\left(\begin{array}{c}
f_{0}\left(\mathrm{~T}_{S}\right)-d+k-1 \\
k+m-1
\end{array}\right)-(-1)^{k-1} \mathbf{1}_{k \geq 2}\left(\begin{array}{c}
d+m-1 \\
d-k
\end{array}\right) .
$$

Consider the cover $\widehat{\mathscr{T}}:=\left\{\mathrm{T}_{\bar{i}}:=\mathrm{T}_{[m] \backslash\{i\}}: i \in[m]\right\}$ of $\partial \mathrm{T}_{[m]}=\overline{\mathscr{T}}$. For tightness, notice that for every strict subset $R \subsetneq[m]$ of $\widehat{\mathscr{T}},\left(\mathrm{T}_{[m]}, \mathrm{T}_{[m]} \cap \bigcap_{i \in R} \mathrm{~T}_{\bar{i}}\right)$ is acyclic, that is, its Betti numbers are trivial. On the other hand,

$$
\left(\mathrm{T}_{[m]}, \mathrm{T}_{[m]} \cap \bigcap_{i \in[m]} \mathrm{T}_{\bar{i}}\right)=\left(\mathrm{T}_{[m]},\{\varnothing\}\right)
$$

has only one nontrivial homology group, that in dimension $d-1$. Hence, $\widehat{\mathscr{T}}$ is $(d+m-2)$-magnificent w.r.t. $\left(\mathrm{T}_{[\mathrm{m}]}, \overline{\mathscr{T}}\right)$ in the sense of Theorem 4.9. Tightness follows with Theorem 4.6 and Theorem 4.8.

5.5. The Dehn-Sommerville formula and other linear relations. Let us give some linear relations among the $h$-vectors of our particular simplicial complexes. To give Dehn-Sommerville-type relations among the entries of the $h$-vector of the relative Cayley complex, it will prove useful to renormalize the $h$-vector to

$$
\widetilde{h}_{k+m-1}\left(\mathrm{~T}_{[m]}^{\circ}\right):=h_{k+m-1}\left(\mathrm{~T}_{[m]}^{\circ}\right)+(-1)^{k}\left(\begin{array}{l}
d+m-1 \\
k+m-1
\end{array}\right)
$$

and

$$
\widetilde{g}_{k+m-1}\left(\mathrm{~T}_{[m]}^{\circ}\right):=\widetilde{h}_{k+m-1}\left(\mathrm{~T}_{[m]}^{\circ}\right)-\widetilde{h}_{k+m-2}\left(\mathrm{~T}_{[m]}^{\circ}\right) .
$$

On a purely enumerative level, this corresponds to setting the number of empty faces of $\mathrm{T}_{[m]}^{\circ}$ to

$$
\tilde{f}_{-1}\left(\mathrm{~T}_{[m]}^{\circ}\right)=\widetilde{h}_{0}\left(\mathrm{~T}_{[m]}^{\circ}\right)=(-1)^{m-1}=-\mu_{\mathcal{P}\left(\mathscr{T}_{[m]}\right)}\left(\mathrm{T}_{[m]}, \varnothing\right) .
$$

With this, we can compute $\widetilde{h}$ from $\left(\widetilde{f}_{-1}, f_{0}, \cdots\right)$ as usual and rewrite Corollary 5.11 as

$$
\widetilde{h}_{k+m-1}\left(\mathrm{~T}_{[m]}^{\circ}\right) \leq \sum_{\varnothing \neq S \subseteq[m]}(-1)^{m-|S|}\left(\begin{array}{c}
f_{0}\left(\mathrm{~T}_{S}\right)-d+k-1 \\
k+m-1
\end{array}\right) .
$$

An important ingredient to our approach is, once again, Dehn-Sommerville duality.

Lemma 5.12. Let $P_{[m]}$ be a pure collection of $m$ polytopes in $\mathbb{R}^{d}, d \geq 1$, such that the Cayley complex $\mathrm{T}\left(P_{1}, \ldots, P_{m}\right)$ is simplicial. Then, for all $-m+1 \leq k \leq d$, we have

$$
h_{d-k}\left(\mathrm{~T}_{[m]}\right)=\widetilde{h}_{k+m-1}\left(\mathrm{~T}_{[m]}^{\circ}\right)=h_{k+m-1}\left(\mathrm{~T}_{[m]}^{\circ}\right)+(-1)^{k}\left(\begin{array}{c}
d+m-1 \\
d-k
\end{array}\right) .
$$


Proof. By Proposition 5.9, the complexes $\mathrm{T}_{[m]}^{\circ}$ and $\mathrm{T}_{[m]}$ are homology manifolds. Moreover, $\widetilde{\beta}_{m-1}\left(\mathrm{~T}_{[m]}^{\circ}\right)=1$ and $\widetilde{\beta}_{d+m-2}\left(\mathrm{~T}_{[m]}^{\circ}\right)=1$, but $\widetilde{\beta}_{i}\left(\mathrm{~T}_{[m]}^{\circ}\right) \equiv 0$ otherwise. The assertion now follows by Lemma 1.3.

This allows us to translate from bounds on $h_{*}\left(\mathrm{~T}_{[m]}\right)$ to bounds on $h_{*}\left(\mathrm{~T}_{[m]}^{\circ}\right)$, and vice versa. Let us define

$$
\widetilde{g}_{k+m-1}^{\langle\ell\rangle}(\cdot):=\sum_{i=0}^{\ell}(-1)^{i}\left(\begin{array}{l}
\ell \\
i
\end{array}\right) \widetilde{h}_{k+m-1-i}(\cdot) \text { and } g_{k+m-1}^{\langle\ell\rangle}(\cdot):=\sum_{i=0}^{\ell}(-1)^{i}\left(\begin{array}{l}
\ell \\
i
\end{array}\right) h_{k+m-1-i}(\cdot) .
$$

With this, we have the following elementary relations.

Proposition 5.13. For $\mathrm{T}_{[m]}$ as above, any $\ell \geq 0$, and any $1 \leq s \leq m$, we have

$$
h_{k+m-1}\left(\mathrm{~T}_{[m]}\right)=\sum_{S \subseteq[m]} \widetilde{g}_{k+m-1}^{\langle m-|S|\rangle}\left(\mathrm{T}_{S}^{\circ}\right) .
$$

Proof. From the stratification of the Cayley complex $\mathrm{T}_{[m]}$ into the open Cayley complexes $\mathrm{T}_{S}^{\circ}, S \subseteq[m]$, it follows by linearity of $h$-vector that

$$
h_{k+m-1}\left(\mathrm{~T}_{[m]}\right)=\sum_{S \subseteq[m]} g_{k+m-1}^{\langle m-|S|\rangle}\left(\mathrm{T}_{S}^{\circ}\right) .
$$

Now, observe that the binomial correction terms, when passing from $g_{*}$ to $\widetilde{g}_{*}$, cancel out in the sum (9) since $\sum_{S \subseteq[m]}-1^{S}=0$, so that

$$
\sum_{S \subseteq[m]} g_{k+m-1}^{\langle m-|S|\rangle}\left(\mathrm{T}_{S}^{\circ}\right)=\sum_{S \subseteq[m]} \widetilde{g}_{k+m-1}^{\langle m-|S|\rangle}\left(\mathrm{T}_{S}^{\circ}\right)
$$

To visualize Proposition 5.13, note that every $g$-vector entry $\widetilde{g}_{k+m-1}^{\langle m-|S|\rangle}\left(\mathrm{T}_{S}^{\circ}\right)$ can be written as a sum of $h$-numbers $\widetilde{h}_{i}^{\langle m-|S|\rangle}\left(\mathrm{T}_{S}^{\circ}\right)$. Hence, we can encode the formula of Proposition 5.13 by recording the coefficients of these $h$-numbers in the following table:

\begin{tabular}{c|cccccc} 
& $|S|=m$ & $m-1$ & $m-2$ & $m-3$ & $m-4$ & $\cdots$ \\
\hline$k+m-1$ & 1 & 1 & 1 & 1 & 1 & $\cdots$ \\
$k+m-2$ & 0 & -1 & -2 & -3 & -4 & $\cdots$ \\
$k+m-3$ & 0 & 0 & 1 & 3 & 6 & $\cdots$ \\
$k+m-4$ & 0 & 0 & 0 & -1 & -4 & $\cdots$ \\
$\vdots$ & $\vdots$ & $\vdots$ & $\vdots$ & $\vdots$ & $\vdots$ & $\ddots$
\end{tabular}

Table 5.1. Visualization of Proposition 5.13, recording the coefficients for $h$-numbers.

For $k, m, d \in \mathbb{Z}$, let us now define $\mathrm{c}: \mathbb{Z}^{3} \rightarrow \mathbb{Z}$ by

$$
\mathrm{c}^{\prime}(k, m, d):=2 k+2 m-1-d \quad \Longleftrightarrow \quad k+m-1=\frac{d+\mathrm{c}^{\prime}(k, m, d)-1}{2},
$$

and

$$
\mathrm{c}(k, m, d):=\min \left\{m, \max \left\{\mathrm{c}^{\prime}(k, m, d), 1\right\}\right\} .
$$

Let us furthermore denote the covering relation by $\prec$, i.e., for $R, S \subseteq[m]$ with $R \subseteq S$ we write $R \prec S$ to denote the fact that there is no set $Q$ with $R \subsetneq Q \subsetneq S$. 
Lemma 5.14. For $\mathrm{T}_{[m]}$ as above, any $1 \leq s \leq m$, and $k+m-1 \leq \frac{d+m-1}{2}$, and with $\mathrm{c}=\mathrm{c}(k, m, d)$ we have
(A) $\quad=\sum_{j=0}^{\lfloor m / 2\rfloor} \sum_{s=c-2 j+1}^{m-2 j} \sum_{\substack{S \subseteq[m] \\|S|=s}}\left(\begin{array}{c}m-s \\ 2 j\end{array}\right)\left(\widetilde{h}_{k+m-1-2 j}\left(\mathrm{~T}_{S}^{\circ}\right)-\frac{1}{2 j+1} \sum_{R \prec S} \widetilde{h}_{k+m-2-2 j}\left(\mathrm{~T}_{R}^{\circ}\right)\right)$
(B) $\quad+\sum_{j=0}^{\lfloor m / 2\rfloor} \sum_{\substack{S \subseteq \subseteq m] \\|S|=c-2 j}}\left(\begin{array}{c}m-|S|-1 \\ 2 j\end{array}\right)\left(\widetilde{h}_{k+m-1-2 j}\left(\mathrm{~T}_{S}^{\circ}\right)-\frac{m-S}{(m-|S|+1)(2 j+1)} \sum_{R \prec S} \widetilde{h}_{k+m-2-2 j}\left(\mathrm{~T}_{R}^{\circ}\right)\right)$.

Proof. We use induction on $m$, the case $m=1$ being trivial as the only term in the sums (A) and (B) is $\widetilde{h}_{k}\left(\mathrm{~T}_{[1]}^{\circ}\right)=h_{k}\left(\mathrm{~T}_{[1]}^{\circ}\right)+(-1)^{k}\left(\begin{array}{l}d \\ k\end{array}\right)=h_{k}\left(\mathrm{~T}_{[1]}\right)$. For $m>1$, note that by Proposition 5.13 and the definition of $\widetilde{g}$, we have

$$
h_{k+m-1}\left(\mathrm{~T}_{[m]}\right)=\sum_{S \subseteq[m]} \widetilde{g}_{k+m-1}^{\langle m-|S|\rangle}\left(\mathrm{T}_{S}^{\circ}\right)=\sum_{S \subseteq[m]} \sum_{i=0}^{m-|S|}(-1)^{i}\left(\begin{array}{c}
m-|S| \\
i
\end{array}\right) \widetilde{h}_{k+m-1-i}\left(\mathrm{~T}_{S}^{\circ}\right) .
$$

The coefficients of $\widetilde{h}_{*}\left(\mathrm{~T}_{S}^{\circ}\right)$ are summarized in Table 5.1. We split this last sum into two subsums, cf. Table 5.2, and obtain

$$
\begin{aligned}
& \sum_{S \subseteq[m]} \sum_{i=c-|S|+1}^{m-|S|}(-1)^{i}\left(\begin{array}{c}
m-|S| \\
i
\end{array}\right) \widetilde{h}_{k+m-1-i}\left(\mathrm{~T}_{S}^{\circ}\right) . \\
+ & \sum_{S \subseteq[m]} \sum_{i=0}^{c-|S|}(-1)^{i}\left(\begin{array}{c}
m-|S| \\
i
\end{array}\right) \widetilde{h}_{k+m-1-i}\left(\mathrm{~T}_{S}^{\circ}\right)
\end{aligned}
$$

\begin{tabular}{c|cccccc} 
& $|S|=m$ & $m-1$ & $m-2$ & $m-3$ & $m-4$ & $\cdots$ \\
\hline$k+m-1$ & 1 & 1 & 1 & 1 & 1 & $\cdots$ \\
$k+m-2$ & 0 & -1 & -2 & -3 & -4 & $\cdots$ \\
$k+m-3$ & 0 & 0 & 1 & 3 & 6 & $\cdots$ \\
$k+m-4$ & 0 & 0 & 0 & -1 & -4 & $\cdots$ \\
$\vdots$ & $\vdots$ & $\vdots$ & $\vdots$ & $\vdots$ & $\vdots$ & $\ddots$
\end{tabular}

Table 5.2. Example of the splitting into two subsums for $k+m-1=\frac{d+m-3}{2}$. Summands of $(\beta)$ highlighted.

Notice now that in the sum $(\beta)$, whenever $\widetilde{h}_{k^{\prime}}\left(\mathrm{T}_{S}^{\circ}\right)$ is evaluated, then $k^{\prime} \geq \frac{d+|S|-1}{2}$. Therefore, we may use the Dehn-Sommerville equations to substitute $(\beta)$ by

$$
\sum_{S \subseteq[m]} \sum_{i=0}^{\mathrm{c}-|S|}(-1)^{i}\left(\begin{array}{c}
m-|S| \\
i
\end{array}\right) h_{d+|S|-k-m}\left(\mathrm{~T}_{S}\right)
$$


with $d+|S|-k-m \leq \frac{d+|S|-1}{2}$. We may now evaluate $h_{|S|-m+i}\left(\mathrm{~T}_{S}\right)$ using the induction assumption, as illustrated in Table 5.3. We obtain, after also rewriting $\alpha$ to (A'), a decomposition

$$
\begin{aligned}
& h_{k+m-1}\left(\mathrm{~T}_{[m]}\right) \\
= & \sum_{S \subseteq[m]} \sum_{i=c-|S|+1}^{m-|S|}(-1)^{i}\left(\begin{array}{c}
m-|S| \\
i
\end{array}\right) \widetilde{h}_{k+m-1-i}\left(\mathrm{~T}_{S}^{\circ}\right) \\
+ & \sum_{j=0}^{m} \sum_{\substack{S \subseteq[m] \\
|S|=c-j}}^{m}(-1)^{j}\left(\begin{array}{c}
m-|S|-1 \\
j
\end{array}\right) \widetilde{h}_{k+m-1-j}\left(\mathrm{~T}_{S}^{\circ}\right) . \\
= & \sum_{j=0}^{m} \sum_{\substack{\mid \\
S=c-j+1}}^{m-j} \sum_{\substack{S \subseteq[m] \\
|S|=s}}(-1)^{j}\left(\begin{array}{c}
m-s \\
j
\end{array}\right) \widetilde{h}_{k+m-1-j}\left(\mathrm{~T}_{S}^{\circ}\right) \\
+ & \sum_{j=0}^{m} \sum_{\substack{S \subseteq[m] \\
|S|=\mathrm{c}-j}}(-1)^{j}\left(\begin{array}{c}
m-|S|-1 \\
j
\end{array}\right) \widetilde{h}_{k+m-1-j}\left(\mathrm{~T}_{S}^{\circ}\right) .
\end{aligned}
$$

\begin{tabular}{c|cccccc} 
& $|S|=m$ & $m-1$ & $m-2$ & $m-3$ & $m-4$ & $\cdots$ \\
\hline$k+m-1$ & 1 & 1 & 1 & 0 & 0 & $\cdots$ \\
$k+m-2$ & 0 & -1 & -2 & -2 & 0 & $\cdots$ \\
$k+m-3$ & 0 & 0 & 1 & 3 & 3 & $\cdots$ \\
$k+m-4$ & 0 & 0 & 0 & -1 & -4 & $\cdots$ \\
$\vdots$ & $\vdots$ & $\vdots$ & $\vdots$ & $\vdots$ & $\vdots$ & $\ddots$
\end{tabular}

Table 5.3. Applying the Dehn-Sommerville relations and induction simplifies the array.

We now pair summands with positive coefficient (specifically summands of $\widetilde{h}_{k+m-1-j}\left(\mathrm{~T}_{S}^{\circ}\right), j$ even) with summands $\widetilde{h}_{k+m-2-j}\left(\mathrm{~T}_{R}^{\circ}\right)$, where $R \prec S$ (see also Table 5.4 ).

\begin{tabular}{c|cccccc} 
& $|S|=m$ & $m-1$ & $m-2$ & $m-3$ & $m-4$ & $\cdots$ \\
\hline$k+m-1$ & 1 & 1 & 1 & 0 & 0 & $\cdots$ \\
$k+m-2$ & 0 & -1 & -2 & -2 & 0 & $\cdots$ \\
$k+m-3$ & 0 & 0 & 1 & 3 & 3 & $\cdots$ \\
$k+m-4$ & 0 & 0 & 0 & -1 & -4 & $\cdots$ \\
$\vdots$ & $\vdots$ & $\vdots$ & $\vdots$ & $\vdots$ & $\vdots$ & $\ddots$
\end{tabular}

Table 5.4. Pairing positive and negative terms of the sums (A') and (B'), corresponding to sets $S \subseteq[m]$ and $R \prec S$.

We see that the sum ( $\left.\mathrm{A}^{\prime}\right)$ is equal to

$$
\sum_{j=0}^{\lfloor m / 2\rfloor} \sum_{s=c-2 j+1}^{m-2 j} \sum_{\substack{S \subseteq[m] \\
|S|=s}}\left(\begin{array}{c}
m-s \\
2 j
\end{array}\right)\left(\widetilde{h}_{k+m-1-2 j}\left(\mathrm{~T}_{S}^{\circ}\right)-\frac{1}{2 j+1} \sum_{R \prec S} \widetilde{h}_{k+m-2-2 j}\left(\mathrm{~T}_{R}^{\circ}\right)\right)
$$

and the sum (B') equals

$$
\sum_{j=0}^{\lfloor m / 2\rfloor} \sum_{\substack{S \subseteq[m] \\
|S|=c-2 j}}\left(\begin{array}{c}
m-|S|-1 \\
2 j
\end{array}\right)\left(\widetilde{h}_{k+m-1-2 j}\left(\mathrm{~T}_{S}^{\circ}\right)-\frac{m-|S|}{(m-|S|+1)(2 j+1)} \sum_{R \prec S} \widetilde{h}_{k+m-2-2 j}\left(\mathrm{~T}_{R}^{\circ}\right)\right) .
$$


We conclude in particular that it is sufficient to know the various initial $h$-vector entries.

Corollary 5.15. The h-vector entries $h_{k+m-1}\left(\mathrm{~T}_{[m]}^{\circ}\right)$ for $k+m-1>\left\lfloor\frac{d+m-1}{2}\right\rfloor$ are determined by $\widetilde{h}_{k^{\prime}+m-1}\left(\mathrm{~T}_{S}^{\circ}\right)$ for $k^{\prime}+m-1 \leq\left\lfloor\frac{d+m-1}{2}\right\rfloor$ and $S \subseteq[m]$.

Passing from $h$-vectors to $f$-vectors and noting that Minkowski neighborly families maximize the 'small' $h$-entries in the sense of Corollary 5.11, we immediately conclude Proposition 5.3.

5.6. The Upper Bound Theorem for Minkowski sums. We can finally give sharp and explicit bounds for $h_{k}\left(\mathrm{~T}_{[m]}^{\circ}\right)$. Let us define the functions

$$
\widetilde{\omega}^{\langle\ell\rangle}: \mathbb{Z}^{[m]} \times \mathbb{Z} \times \mathbb{Z} \longrightarrow \mathbb{Z}, m \geq 0, \ell \geq 0
$$

(where we abbreviate $\widetilde{\omega}:=\widetilde{\omega}^{\langle 0\rangle}$ ), and

$$
\omega: \mathbb{Z}^{[m]} \times \mathbb{Z} \times \mathbb{Z} \longrightarrow \mathbb{Z}, m \geq 0
$$

by the following conditions:

(a) Basic relation: For all $k, d \geq 0$

$$
\widetilde{\omega}^{\langle\ell\rangle}(\cdot, \cdot, k)=\widetilde{\omega}^{\langle\ell-1\rangle}(\cdot, \cdot, k)-\widetilde{\omega}^{\langle\ell-1\rangle}(\cdot, \cdot, k-1) ;
$$

(b) Linearity: For all $-m+1 \leq k \leq d$

$$
\omega(\alpha, d, k+m-1)=\sum_{S \subseteq[m]} \widetilde{\omega}^{\langle m-|S|\rangle}\left(\alpha_{S}, d, k+m-1\right),
$$

where $\alpha_{S} \in \mathbb{Z}^{S}$ is the restriction of $\alpha \in \mathbb{Z}^{[m]}$ to the index set $S \subseteq[m]$;

(c) Dehn-Sommerville relation: For all $-m+1 \leq k \leq d$

$$
\omega(\alpha, d, d-k)=\widetilde{\omega}(\alpha, d, k+m-1) ;
$$

(d) Initial terms: For $k+m-1 \leq \frac{d+m-1}{2}$,

$$
\widetilde{\omega}(\alpha, d, k+m-1)=\sum_{\varnothing \neq S \subseteq[m]}(-1)^{m-|S|}\left(\begin{array}{c}
\left|\alpha_{S}\right|-d+k-1 \\
k+m-1
\end{array}\right) .
$$

Using the fact that $\omega$ and $\widetilde{\omega}$ encode the $h$-vector for Minkowski neighborly families, whose $h$-vectors satisfy relations (a)-(d) and which exist by Theorem 5.2, we conclude consistency of these relations. By linearity (a) and the definition of the initial terms $(\mathrm{d})$, we see that:

Lemma 5.16. For all $k+m \leq \frac{d+m-1}{2}$

$$
\begin{aligned}
& (k+m) \widetilde{\omega}(\alpha, d, k+m) \\
= & \left(f_{0}\left(\mathrm{~T}_{[m]}\right)-d+k\right) \widetilde{\omega}(\alpha, d, k+m)\left(\mathrm{T}_{[m]}^{\circ}\right) \\
+ & \sum_{i \in[m]} f_{0}\left(\mathrm{~T}_{\{i\}}\right) \widetilde{\omega}^{\langle 1\rangle}(\alpha, d, k+m-1) .
\end{aligned}
$$

The next lemma holds all the missing ingredients necessary for resolving the Upper Bound Conjecture for Minkowski sums.

Lemma 5.17. Let $P_{[m]}=\left(P_{1}, \ldots, P_{m}\right)$ be a family of simplicial d-polytopes in relatively general position in $\mathbb{R}^{d}$ with Cayley complex $\mathrm{T}_{[m]}=\mathrm{T}\left(P_{1}, \ldots, P_{m}\right)$. Let $-m+1 \leq k \leq d$ such that $k+m-1 \leq\left\lfloor\frac{d+m-1}{2}\right\rfloor$ and let $0 \leq \delta \leq \frac{d+1}{d-1}$ be any real parameter. Then

$$
\begin{aligned}
& \widetilde{g}_{k+m-1}\left(\mathrm{~T}_{[m]}^{\circ}\right)-\delta \sum_{S \prec[m]} \widetilde{g}_{k+m-2}\left(\mathrm{~T}_{S}^{\circ}\right) \\
\leq & \widetilde{\omega}^{\langle 1\rangle}\left(f_{0}\left(P_{[m]}\right), d, k+m-1\right)-\delta \sum_{S \prec[m]} \widetilde{\omega}^{\langle 1\rangle}\left(f_{0}\left(P_{S}\right), d, k+m-2\right)
\end{aligned}
$$

If $\delta<\frac{d+1}{d-1}$, then equality holds if and only if it holds for each summand separately. 
Remark 5.18. Alternatively, and as an application of Theorem 4.24, one can prove a result that can be used just as well to prove the Upper Bound Theorem on Minkowski sums: For a family $P_{[m]}$ in $\mathbb{R}^{d}$ of polytopes in relative general position, and a monotone increasing family of nonnegative real parameters $\left(\delta_{s}\right), 0 \leq s \leq m$, we have

$$
\sum_{S \subseteq[m]}(-1)^{m-\# S} \delta_{|S|} g_{k+|S|-1}\left(\mathrm{~T}_{S}^{\circ}\right) \leq \sum_{S \subseteq[m]}(-1)^{m-\# S} \delta_{|S|} \widetilde{\omega}^{\langle 1\rangle}\left(f_{0}\left(P_{S}\right), d, k+|S|-1\right),
$$

with equality if and only if it holds for each summand. For the proof, notice that following Theorem 4.24, we have for $v \in P_{i}$ an inequality

$$
\sum_{i \in S \subseteq[m]}(-1)^{m-\# S} h_{k+|S|-1}\left(\operatorname{lk}\left(v, \mathrm{~T}_{S}^{\circ}\right)\right) \leq \sum_{i \in S \subseteq[m]}(-1)^{m-\# S}\left(h_{k+|S|-1}\left(\mathrm{~T}_{S}^{\circ}\right)-g_{k+|S|-1}\left(\mathrm{~T}_{S \backslash\{i\}}^{\circ}\right)\right),
$$

sum over all $v$ in $\mathrm{T}_{[m]}$ and argue as in the proof of Lemma 5.17 below.

Proof of Lemma 5.17. Using Theorem 5.10, we obtain

$$
\begin{aligned}
& \widetilde{g}_{k+m-1}\left(\mathrm{~T}_{[m]}^{\circ}\right)-\delta \sum_{S \prec[m]} \widetilde{g}_{k+m-2}\left(\mathrm{~T}_{S}^{\circ}\right) \\
\leq & \left(\frac{f_{0}\left(\mathrm{~T}_{[m]}\right)-d-m}{k+m-1}\right) \widetilde{g}_{k+m-2}\left(\mathrm{~T}_{[m]}^{\circ}\right)+\sum_{S \prec[m]}\left(\frac{f_{0}\left(\mathrm{~T}_{[m] \backslash S}\right)}{k+m-1}-\delta\right) \widetilde{g}_{k+m-2}\left(\mathrm{~T}_{S}^{\circ}\right)
\end{aligned}
$$

As the equality is only nontrivial if $k \geq 1$ and therefore

$$
m \leq \frac{d+m-1}{2} \Longleftrightarrow m \leq d-1,
$$

we may assume that $k+m-1 \leq d-1$. Hence, as $f_{0}\left(P_{i}\right) \geq d+1$ and $k \leq d$, we have $\frac{f_{0}\left(\mathrm{~T}_{[m] \backslash S}\right)}{k+m-1}-\delta \geq 0$, and the latter sum is bounded from above by $\omega$ :

$$
\begin{aligned}
& \left(\frac{f_{0}\left(\mathrm{~T}_{[m]}\right)-d-m}{k+m-1}\right) \widetilde{\omega}^{\langle 1\rangle}\left(f_{0}\left(P_{[m]}\right), d, k+m-2\right) \\
+ & \sum_{S \prec[m]}\left(\frac{f_{0}\left(\mathrm{~T}_{[m] \backslash S}\right)}{k+m-1}-\delta\right) \widetilde{\omega}^{\langle 1\rangle}\left(f_{0}\left(P_{S}\right), d, k+m-2\right) \\
= & \widetilde{\omega}^{\langle 1\rangle}\left(f_{0}\left(P_{[m]}\right), d, k+m-1\right)-\delta \sum_{S \prec[m]} \widetilde{\omega}^{\langle 1\rangle}\left(f_{0}\left(P_{S}\right), d, k+m-2\right)
\end{aligned}
$$

where the last equality follows from Lemma 5.16. The equality case follows directly from Theorem 5.10.

We summarize the upper bounds in the following theorem. We let c as in Lemma 5.14.

Theorem 5.19. Let $P_{[m]}=\left(P_{1}, \ldots, P_{m}\right)$ be a family of simplicial d-polytopes in relatively general position in $\mathbb{R}^{d}$ with $\mathbf{n}=f_{0}\left(P_{[m]}\right)$. For the corresponding Cayley complex $\mathrm{T}_{[m]}=\mathrm{T}\left(P_{[m]}\right)$ the following holds

(1) $\omega$ is an upper bound:

(a) for any $-m+1 \leq k \leq d$ with $k+m-1 \leq \frac{d+m-1}{2}$

$$
\widetilde{h}_{k+m-1}\left(\mathrm{~T}_{[m]}^{\circ}\right) \leq \widetilde{\omega}(\mathbf{n}, d, k+m-1),
$$

(b) for any $-m+1 \leq k \leq d$ with $k+m-1 \leq \frac{d+m-1}{2}$, we have

$$
h_{k+m-1}\left(\mathrm{~T}_{[m]}\right) \leq \omega(\mathbf{n}, d, k+m-1),
$$

(2) Equality cases:

(a) equality holds up to some $k_{0}+m-1$ in (1a) if and only if, for all $S \subseteq[m]$, all non-faces of $\mathrm{T}_{[m]}$ of cardinality $k_{0}+m-1$ are supported in some $\mathrm{V}\left(\mathrm{T}_{R}\right), R \subsetneq S$.

(b) equality holds up to some $k_{0}+m-1$ in (1b) if and only if, for a nonface of cardinality $k_{0}+m-1-q$ in $\mathrm{T}_{S}, \# S \geq \mathrm{c}\left(k_{0}, m, d\right)-q$ is a nonface are supported in some $\mathrm{V}\left(\mathrm{T}_{R}\right), R \subsetneq S$. 
(3) Tightness: there is a pure collection of $m$ polytopes $Q_{i}$ in $\mathbb{R}^{d}$ with $f_{0}\left(Q_{[m]}\right)=f_{0}\left(P_{[m]}\right)$ such that for all $-m+1 \leq k \leq d$ with $k+m-1 \leq \frac{d+m-1}{2}$

$$
\begin{aligned}
& \widetilde{h}_{k+m-1}\left(\mathrm{~T}_{[m]}^{\circ}\left(Q_{i}\right)\right)=\widetilde{\omega}\left(f_{0}\left(Q_{[m]}\right), d, k+m-1\right), \text { and } \\
& h_{k+m-1}\left(\mathrm{~T}_{[m]}\left(Q_{i}\right)\right)=\omega\left(f_{0}\left(Q_{[m]}\right), d, k+m-1\right) .
\end{aligned}
$$

Remark 5.20. Note that to bound $\widetilde{h}_{k+m-1}\left(\mathrm{~T}_{[m]}^{\circ}\right)$ for $k+m-1 \geq \frac{d+m-1}{2}$ we use the Dehn-Sommerville inequalities, and can resolve to bounding

$$
h_{d-k}\left(\mathrm{~T}_{[m]}\right) \text {. }
$$

For instance, if we want to bound the number of vertices in a Minkowski sum, then we are really asked to bound $\widetilde{h}_{m}\left(\mathrm{~T}_{[m]}^{\circ}\right)$ or equivalently $h_{d-1}\left(\mathrm{~T}_{[m]}\right)$. The tightness condition of $(2 \mathrm{~b})$ says that this is attained if and only if any subface of cardinality $d-1-q$ in $\mathrm{T}_{S}, \# S \geq d-1-q$ is contained in a subsummand.

Proof. Notice first that claims (1a) and (2a) are verbatim special cases of Theorem 5.11. Therefore, the proof of the stated claims splits into two parts: We first prove (1b) and (2b), and then we address the question of tightness.

Claims (1b) and (2b): By Lemma 5.14, we have

$$
\begin{aligned}
& h_{k+m-1}\left(\mathrm{~T}_{[m]}\right) \\
& =\sum_{j=0}^{\left\lfloor\frac{m}{2}\right\rfloor} \sum_{s=c-2 j+1}^{m-2 j} \sum_{\substack{S \subseteq[m] \\
|S|=s}}\left(\begin{array}{c}
m-s \\
2 j
\end{array}\right)\left(\widetilde{h}_{k+m-1-2 j}\left(\mathrm{~T}_{S}^{\circ}\right)-\frac{1}{2 j+1} \sum_{R \prec S} \widetilde{h}_{k+m-2-2 j}\left(\mathrm{~T}_{R}^{\circ}\right)\right) \\
& +\sum_{j=0}^{\left\lfloor\frac{m}{2}\right\rfloor} \sum_{\substack{S \subseteq[m] \\
|S|=c-2 j}}\left(\begin{array}{c}
m-|S|-1 \\
2 j
\end{array}\right)\left(\widetilde{h}_{k+m-1-2 j}\left(\mathrm{~T}_{S}^{\circ}\right)-\frac{m-|S|}{(m-|S|+1)(2 j+1)} \sum_{R \prec S} \widetilde{h}_{k+m-2-2 j}\left(\mathrm{~T}_{R}^{\circ}\right)\right) .
\end{aligned}
$$

Now, clearly, $\frac{1}{2 j+1} \leq 1<\frac{d+1}{d-1}$, so that we can estimate the sums (A) and (B) using Lemma 5.17, obtaining

$$
\begin{aligned}
& \widetilde{h}_{k+m-1}\left(\mathrm{~T}_{[m]}\right) \\
\leq & \sum_{j=0}^{\left\lfloor\frac{m}{2}\right\rfloor} \sum_{s=c-2 j+1}^{m-2 j} \sum_{\substack{S \subseteq[m] \\
|S|=s}}\left(\begin{array}{c}
m-s \\
2 j
\end{array}\right)\left(\widetilde{\omega}\left(f_{0}\left(P_{S}\right), d, k_{j}^{\prime}\right)-\frac{1}{2 j+1} \sum_{R \prec S} \widetilde{\omega}\left(f_{0}\left(P_{[m]}\right)_{R}, d, k_{j}^{\prime}-1\right)\right) \\
+ & \sum_{j=0}^{\left\lfloor\frac{m}{2}\right\rfloor} \sum_{\substack{S \subseteq[m] \\
|S|=c-2 j}}\left(\begin{array}{c}
m-|S|-1 \\
2 j
\end{array}\right)\left(\widetilde{\omega}\left(f_{0}\left(P_{S}\right), d, k_{j}^{\prime}\right)-\frac{m-|S|}{(m-|S|+1)(2 j+1)} \sum_{R \prec S} \widetilde{\omega}\left(f_{0}\left(P_{[m]}\right)_{R}, d, k_{j}^{\prime}-1\right)\right)
\end{aligned}
$$

where we abbreviate $k_{j}^{\prime}:=k+m-1-2 j$. Since $\widetilde{\omega}$ satisfies linearity and the Dehn-Sommerville symmetries as well, we can reverse the logic of Lemma 5.14, the latter sums equals $\omega\left(f_{0}\left(P_{[m]}\right), d, k+m-1\right)$ of $(1 \mathrm{~b})$. Equality only holds if it holds in the application of Lemma 5.17, therefore also concluding the proof of claim $(2 \mathrm{~b})$.

Claim (3): By Theorem 5.19(2a) and (2b), it suffices to show that there is a Minkowski neighborly family $Q_{[m]}$ of simplicial $d$-polytopes in $\mathbb{R}^{d}$ with $f_{0}\left(Q_{[m]}\right)=\mathbf{n}$. Such a family is provided by Theorem $5.2(\mathrm{ii})$.

\section{Minkowski sums of nOnpure COLlections}

In Section 5 , a basic assumption on the collection $P_{[m]}=\left(P_{1}, \ldots, P_{m}\right)$ of polytopes in $\mathbb{R}^{d}$ was that $f_{0}\left(P_{i}\right) \geq d+1$ for all $i=1, \ldots, m$. Minkowski sums of nonpure collections, i.e., collections $P_{[m]}$ such that $f_{0}\left(P_{i}\right)<d+1$ for some $i$, are however of importance. The simplest case is when all summands have exactly two vertices. In this case the resulting Minkowski sum is a zonotope and the corresponding Upper Bound Theorem is well-known [Buc43] (and in essence goes back to Steiner [Ste26]). In this section we will give an extension of the Upper Bound Theorem for Minkowski sums to nonpure collections. This is a nontrivial step and the reader will observe the increase in complexity of the arguments and especially notation. For this reason we 
devote a separate section for the nonpure situation. Nevertheless, the basic line of reasoning remains the same and we only sketch the main amendments.

Let us notice that if $\left|P_{[m]}\right|$ maximize the number of $k$-faces, then the polytopes $P_{i}$ are simplicial and in relatively general position. In particular, if $P_{i}$ has fewer than $d+1$ vertices then genericity implies that $P_{i}$ is a $\left(f_{0}\left(P_{i}\right)-1\right)$-simplex. For the nonpure UBPM, we need to introduce an additional parameter: For a family $P_{[m]}$ of $m$ polytopes in $\mathbb{R}^{d}$ with $\mathbf{n}=f_{0}\left(P_{[m]}\right)$, let us abbreviate the dimension of the Minkowski sum of the subfamily $P_{S}$ by $\xi\left(P_{S}\right)$. Note that this quantity is determined purely in terms of the vector $\mathbf{n}$ of vertex numbers:

$$
\xi\left(P_{S}\right)=\xi\left(\mathbf{n}_{S}\right):=\min \left(d,\left|\mathbf{n}_{S}\right|-|S|\right) .
$$

We start with an analogue of Theorem 5.2 that applies to nonpure collections.

Theorem 6.1. Let $m, d \geq 1$ be fixed.

(i) There is no Minkowski $(k, \ell)$-neighborly family $P_{[m]}$ in $\mathbb{R}^{d}$ for $k+\ell-1>\left\lfloor\frac{\xi\left(P_{[m]}\right)+\ell-1}{2}\right\rfloor$.

(ii) For all $\mathbf{n} \in \mathbb{Z}_{\geq 0}^{m}$ there is a family $P_{[m]}$ in $\mathbb{R}^{d}$ with $f_{0}\left(N_{[m]}\right)=\mathbf{n}$ that is Minkowski $(k, \ell)$-neighborly for all $\ell \leq m$ and $\ell-1 \leq k+\ell-1 \leq\left\lfloor\frac{\xi\left(P_{[m]}\right)+\ell-1}{2}\right\rfloor$.

The first claim follows from an analogues statement to Proposition 5.6. The second statement follows again from the work of Matschke-Pfeifle-Pilaud [MPP11]. We continue to call the collections of Theorem 6.1(ii) Minkowski neighborly. Similar to the case of the UBT for spheres, we can use Minkowski neighborly polytopes to abbreviate the UBTM, because their $f$-vectors depend on $m, d$ and $f_{0}\left(P_{[m]}\right)$ only.

Proposition 6.2. If $P_{[m]}, P_{[m]}^{\prime}$ are two Minkowski neighborly families of m simplicial polytopes with $f_{0}\left(P_{[m]}\right)=$ $f_{0}\left(P_{[m]}^{\prime}\right)$, then $f_{k}\left(\left|P_{[m]}\right|\right)=f_{k}\left(\left|P_{[m]}^{\prime}\right|\right)$ for all $0 \leq k \leq d$.

At this point, let us remark two curious properties that make our life simpler.

Observation 6.3. Let $P_{[m]}$ be nonpure collection of relative general position polytopes in $\mathbb{R}^{d}$.

(1) If the Minkowski sum of polytopes in relatively general position is not full-dimensional, then $\left|P_{[m]}\right| \cong$ $P_{1} \times P_{2} \times \cdots \times P_{m}$. In this case we say that $P_{[m]}$ is deficient.

(2) If $\operatorname{dim} P_{i}=0$ for some $i \in[m]$, then $\left|P_{[m]}\right|$ is a translate of $\left|P_{[m] \backslash i}\right|$.

We recover Buck's Theorem on zonotopes [Buc43].

Corollary 6.4. Any family $P_{[m]}$ of $m$ segments in relatively general position in $\mathbb{R}^{d}$ is Minkowski neighborly. In particular, the $f$-vector of the zonotope $\left|P_{[m]}\right|$ only depend on $m$ and $d$.

Proof. Use Proposition 6.2 and the fact that all families of at most $d$ edges in $\mathbb{R}^{d}$ is deficient.

For the UBT for Minkowski sums, we define for $m, d \geq 1$ and $\mathbf{n} \in \mathbb{Z}_{\geq 0}^{m}$

$$
\operatorname{nb}_{i}(d, m, \mathbf{n}):=f_{i}\left(\left|P_{[m]}\right|\right)
$$

where $P_{[m]}$ is any Minkowski neighborly family of $m$ simplicial polytopes in $\mathbb{R}^{d}$ with $\mathbf{n}=f_{0}\left(P_{[m]}\right)$.

Theorem 6.5 (Upper Bound Theorem for Minkowski sums of general families). Let $m, d \geq 1$ and $\mathbf{n} \in \mathbb{Z}_{\geq 0}^{m}$. If $P_{[m]}=\left(P_{1}, \ldots, P_{m}\right)$ is a family of $m$ polytopes in $\mathbb{R}^{d}$ with $P_{[m]}=\mathbf{n}$, then

$$
f_{k}\left(\left|P_{[m]}\right|\right) \leq \operatorname{nb}_{k}(d, m, \mathbf{n})
$$

for all $k=0, \ldots, d-1$. Moreover, the family $P_{[m]}$ is Minkowski neighborly if and only if equality holds for the number of facets.

The remainder of this section will provide the proof of Theorem 6.5. We only sketch the line of reasoning for the main points. 
6.1. Initial terms of the $h$-vector. We start with a replacement for Theorem 5.10 that applies to nonpure collections.

Theorem 6.6. Let $P_{[m]}=\left(P_{1}, \ldots, P_{m}\right)$ be a family of simplicial polytopes in relatively general position in $\mathbb{R}^{d}$. Let $\mathrm{T}_{[m]}$ be the corresponding Cayley complex and $e=\xi\left(P_{[m]}\right)$. Then, for every $k$,

(a) and for every $i \in[m]$ and $v$ vertex of $P_{i}$

$$
h_{k}\left(\operatorname{lk}\left(v, \mathrm{~T}_{[m]}^{\circ}\right)\right) \leq h_{k}\left(\mathrm{~T}_{[m]}^{\circ}\right)+h_{k}^{\langle e+m-1\rangle}\left(\mathrm{T}_{[m] \backslash\{i\}}^{\circ}\right), \text { and }
$$

(b)

$$
(k+m) g_{k+m}\left(\mathrm{~T}_{[m]}^{\circ}\right) \leq\left(f_{0}\left(\mathrm{~T}_{[m]}\right)-e-m\right) h_{k+m-1}\left(\mathrm{~T}_{[m]}^{\circ}\right)+\sum_{i \in[m]} f_{0}\left(\mathrm{~T}_{\{i\}}\right) h_{k+m-1}^{\langle e+m-1\rangle}\left(\mathrm{T}_{[m] \backslash\{i\}}^{\circ}\right) .
$$

(c) We have

$$
(i+|S|) h_{i+|S|}\left(\mathrm{T}_{S}^{\circ}\right)=\left(f_{0}\left(\mathrm{~T}_{S}\right)-d+i\right) h_{i+|S|-1}\left(\mathrm{~T}_{S}^{\circ}\right)+\sum_{i \in S} f_{0}\left(\mathrm{~T}_{\{i\}}\right) h_{i+|S|-1}^{\left\langle\xi\left(P_{S}\right)\right\rangle}\left(\mathrm{T}_{S \backslash\{i\}}^{\circ}\right)
$$

for all $i \leq k_{0}$ and $S \subseteq[m]$ if and only if all non-faces of $\mathrm{T}_{S}$ of dimension $<k_{0}+|S|$ are supported in some $\mathrm{V}\left(\mathrm{T}_{R}\right), R \subsetneq S$.

Proof. It suffices to prove (a) and characterize equality in this case; the other inequalities are obtained by simply summing over all vertices. To prove the inequality, notice that by Lemma $1.2, h_{k+m-1}\left(\mathrm{k}\left(v, \mathrm{~T}_{[m]}^{\circ}\right)\right)=$ $h_{k+m-1}\left(\operatorname{st}\left(v, \mathrm{~T}_{[m]}^{\circ}\right)\right)$ and

$$
h_{k+m-1}\left(\mathrm{~T}_{[m]}^{\circ}\right)+h_{k+m-1}^{\langle e+m-1\rangle}\left(\mathrm{T}_{[m] \backslash\{i\}}^{\circ}\right)=h_{k+m-1}\left(\mathrm{~T}_{[m]}^{\circ}(i)\right)
$$

where we define

$$
\mathrm{T}_{[m]}^{\circ}(i):=\left(\mathrm{T}_{[m]}, \bigcup_{i \in S \subsetneq[m]} \mathrm{T}_{S}\right) .
$$

Hence it suffices to prove that $h_{k+m-1}\left(\operatorname{st}\left(v, \mathrm{~T}_{[m]}^{\circ}\right)\right) \leq h_{k+m-1}\left(\mathrm{~T}_{[m]}^{\circ}(i)\right)$. For this, let $\mathrm{C}=\operatorname{Cay}\left(P_{1}, \ldots, P_{m}\right)$, together with the faces

$$
\mathrm{C}_{S}:=\operatorname{conv}\left(\bigcup_{i \in S} P_{i}+e_{i}\right) \text { for } S \subsetneq[m] .
$$

The complex $C(i)=\bigcup_{i \in S \subsetneq[m]} \mathrm{C}_{S}$ is a PL ball and st $(v, \partial \mathrm{C})$ is a PL ball of the same dimension contained in it, so that $(\partial \mathrm{C}, \operatorname{st}(v, \partial \mathrm{C}))$ is Cohen-Macaulay by Theorem 1.9. Hence

$$
(C(i), \operatorname{st}(v, \partial \mathrm{C})) \cong\left(C(i), \operatorname{st}\left(v, \mathrm{~T}_{[m]}\right) \cup \bigcup_{i \in S \subsetneq[m]} \mathrm{T}_{S}\right) \cong\left(\mathrm{T}_{[m]}^{\circ}(i), \operatorname{st}\left(v, \mathrm{~T}_{[m]}^{\circ}\right)\right)
$$

where the last complex is the complement of $\operatorname{st}\left(v, \mathrm{~T}_{[m]}^{\circ}\right)$ in $\mathrm{T}_{[m]}^{\circ}(i)$. Hence

$$
h_{k+m-1}\left(\operatorname{st}\left(v, \mathrm{~T}_{[m]}^{\circ}\right)\right)+h_{k+m-1}\left(\mathrm{~T}_{[m]}^{\circ}(i), \operatorname{st}\left(v, \mathrm{~T}_{[m]}^{\circ}\right)=h_{k+m-1}\left(\mathrm{~T}_{[m]}^{\circ}(i)\right)\right.
$$

by linearity of the $h$-vector and $h_{k+m-1}\left(\mathrm{~T}_{[m]}^{\circ}(i), \operatorname{st}\left(v, \mathrm{~T}_{[m]}^{\circ}\right) \geq 0\right.$ by Cohen-Macaulayness. Equality, i.e. $h_{k+m-1}\left(\mathrm{~T}_{[m]}^{\circ}(i), \operatorname{st}\left(v, \mathrm{~T}_{[m]}^{\circ}\right)=0\right.$, holds up to some $k_{0}+m-1$ if $\mathrm{M}\left[\mathrm{T}_{[m]}^{\circ}(i), \operatorname{st}\left(v, \mathrm{~T}_{[m]}^{\circ}\right)\right]$ is generated in degree $>k_{0}+m-1$. We conclude by iteratively applying the same argument to all subsets $S \subseteq[m]$.

We conclude as in Section 5.4:

Corollary 6.7. Let $P_{[m]}=\left(P_{1}, \ldots, P_{m}\right)$ be a family of $m$ simplicial in relatively general position and let $\mathrm{T}_{[m]}^{\circ}$ be the corresponding relative Cayley complex, and let $e=\xi\left(P_{[m]}\right)$. Then for all $-m+1 \leq k \leq d$

$$
h_{k+m-1}\left(\mathrm{~T}_{[m]}^{\circ}\right) \leq \sum_{S \subseteq[m]}(-1)^{m-|S|}\left(\begin{array}{c}
f_{0}\left(\mathrm{~T}_{S}\right)-e+k-1 \\
k+m-1
\end{array}\right) .
$$

Equality holds for some $k_{0}+m-1$ if and only if all non-faces of $\mathrm{T}_{[m]}$ of dimension $<k_{0}+m-1$ are supported in some $\mathrm{V}\left(\mathrm{T}_{S}\right), S \subsetneq[m]$. 
6.2. The Dehn-Sommerville formula and other linear relations. The most challenging part of the Upper Bound Problem for Minkowski sums are the Dehn-Sommerville relations. Recall that we can assume that $\operatorname{dim}\left|P_{[m]}\right|=d$. The following is a simple corollary of Proposition 1.4.

Lemma 6.8. Let $\mathrm{T}=\mathrm{T}\left(P_{[m]}\right)$ be the Cayley complex for $P_{[m]}$. Then, for all $-m+1 \leq k \leq d$

$$
h_{d-k}\left(\mathrm{~T}_{[m]}\right)=\widetilde{h}_{k+m-1}\left(\mathrm{~T}_{[m]}^{\circ}\right)+\sum_{\substack{S \subseteq[m] \\
\xi\left(\bar{P}_{S}\right)<d}}(-1)^{k}\left(\begin{array}{c}
d+m-1 \\
m+k+\xi\left(P_{S}\right)
\end{array}\right) .
$$

The important point to note here is that the correction term $\sum_{\substack{S \subseteq[m] \\ \xi\left(P_{S}\right)<d}}(-1)^{k}\left(\begin{array}{c}d+m-1 \\ m+k+\xi\left(P_{S}\right)\end{array}\right)$ only depends on $k, d, m$ and $f_{0}\left(P_{[m]}\right)$ but not on the combinatorial type of the Cayley polytope. Now, we note that by linearity of the $g$-vector:

Proposition 6.9. For $\mathrm{T}_{[m]}$ as above, any $\ell \geq 0$, and any $1 \leq s \leq m$, we have

$$
h_{k+m-1}\left(\mathrm{~T}_{[m]}\right)=\sum_{S \subseteq[m]} \widetilde{g}_{k+m-1}^{\left\langle m-|S|+\xi\left(P_{[m]}\right)-\xi\left(P_{S}\right)\right\rangle}\left(\mathrm{T}_{S}^{\circ}\right) .
$$

Recall that by Observation 6.3(i), the combinatorial type of $\mathrm{T}_{S}^{\circ}$ is determined by $f_{0}\left(P_{S}\right)$ (and $d$ ) if $\xi\left(P_{S}\right)<d$. Note furthermore that if $\xi\left(P_{S}\right)<d$, then $\xi\left(P_{R}\right)<d$ for all $R \subseteq S$. We obtain, with arguments analogous to Lemma 5.14.

Lemma 6.10. For $\mathrm{T}_{[m]}, \xi\left(P_{[m]}\right)=d$ as above, any $1 \leq s \leq m$, any $k+m-1 \leq \frac{d+m-1}{2}$, and with $\mathrm{c}=\mathrm{c}(k, m, d)$ we have

$$
\begin{aligned}
& \sum_{j=0} \sum_{s=c-2 j+1} \sum_{\substack{S \subseteq[m] \\
|S|=s \\
\xi(P S)=d}}\left(\begin{array}{c}
m-s\rfloor \\
2 j
\end{array}\right)\left(\widetilde{h}_{k+m-1-2 j}\left(\mathrm{~T}_{S}^{\circ}\right)-\frac{1}{2 j+1} \sum_{\substack{R \prec S \\
\xi\left(P_{R}\right)=d}} \widetilde{h}_{k+m-2-2 j}\left(\mathrm{~T}_{R}^{\circ}\right)\right) \\
+ & \sum_{j=0}^{\lfloor-2 j} \sum_{\substack{S \subseteq[m] \\
|S|=c-2 j \\
\xi\left(P_{S}\right)=d}}^{\lfloor m / 2\rfloor}\left(\begin{array}{c}
m-|S|-1 \\
2 j
\end{array}\right)\left(\widetilde{h}_{k+m-1-2 j}\left(\mathrm{~T}_{S}^{\circ}\right)-\frac{m-|S|}{(m-|S|+1)(2 j+1)} \sum_{\substack{R \prec S \\
\xi\left(P_{R}\right)=d}} \widetilde{h}_{k+m-2-2 j}\left(\mathrm{~T}_{R}^{\circ}\right)\right) \\
+ & \gamma\left(f_{0}\left(P_{[m]}\right), m, d, k\right) .
\end{aligned}
$$

where the correction term $\gamma$ only depends on $f_{0}\left(P_{[m]}\right), m, d$ and $k$, but not on the combinatorial type of $\operatorname{Cay}\left(P_{1}, \cdots, P_{m}\right)$.

6.3. The Upper Bound Theorem for nonpure Minkowski sums. We finally conclude the Upper Bound Theorem for pure Minkowski sums: We define

$$
\widetilde{\nu}^{\langle\ell\rangle}: \mathbb{Z}^{[m]} \times \mathbb{Z} \times \mathbb{Z} \longrightarrow \mathbb{Z}, m \geq 0, \ell \geq 0
$$

and

$$
\nu: \mathbb{Z}^{[m]} \times \mathbb{Z} \times \mathbb{Z} \longrightarrow \mathbb{Z}, m \geq 0
$$

by the following conditions:

(a) Basic relation: For all $k, \ell \geq 0$

$$
\widetilde{\nu}^{\langle\ell\rangle}(\cdot, \cdot, k)=\widetilde{\nu}^{\langle\ell-1\rangle}(\cdot, \cdot, k)-\widetilde{\nu}^{\langle\ell-1\rangle}(\cdot, \cdot, k-1) ;
$$

(b) Linearity: For all $-m+1 \leq k \leq d$

$$
\nu(\alpha, d, k+m-1)=\sum_{S \subseteq[m]} \widetilde{\nu}^{\left\langle m-|S|+\xi(\alpha)-\xi\left(\alpha_{S}\right)\right\rangle}\left(\alpha_{S}, d, k+m-1\right) .
$$


(c) Dehn-Sommerville relation: For all $-m+1 \leq k \leq d$

$$
\nu(\alpha, d, d-k)=\widetilde{\nu}(\alpha, d, k+m-1)+\sum_{\substack{S \subseteq[m] \\
\xi\left(\alpha_{S}\right)<d}}(-1)^{k}\left(\begin{array}{c}
d+m-1 \\
m+k+\xi\left(\alpha_{S}\right)
\end{array}\right) ;
$$

(d) Initial terms: For $k+m-1 \leq \frac{\xi\left(\alpha_{[m]}\right)+m-1}{2}$, we have

$$
\widetilde{\nu}(\alpha, d, k+m-1)=\sum_{\varnothing \neq S \subseteq[m]}(-1)^{m-|S|}\left(\begin{array}{c}
\left|\alpha_{S}\right|-\xi\left(\alpha_{S}\right)+k-1 \\
k+m-1
\end{array}\right) .
$$

With this we obtain the desired UBT for Minkowski sums of nonpure collections.

Theorem 6.11. Let $P_{[m]}=\left(P_{1}, \ldots, P_{m}\right)$ be a family of simplicial polytopes in relatively general position in $\mathbb{R}^{d}$ with $\mathbf{n}=f_{0}\left(P_{[m]}\right)$ and $\operatorname{dim}\left|P_{[m]}\right|=d$. For the corresponding Cayley complex $\mathrm{T}_{[m]}=\mathrm{T}\left(P_{[m]}\right)$ the following holds

(1) $\nu$ is an upper bound:

(a) for any $-m+1 \leq k \leq d$ with $k+m-1 \leq \frac{d+m-1}{2}$

$$
\widetilde{h}_{k+m-1}\left(\mathrm{~T}_{[m]}^{\circ}\right) \leq \widetilde{\nu}(\mathbf{n}, d, k+m-1),
$$

(b) for any $-m+1 \leq k \leq d$ with $k+m-1 \leq \frac{d+m-1}{2}$

$$
h_{k+m-1}\left(\mathrm{~T}_{[m]}\right) \leq \nu(\mathbf{n}, d, k+m-1),
$$

(2) Equality cases:

(a) equality holds up to some $k_{0}+m-1$ in (1a) if and only if, for all $S \subseteq[m]$, all non-faces of $\mathrm{T}_{S}$ of cardinality $\leq k_{0}+|S|-1$ are supported in some $\mathrm{V}\left(\mathrm{T}_{R}\right), R \subsetneq S$.

(b) equality holds up to some $k_{0}+m-1$ in (1b) if and only if, for a nonface of cardinality $k_{0}+m-1-q$ in $\mathrm{T}_{S}, \# S \geq \mathrm{c}\left(k_{0}, m, d\right)-q$ is a nonface are supported in some $\mathrm{V}\left(\mathrm{T}_{R}\right), R \subsetneq S$.

(3) Tightness:

there is a collection of $m$ polytopes $Q_{i}$ in $\mathbb{R}^{d}$ with $f_{0}\left(Q_{[m]}\right)=f_{0}\left(P_{[m]}\right)$ for which, for any $-m+1 \leq k \leq d$ with $k+m-1 \leq \frac{d+m-1}{2}$, we have

$$
\begin{aligned}
& \widetilde{h}_{k+m-1}\left(\mathrm{~T}_{[m]}^{\circ}\left(Q_{i}\right)\right)=\widetilde{\nu}\left(f_{0}\left(Q_{[m]}\right), d, k+m-1\right), \text { and } \\
& h_{k+m-1}\left(\mathrm{~T}_{[m]}\left(Q_{i}\right)\right)=\nu\left(f_{0}\left(Q_{[m]}\right), d, k+m-1\right) .
\end{aligned}
$$

Proof. The proof is analogous to Theorem 5.19: The crucial cases to verify are (1b) and (2b). For this, one can disregard deficient subfamilies of $P_{[m]}$ (those with $\xi\left(P_{S}\right)<d$ ), as their contribution is purely combinatorial. For the remaining subfamilies, one can use Lemma 6.10 as in Theorem 5.19.

\section{Mixed faces of Minkowski Sums}

Let $P_{[m]}=\left(P_{1}, \ldots, P_{m}\right)$ be a pure collection of $m$ polytopes in $\mathbb{R}^{d}$ in relatively general position. Every proper face $F \subsetneq\left|P_{[m]}\right|$ has a unique decomposition $F=F_{1}+\cdots+F_{m}$ where $F_{i} \subseteq P_{i}$ is a face. A face $F$ is called mixed if $\operatorname{dim} F_{i}>0$ for all $i=1, \ldots, m$. In this section, we will study the mixed $f$-vector $f^{\operatorname{mix}}\left(P_{[m]}\right)$ giving the number of mixed faces of $\left|P_{[m]}\right|$. Mixed faces and in particular mixed facets are related to the better known mixed cells in mixed subdivisions via liftings; see [dLRS10]. In this section, we prove an upper bound theorem for the number of mixed faces.

Notice, that by definition $f_{-1}^{\text {mix }}\left(P_{[m]}\right)=f_{0}^{\text {mix }}\left(P_{[m]}\right)=0$. Moreover, the 'relatively general position' assumption forces $f_{k}^{\text {mix }}\left(P_{[m]}\right)=0$ for all $k<m$, which also limits the number of summands to $m<d$. One can drop the assumption on general position but this is less natural. Let us start with a simple observation. A face $F \subsetneq\left|P_{[m]}\right|$ is mixed if and only if it is not a face of a subsum in the following sense: For a linear function $\ell$ let us denote by $P_{S}^{\ell}$ the face of $P_{S}$ maximizing $\ell$. Then $F$ is mixed if for all $\ell$ such that $P_{[m]}^{\ell}=F, P_{S}^{\ell} \neq F$ for all $S \subsetneq[m]$. We may now evaluate the mixed faces as the difference of all faces of the relative Cayley complex, minus the non-mixed faces. Following this basic equation gives an inclusion-exclusion, and we obtain that

$$
f_{k}^{\text {mix }}\left(P_{[m]}\right) \leq \sum_{S \subseteq[m]}(-1)^{m-|S|} f_{k}\left(\left|P_{S}\right|\right),
$$


with equality for the number of mixed facets. Let us define the mixed $h$-vector of $P_{[m]}$ by

$$
h_{i+m-1}^{\operatorname{mix}}\left(P_{[m]}\right):=\sum_{S \subseteq[m]}(-1)^{m-|S|} g_{i+|S|-1}^{\langle m-|S|\rangle}\left(\mathrm{T}_{S}^{\circ}\right)
$$

for all $-m+1 \leq i \leq d$. Using (1) and Proposition 5.9 proves the following.

Lemma 7.1. Let $P_{[m]}$ be a collection of polytopes in relatively general position. Then

$$
f_{k}^{\operatorname{mix}}\left(P_{[m]}\right) \leq \sum_{i=-m+1}^{d}\left(\begin{array}{l}
d-i \\
k-i
\end{array}\right) h_{i+m-1}^{\operatorname{mix}}\left(P_{[m]}\right)
$$

for all $k \geq 0$.

Thus, in analogy to the UBT for Minkowski sums, it suffices to prove upper bounds on the mixed $h$-vector of $P_{[m]}$.

Theorem 7.2. Let $P_{[m]}$ be a pure collection of $m$ simplicial polytopes in relatively general position in $\mathbb{R}^{d}$ with $\mathbf{n}=f_{0}\left(P_{[m]}\right)$. Let $\mathrm{T}_{[m]}=\mathrm{T}\left(P_{[m]}\right)$ be the corresponding Cayley complex. Then for $-m+1 \leq k \leq d-m+1$

$$
\begin{aligned}
& h_{k+m-1}^{\operatorname{mix}}\left(P_{[m]}\right) \\
= & \sum_{S \subseteq[m]}(-1)^{m-|S|} g_{i+|S|-1}^{\langle m-|S|\rangle}\left(\mathrm{T}_{S}^{\circ}\right) \\
\leq & \sum_{S \subseteq[m]}(-1)^{m-|S|} \widetilde{\omega}^{\langle m-|S|\rangle}\left(\mathbf{n}_{S}, d, k+|S|-1\right),
\end{aligned}
$$

with equality for some $k_{0}+m-1$ if and only if it holds for all summands.

For mixed facets, this results in the following tight upper bound.

Theorem 7.3. Let $0<m<d$ and $P_{[m]}$ a collection of $m$ simplicial d-polytopes in relatively general position in $\mathbb{R}^{d}$. Then for any Minkowski neighborly family $\mathrm{Nb}_{[m]}$ of d-polytopes with $f_{0}\left(P_{[m]}\right)=f_{0}\left(\mathrm{Nb}_{[m]}\right)$ we have

$$
f_{d-1}^{\operatorname{mix}}\left(P_{[m]}\right) \leq f_{d-1}^{\operatorname{mix}}\left(\mathrm{Nb}_{[m]}\right)
$$

with equality if and only if $P_{[m]}$ is Minkowski neighborly.

This is an immediate consequence of Theorem 7.2.

Proof of Theorem 7.2. We first show the bounds on the initial terms of the mixed $h$-vector, i.e. bounds on $h_{k+m-1}^{\operatorname{mix}}\left(P_{[m]}\right)$ for $k+m-1 \leq \frac{d+m-1}{2}$.

We have

$$
h_{k+m-1}^{\operatorname{mix}}\left(P_{[m]}\right)=\sum_{S \subseteq[m]}(-1)^{m-|S|} g_{k+|S|-1}^{\langle m-|S|\rangle}\left(\mathrm{T}_{S}^{\circ}\right) .
$$

The second sum may be written as

$$
\sum_{S \subseteq[m]} \sum_{\substack{j \in[0, m-|S|] \\
j+m-|S| \text { even }}}\left(\begin{array}{c}
m-|S| \\
j
\end{array}\right)\left(h_{k+|S|-1-j}\left(\mathrm{~T}_{S}^{\circ}\right)-\frac{1}{m-|S|-j+1} \sum_{R \prec S} h_{k+|R|-1-j}\left(\mathrm{~T}_{R}^{\circ}\right)\right)
$$

where we recall that $\prec$ denotes the covering relation. Using Lemma 5.17 we can therefore estimate

$$
\sum_{S \subseteq[m]}(-1)^{m-|S|} g_{k+|S|-1}^{\langle m-|S|\rangle}\left(\mathrm{T}_{S}^{\circ}\right) \leq \sum_{S \subseteq[m]}(-1)^{m-|S|} \widetilde{\omega}^{\langle m-|S|\rangle}\left(f_{0}\left(P_{S}\right), d, k+|S|-1\right) .
$$

The second bound can be derived in a similar manner as the first: Combining the Dehn-Sommerville relations and Lemma 5.14, we rewrite $h_{k+m-1}^{\text {mix }}\left(P_{[m]}\right)=\sum_{S \subseteq[m]}(-1)^{m-|S|} g_{i+|S|-1}^{\langle m-|S|\rangle}\left(\mathrm{T}_{S}^{\circ}\right)$ as a sum of $h_{j}\left(\mathrm{~T}_{S}^{\circ}\right), j \leq \frac{d+|S|-1}{2}$. We can now pair $h$ - and $g$-numbers of $\mathrm{T}_{S}^{\circ}$ and $\mathrm{T}_{R}^{\circ}, R \prec S$ and use Lemma 5.17 to bound each term by the corresponding term of $\widetilde{\omega}$ and $\widetilde{\omega}^{\langle 1\rangle}$. In details:

Recall that as in Lemma 5.14, we may think of the coefficients of $h_{i+m+S}\left(\mathrm{~T}_{S}^{\circ}\right)$ Equation (11) as elements in an array with sides recording $i$ and $|S|$. 
The interplay with the Dehn-Sommerville relations for Cayley complexes now becomes relevant if, in a summand $h_{k+|S|-1-j}\left(\mathrm{~T}_{S}^{\circ}\right)-\frac{1}{m-|S|-j+1} \sum_{R \prec S} h_{k+|R|-1-j}\left(\mathrm{~T}_{R}^{\circ}\right)$ in the Sum (12), $k+|S|-1-j>\frac{d+|S|-1}{2}$. To understand this, we rewrite Sum (12) as

$$
\sum_{j=0}^{m} \sum_{\substack{S \subseteq[m] \\|S|=m-j}} \sum_{R \subseteq S}(-1)^{|S \backslash R|} \frac{1}{|S \backslash R| !} h_{k+|R|-1-j}\left(\mathrm{~T}_{R}^{\circ}\right)
$$

For any $S \subsetneq m$, we can now apply the Dehn-Sommerville relations and Lemma 5.14 to rewrite, for $S \subsetneq[m]$ and $j=m-|S|$,

$$
\begin{aligned}
& \sum_{R \subseteq S}(-1)^{|S \backslash R|} \frac{1}{|S \backslash R| !} h_{k+|R|-1-j}\left(\mathrm{~T}_{R}^{\circ}\right) \\
& =\sum_{\substack{R \subseteq S \\
|S \backslash R| \text { even }}} \frac{1}{|S \backslash R| !} \sum_{T \subseteq R}(-1)^{|T|-|R|} h_{d-k+m-|S|+|T|-|R|}\left(\mathrm{T}_{T}^{\circ}\right) \\
& |R| \geq d-2 k+2 m-2|S|+1
\end{aligned}
$$

and secondly rewrite, for $R \subseteq S$, and $|S \backslash R|$ even, the summands of the identity as

$$
\begin{aligned}
& \sum_{T \subseteq R}(-1)^{|T|-|R|} h_{d-k+m-|S|+|T|-|R|}\left(\mathrm{T}_{T}^{\circ}\right) \\
= & \sum_{\substack{T \subseteq R \\
|R \backslash T| \text { even }}}\left(h_{d-k+m-|S|+|T|-|R|}\left(\mathrm{T}_{T}^{\circ}\right)-\frac{1}{|S|-|T|+1} \sum_{U \prec T} h_{d-k+m-|S|+|U|-|R|}\left(\mathrm{T}_{U}^{\circ}\right)\right) .
\end{aligned}
$$

The claim now follows by application of Lemma 5.17.

\section{REFERENCES}

[Adi15] K. A. Adiprasito, Toric chordality, preprint, arXiv:1503.06640. 22

[AB12] K. A. Adiprasito and B. Benedetti, Subdivisions, shellability, and collapsibility of products, February 2012, to appear in Combinatorica, available at arXiv:1202.6606. 22, 28

[ABG83] K. A. Adiprasito, A. Björner, and A. Goodarzi, Face numbers of Sequentially Cohen-Macaulay complexes and Betti numbers of componentwise linear ideals, preprint, arXiv:1502.01183. 3

[ABPS15] K. A. Adiprasito, P. Brinkmann, A. Padrol, and R. Sanyal, Mixed faces and colorful depth, in preparation, 2015. 2

[BKL86] D. Barnette, P. Kleinschmidt, and C. W. Lee, An upper bound theorem for polytope pairs, Math. Oper. Res. 11 (1986), no. 3, 451-464. 3

[BL80] L. J. Billera and C. W. Lee, Sufficiency of McMullen's conditions for f-vectors of simplicial polytopes., Bull. Am. Math. Soc., New Ser. 2 (1980), 181-185. 11

[BL81] - The numbers of faces of polytope pairs and unbounded polyhedra, European J. of Combinatorics 2 (1981), 307-322. 3

[Bjö80] A. Björner, Shellable and Cohen-Macaulay partially ordered sets, Trans. Amer. Math. Soc. 260 (1980), 159-183. 22

[Bjö03] _ Nerves, fibers and homotopy groups, Journal of Combinatorial Theory, Series A 102 (2003), no. 1, 88 - 93. 16

[Bjö07] _ A comparison theorem for f-vectors of simplicial polytopes, Pure Appl. Math. Q. 3 (2007), no. 1, 347-356. 3

[BWW05] A. Björner, M. L. Wachs, and V. Welker, Poset fiber theorems, Trans. Amer. Math. Soc. 357 (2005), 1877-1899. 16

[Bor48] K. Borsuk, On the imbedding of systems of compacta in simplicial complexes, Fundam. Math. 35 (1948), 217-234. 16

[BM71] H. Bruggesser and P. Mani, Shellable decompositions of cells and spheres, Math. Scand. 29 (1971), 197-205 (1972). 22,28

[BH93] W. Bruns and J. Herzog, Cohen-Macaulay rings, Cambridge Studies in Advanced Mathematics, vol. 39, Cambridge University Press, Cambridge, 1993. 6, 8, 10

[Buc43] R. C. Buck, Partition of space, Amer. Math. Monthly 50 (1943), 541-544. 35, 36

[CD95] R. M. Charney and M. W. Davis, Strict hyperbolization, Topology 34 (1995), no. 2, 329-350. 11

[CLS11] D. A. Cox, J. B. Little, and H. K. Schenck, Toric varieties, Graduate Studies in Mathematics, vol. 124, American Mathematical Society, Providence, RI, 2011. 1

[Dav08] M. W. Davis, The geometry and topology of Coxeter groups, London Mathematical Society Monographs Series, vol. 32, Princeton University Press, Princeton, NJ, 2008. 11

[dLRS10] J. A. de Loera, J. Rambau, and F. Santos, Triangulations, Algorithms and Computation in Mathematics, vol. 25, Springer-Verlag, Berlin, 2010, Structures for algorithms and applications. 25, 26, 39

[Duv96] A. M. Duval, Algebraic shifting and sequentially cohen-macaulay simplicial complexes, Electron. J. Comb. 3 (1996), no. 1 , research paper $\mathrm{r} 21,14.3$ 
[Eis95] D. Eisenbud, Commutative algebra with a view toward algebraic geometry, Graduate Texts in Mathematics, vol. 150, Springer-Verlag, New York, 1995. 6

[EC95] I. Z. Emiris and J. F. Canny, Efficient incremental algorithms for the sparse resultant and the mixed volume, J. Symbolic Comput. 20 (1995), no. 2, 117-149. 2

[FW07] K. Fukuda and C. Weibel, f-vectors of Minkowski additions of convex polytopes, Discrete Comput. Geom. 37 (2007), no. 4, 503-516. 2

[FW10] _ A linear equation for Minkowski sums of polytopes relatively in general position, European J. Combin. 31 (2010), no. 2, 565-573. 2, 23

[FS97] W. Fulton and B. Sturmfels, Intersection theory on toric varieties, Topology 36 (1997), no. 2, 335-353. 2

[Geo08] R. Geoghegan, Topological methods in group theory, Graduate Texts in Mathematics, vol. 243, Springer, New York, 2008. 11

[Grä87] H.-G. Gräbe, Generalized Dehn-Sommerville equations and an upper bound theorem, Beiträge Algebra Geom. (1987), no. $25,47-60.5$

[GS93] P. Gritzmann and B. Sturmfels, Minkowski addition of polytopes: computational complexity and applications to Gröbner bases, SIAM J. Discrete Math. 6 (1993), no. 2, 246-269. 1

[Hib91] T. Hibi, Quotient algebras of Stanley-Reisner rings and local cohomology, Journal of Algebra 140 (1991), no. 2, 336 343. 15

[Hoc77] M. Hochster, Cohen-Macaulay rings, combinatorics, and simplicial complexes, Ring theory, II (Proc. Second Conf., Univ. Oklahoma, Norman, Okla., 1975), Dekker, New York, 1977, pp. 171-223. Lecture Notes in Pure and Appl. Math., Vol. 26. 2, 11

[HR74] M. Hochster and J. L. Roberts, Rings of invariants of reductive groups acting on regular rings are Cohen-Macaulay, Advances in Math. 13 (1974), 115-175. 8

[Hov78] A. G. Hovanskiı̌, Newton polyhedra, and the genus of complete intersections, Funktsional. Anal. i Prilozhen. 12 (1978), no. 1, 51-61. 1

$\left[\mathrm{ILL}^{+} 07\right] \quad$ S. B. Iyengar, G. J. Leuschke, A. Leykin, C. Miller, E. Miller, A. K. Singh, and U. Walther, Twenty-four hours of local cohomology, Graduate Studies in Mathematics, vol. 87, American Mathematical Society, Providence, RI, 2007. 6, 8

[JMR83] W. Julian, R. Mines, and F. Richman, Alexander duality, Pacific J. Math. 106 (1983), no. 1, 115-127. 11

[Kal91] G. Kalai, The diameter of graphs of convex polytopes and $f$-vector theory, Applied geometry and discrete mathematics, DIMACS Ser. Discrete Math. Theoret. Comput. Sci., vol. 4, Amer. Math. Soc., Providence, RI, 1991, pp. 387-411. 19

[KKT15] M. I. Karavelas, C. Konaxis, and E. Tzanaki, The maximum number of faces of the Minkowski sum of three convex polytopes, J. Comput. Geom. 6 (2015), no. 1, 21-74. 2, 23

[KT11] M. I. Karavelas and E. Tzanaki, The maximum number of faces of the Minkowski sum of two convex polytopes, October 2011, preprint, to appear in Discrete Comput. Geom., available at dx.doi.org/10.1007/s00454-015-9726-6. $2,3,22,23,25$

[KT15] _ A geometric approach for the upper bound theorem for Minkowski sums of convex polytopes, 31st International Symposium on Computational Geometry, LIPIcs. Leibniz Int. Proc. Inform., vol. 34, Schloss Dagstuhl. Leibniz-Zent. Inform., Wadern, 2015, pp. 81-95. 28

[Kat12] E. Katz, Tropical intersection theory from toric varieties, Collect. Math. 63 (2012), no. 1, 29-44. 2

[KK79] B. Kind and P. Kleinschmidt, Schälbare Cohen-Macauley-Komplexe und ihre Parametrisierung, Math. Z. 167 (1979), no. 2, 173-179. 22

[Kle64] V. Klee, On the number of vertices of a convex polytope, Canad. J. Math. 16 (1964), 701-720. 2, 5, 10

[Lat91] J.-C. Latombe, Robot motion planning, vol. 25, Kluwer Academic Publishers, Boston, MA, 1991. 1

[MPP11] B. Matschke, J. Pfeifle, and V. Pilaud, Prodsimplicial-neighborly polytopes, Discrete Comput. Geom. 46 (2011), no. 1, 100-131. 2, 12, 23, 36

[McM70] P. McMullen, The maximum numbers of faces of a convex polytope, Mathematika 17 (1970), no. 02, 179-184. 1, 10, 19,22

[McM04] _ Triangulations of simplicial polytopes, Beiträge Algebra Geom. 45 (2004), no. 1, 37-46. 3

[MW71] P. McMullen and D. W. Walkup, A generalized lower-bound conjecture for simplicial polytopes, Mathematika 18 (1971), 264-273. 11

[MNS11] E. Miller, I. Novik, and E. Swartz, Face rings of simplicial complexes with singularities, Math. Ann. 351 (2011), no. 4, 857-875. 2

[MS05] E. Miller and B. Sturmfels, Combinatorial commutative algebra, Graduate Texts in Mathematics, vol. 227, SpringerVerlag, New York, 2005. 4, 6, 7

[Min11] H. Minkowski, Theorie der konvexen körper, insbesondere Begründung ihres Oberflächenbegriffs, Gesammelte Abhandlungen von Hermann Minkowski 2 (1911), 131-229. 1

[Miy89] M. Miyazaki, Characterizations of Buchsbaum complexes, manuscripta mathematica 63 (1989), no. 2, 245-254. 7

[Mot57] T. S. Motzkin, Comonotone curves and polyhedra, Bull. Amer. Math. Soc. 63 (1957), 35.1

[MRTT53] T. S. Motzkin, H. Raiffa, G. L. Thompson, and R. M. Thrall, The double description method, Contributions to the theory of games, vol. 2, Annals of Mathematics Studies, no. 28, Princeton University Press, Princeton, N. J., 1953, pp. $51-73.1$

[Nov03] I. Novik, Remarks on the upper bound theorem, J. Combin. Theory Ser. A 104 (2003), 201-206. 2

[Nov05] _ _ On face numbers of manifolds with symmetry, Adv. Math. 192 (2005), no. 1, 183-208. 2

[NS09] I. Novik and E. Swartz, Applications of Klee's Dehn-Sommerville relations, Discrete Comput. Geom. 42 (2009), no. $2,261-276.3,5$ 
[NS12] _ Face numbers of pseudomanifolds with isolated singularities, Math. Scand. 110 (2012), no. 2, 198-222. 2

[PS93] P. Pedersen and B. Sturmfels, Product formulas for resultants and Chow forms, Math. Z. 214 (1993), no. 3, 377-396. 2

[Rei76] G. A. Reisner, Cohen-Macaulay quotients of polynomial rings, Advances in Math. 21 (1976), no. 1, 30-49. 2, 7

[RS72] C. P. Rourke and B. J. Sanderson, Introduction to Piecewise-Linear Topology, Springer, New York, 1972, Ergebnisse Series vol. 69. 11

[San09] R. Sanyal, Topological obstructions for vertex numbers of Minkowski sums, J. Combin. Theory Ser. A 116 (2009), no. 1, 168-179. 2

[Sch81] P. Schenzel, On the number of faces of simplicial complexes and the purity of Frobenius, Math. Z. 178 (1981), no. 1, 125-142. 2, 7, 8, 9, 15

[Sch82] _ Dualisierende Komplexe in der lokalen Algebra und Buchsbaum-Ringe, Lecture Notes in Mathematics, vol. 907, Springer-Verlag, Berlin, 1982, With an English summary. 7, 8, 9, 15

[Sch93] R. Schneider, Convex bodies: the Brunn-Minkowski theory, Encyclopedia of Mathematics and its Applications, vol. 44, Cambridge University Press, Cambridge, 1993. 1

[Sta75] R. P. Stanley, The upper bound conjecture and Cohen-Macaulay rings, Studies in Appl. Math. 54 (1975), no. 2, 135-142. 2, 10

[Sta87] - Generalized H-vectors, intersection cohomology of toric varieties, and related results, Commutative algebra and combinatorics (Kyoto, 1985), Adv. Stud. Pure Math., vol. 11, North-Holland, Amsterdam, 1987, pp. 187-213. 22

[Sta93] _ A monotonicity property of h-vectors and $h^{*}$-vectors, European J. Combin. 14 (1993), no. 3, 251-258. 19

[Sta96] _ Combinatorics and commutative algebra, second ed., Progress in Mathematics, vol. 41, Birkhäuser Boston Inc., Boston, MA, 1996. 4, 6, 7, 21, 22

[ST10] R. Steffens and T. Theobald, Combinatorics and genus of tropical intersections and Ehrhart theory, SIAM J. Discrete Math. 24 (2010), no. 1, 17-32. 2

[Ste26] J. Steiner, Einige Gesetze über die Theilung der Ebene und des Raumes, Journal für die reine und angewandte Mathematik 1 (1826), 349-364. 35

[Stu02] B. Sturmfels, Solving systems of polynomial equations, CBMS Regional Conference Series in Mathematics, vol. 97, Published for the Conference Board of the Mathematical Sciences, Washington, DC, 2002. 1

[Swa05] E. Swartz, Lower bounds for $h$-vectors of $k$-CM, independence, and broken circuit complexes., SIAM J. Discrete Math. 18 (2005), no. 3, 647-661. 19

[Wei12] C. Weibel, Maximal f-vectors of Minkowski sums of large numbers of polytopes, Discrete Comput. Geom. 47 (2012), no. 3, 519-537. 2

[Zee66] E. C. Zeeman, Seminar on combinatorial topology, Institut des Hautes Etudes Scientifiques, Paris, 1966.11

[Zie95] G. M. Ziegler, Lectures on Polytopes, Graduate Texts in Mathematics, vol. 152, Springer, New York, 1995, Revised edition, 1998; seventh updated printing 2007. 1, 10, 22, 25

Einstein Institute for Mathematics, Hebrew University of Jerusalem, Jerusalem, Israel

Email address: adiprasito@math.huji.ac.il

Fachbereich Mathematik und Informatik, Freie Universität Berlin, Berlin, Germany

Email address: sanyal@math.fu-berlin.de 